Aus der Klinik für Klinische Neurophysiologie

(Prof. Dr. med. W. Paulus)

der Medizinischen Fakultät der Universität Göttingen

\title{
Long-term memory encoding of facial information in humans: an EEG and tACS study
}

\author{
INAUGURAL-DISSERTATION \\ zur Erlangung des Doktorgrades \\ der Medizinischen Fakultät der \\ Georg-August-Universität zu Göttingen
}

\author{
vorgelegt von \\ Sibel Veit \\ aus \\ Detmold
}

Göttingen 2020 
Dekan:

\section{Betreuungsausschuss}

Betreuer:

Ko-Betreuerin:

\section{Prüfungskommission}

Referent:

Ko-Referentin:

Drittreferent/in:

Datum der mündlichen Prüfung:
Prof. Dr. med. W. Brück

Prof. Dr. med. W. Paulus

PD Dr. rer. nat. K. Jordan

PD Dr. rer. nat. K. Jordan

08.09 .2021 
Hiermit erkläre ich, die Dissertation mit dem Titel "Long-term memory encoding of facial information: an EEG and tACS study" eigenständig angefertigt und keine anderen als die von mir angegebenen Quellen und Hilfsmittel verwendet zu haben.

Göttingen, den 
Die Daten, auf denen die vorliegende Arbeit basiert, wurden teilweise publiziert:

Alekseichuk I, Turi Z, Veit S, Paulus W (2019): Model-driven neuromodulation of the right posterior region promotes encoding of long-term memories. Brain Stimulation 13(2), 474483 


\section{Table of contents}

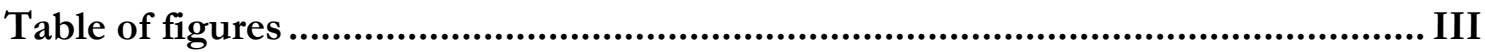

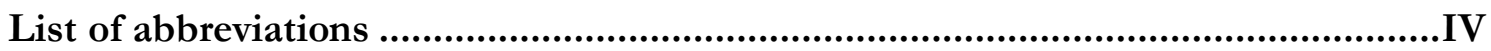

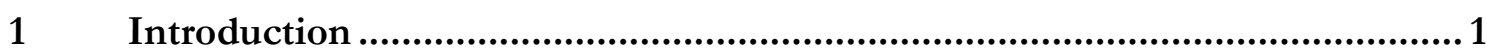

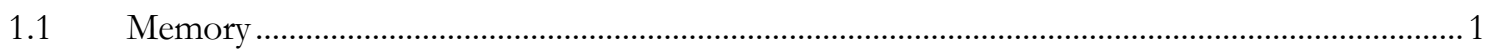

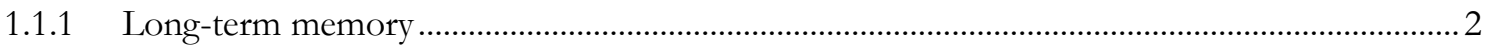

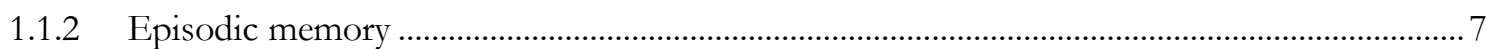

1.1.3 Anatomical structures of the episodic memory .......................................................................

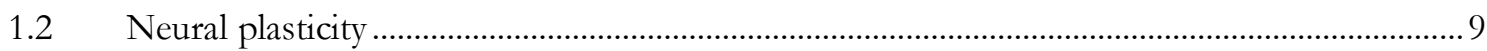

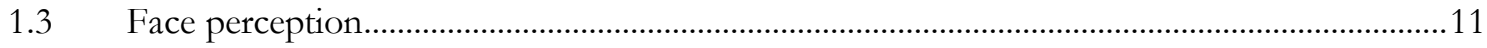

1.4 Temporo-parieto-occipital area......................................................................................... 13

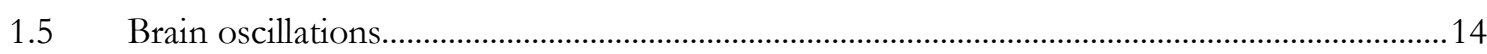

1.6 Neuroimaging - electroencephalography (EEG) ................................................................... 18

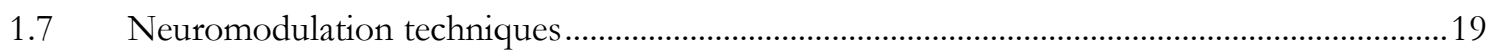

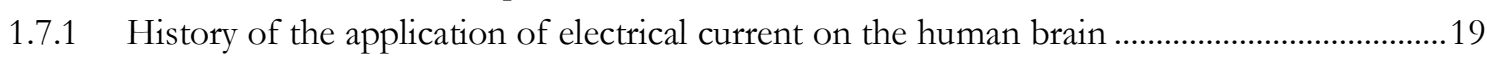

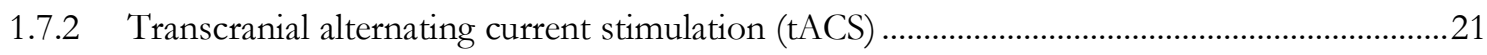

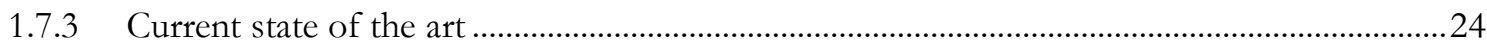

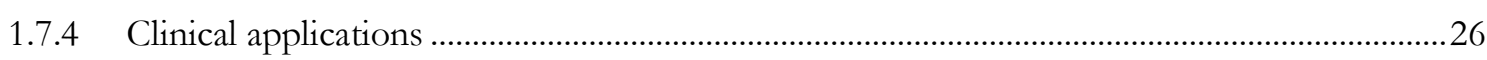

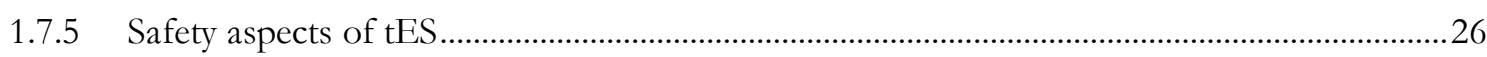

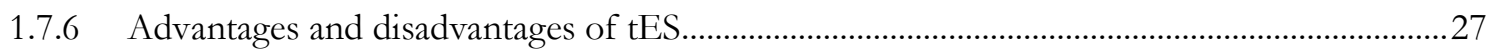

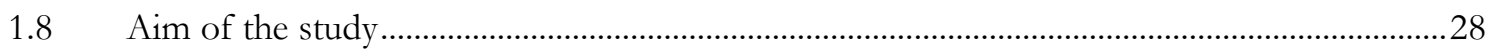

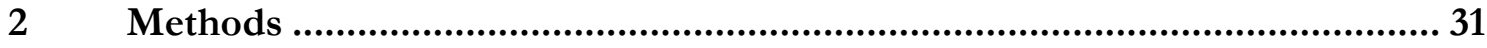

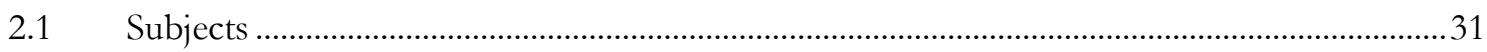

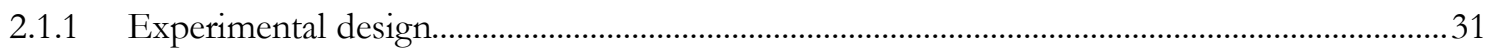

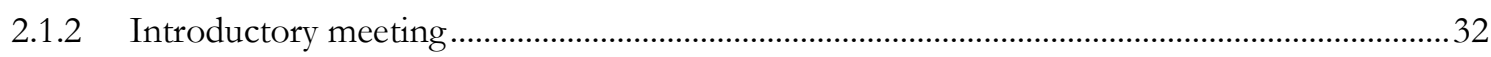

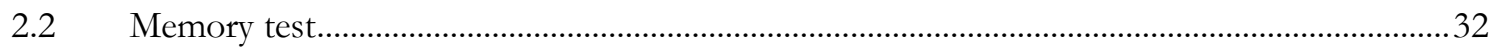

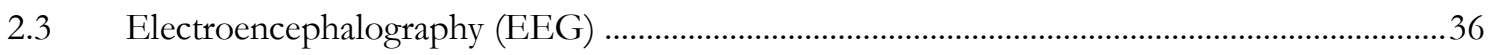

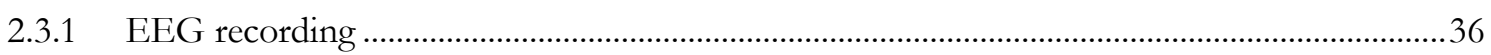

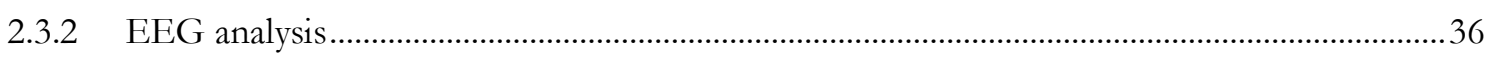

2.4 Transcranial alternating current stimulation (tACS) ...................................................................

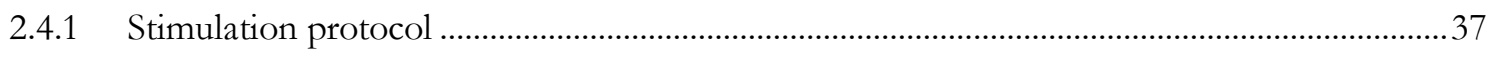

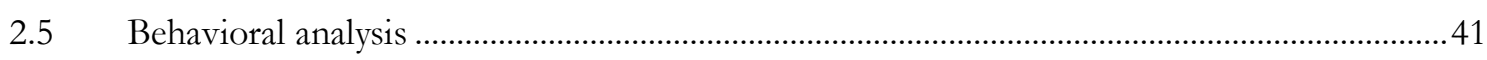

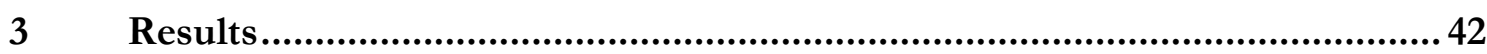

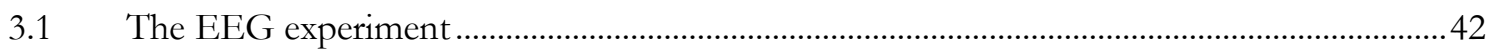

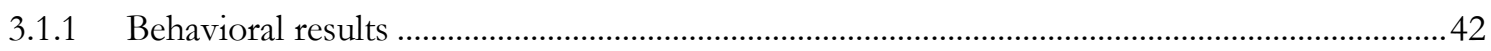

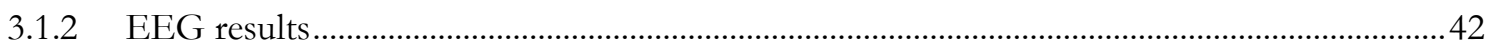

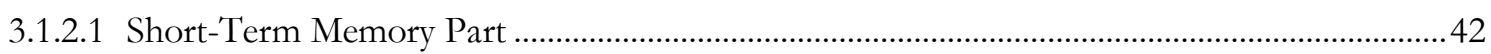




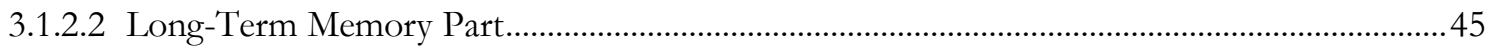

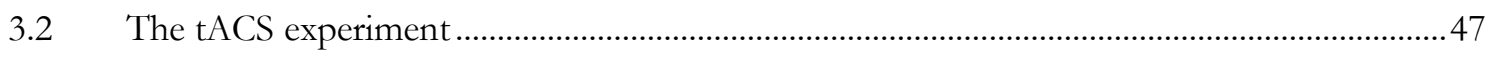

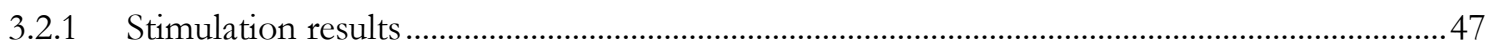

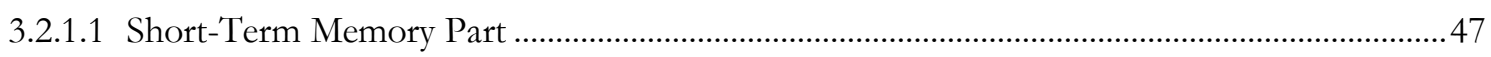

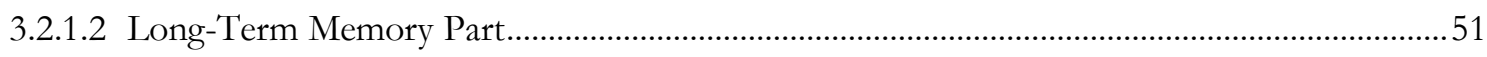

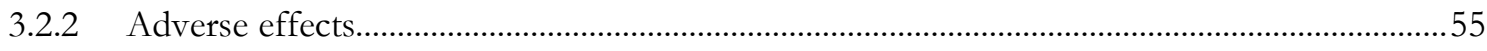

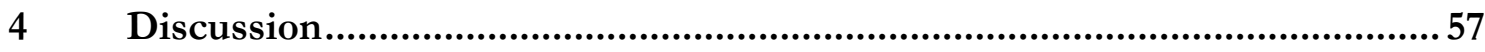

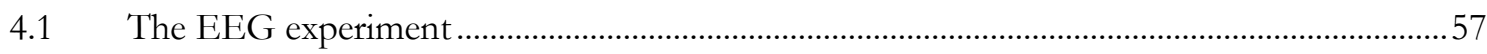

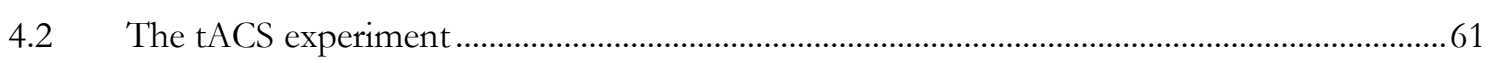

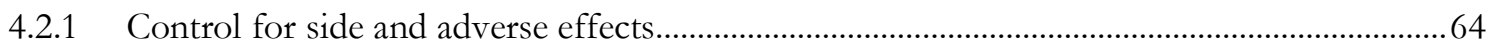

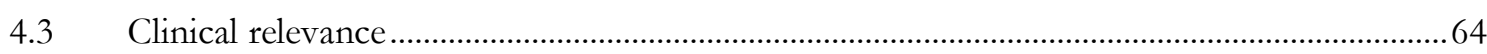

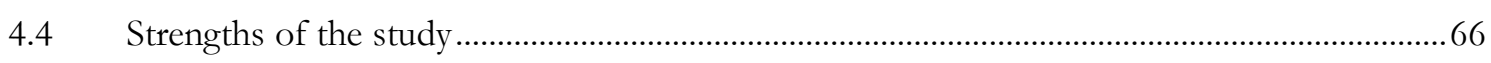

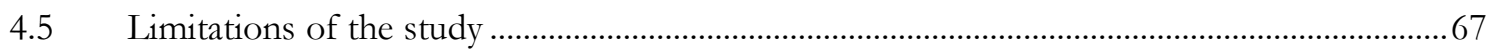

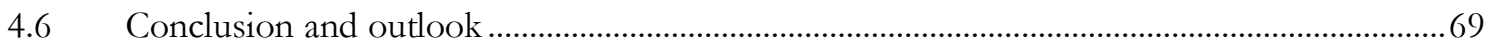

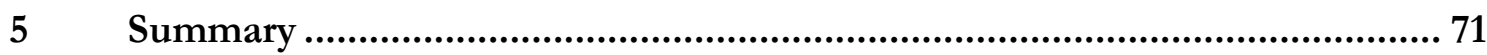

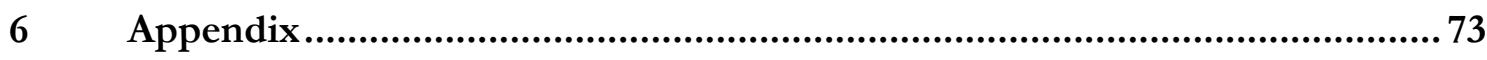

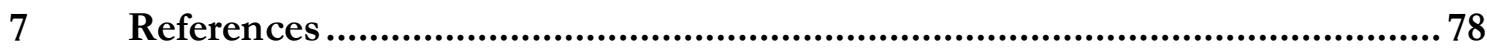




\section{Table of figures}

Figure 1: Multistore model according to Atkinson and Shiffrin............................................................... 3

Figure 2: Levels-of-processing model according to Craik and Lockhart............................................... 4

Figure 3: Long-term memory model with procedural-declarative distinction and underlying anatomic brain regions according to Squire and Zola................................................................... 5

Figure 4: Processing-based memory model according to Henke. …….................................................6

Figure 5: Schematic anatomical architecture of the brain (cross section) ..............................................12

Figure 6: Transcranial alternating current oscillates with a sinusoidal wave form.................................22

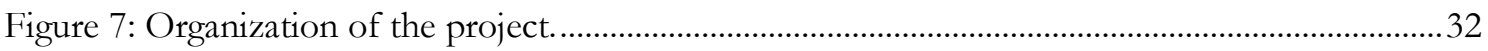

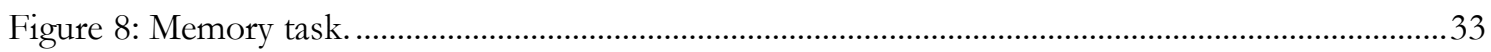

Figure 9: Performance in the first pilot experiment during the Short-Term Memory Retrieval Part as the function of the span..

Figure 10: Performance in the second pilot experiment during the Long-Term Memory

Retrieval Part as the function of the time delay between Encoding and LTM

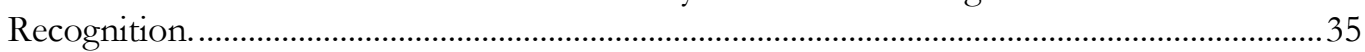

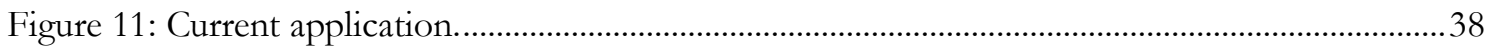

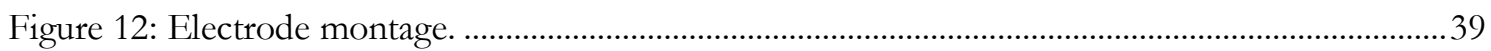

Figure 13: Simulation of the electric field during the stimulation of the left TPO area.......................40

Figure 14: Simulation of the electric field during the stimulation of the right TPO area.....................40

Figure 15: Topographic map of the human brain during memory encoding for subsequently forgotten items in the Short-Term Memory Task......................................................................4

Figure 16: Topographic map of the human brain during memory encoding for subsequently remembered items in the Short-Term Memory Task. .

Figure 17: Difference in brain activity during encoding between subsequently remembered and forgotten answers in the Short-Term Memory Task.

Figure 18: Topographic map of the human brain during memory encoding for subsequently forgotten items in the Long-Term Memory Task.

Figure 19: Topographic map of the human brain during memory encoding for subsequently remembered items in the Long-Term Memory Task...

Figure 20: Difference in brain activity during encoding between subsequently remembered and forgotten answers in the Long-Term Memory Task.

Figure 21: Placebo normalized changes of the mean performance during the Short-Term Memory Part..

Figure 22: Mean memory performance in the Short-Term Memory Part..

Figure 23: Placebo normalized changes of the mean reaction time during the Short-Term Memory Part.

Figure 24: Mean reaction time in the Short-Term Memory Part.......................................................... 51

Figure 25: Mean confidence levels during the Long-Term Memory Part............................................... 52

Figure 26: Mean memory performance in the Long-Term Memory Part.............................................53

Figure 27: Weighted performance during the Long-Term Memory Recognition...................................54

Figure 28: The individual performance (in \%) in the Long-Term Memory Part during tACS over the right TPO area relative to the sham stimulation..............................................................5

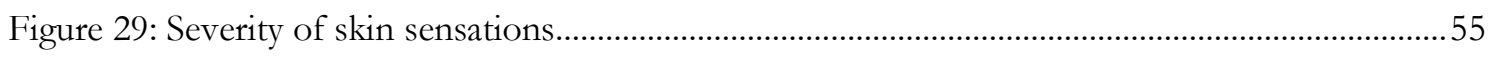

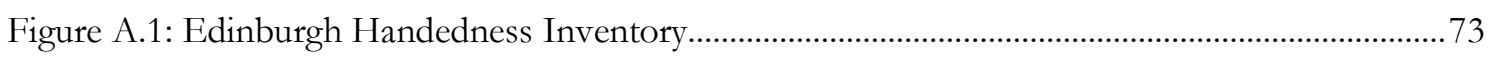

Figure A.2: Questionnaire on exclusion criteria for non-invasive brain stimulation and contents of the medical history and physical examination... 


\section{List of abbreviations}

AE

BMI

DBS

DLPFC

EEG

ERD

ERS

FFA

fMRI

LFP

LH

LTD

LTM

LTP

MTL

NIBS

OFA

PFC

$\mathrm{RH}$

STDP

STM

STS

tACS

tDCS

tES

TMS

TPO

tRNS
Adverse Effect

Body Mass Index

Deep brain stimulation

Dorsolateral prefrontal cortex

Electroencephalography

Event-related desynchronization

Event-related synchronization

Fusiform Face Area

Functional magnetic resonance imaging

Local field potential

Left hemisphere

Long-term depression

Long-term memory

Long-term potentiation

Medial temporal lobe

Non-invasive brain stimulation

Occipital Face Area

Prefrontal cortex

Right hemisphere

Short-term dependent plasticity

Short-term memory

Superior temporal sulcus

Transcranial alternating current stimulation

Transcranial direct current stimulation

Transcranial electric current stimulation

Transcranial magnetic stimulation

Temporo-parieto-occipital

Transcranial random noise stimulation 


\section{Introduction}

Human faces are the fundamental key to social interaction (Martinez 2017; Zhao et al. 2018). Thus, one of the most essential properties of the human brain for interindividual communication is its ability for face perception, memory, and recognition. Impaired face recognition is a severe limitation and prevents concerned people from normal participation in society even up to placing them in social isolation.

Only recently, transcranial alternating current stimulation (tACS) as a form of non-invasive brain stimulation (NIBS) has gained momentum for entraining oscillatory brain activity to induce neuroplasticity (Antal et al. 2008; Ozen et al. 2010; Antal and Paulus 2013; Yavari et al. 2018). This enables the exploration of functional brain changes underlying memory formation. If implemented right, tACS can be a highly suitable tool for enhancing face memory encoding processes, and eventually evolve into a valuable therapeutic approach for patients suffering from prosopamnesia and prosopagnosia. This thesis will at first focus on the still debated mechanisms of facial encoding into episodic long-term memory. It will be considered the underlying oscillatory activity in the neocortex during the memory encoding as shown with high-density electroencephalography. Then, this thesis will discuss how and to what extent transcranial alternating current stimulation can leverage the electroencephalography results to affect brain oscillations crucial for the encoding and enhance subsequent facial recognition.

\subsection{Memory}

Memory is commonly defined as the persistence of brain states over time (Chaudhuri and Fiete 2016). It ascribes changes in brain connections and neural activity enduring the internal and external stimuli that induced these neural states. However, memory's stability does not necessarily mean resistance to changes that occur throughout the entire lifetime (Moscovitch et al. 2016). A theory of memory traces by Richard Semon describes memorization or learning as the activation of little neuronal cell groups resulting in persistent modifications so that specific cues can reactivate them for retrieval (Poo et al. 2016). A criterion for dividing memory into short-term memory (STM; or working memory) and long-term memory (LTM) is the distinction in persistence times of memories (Chaudhuri and Fiete 2016). While STM is believed to have limited capacity about tens of seconds due to continuous activity, LTM can last for decades. This permanence is attributed to morphological chances as e.g. in 
synaptic weights and neuronal structure. Limiting factors for memory are noise perturbing correct storage, decay, so that memory is lost due to its instability, and interference by other memories that could interact and at worst eliminate each other.

The creation of memories is very complex, and its development requires distinct formative steps (Walker and Stickgold 2004). These developmental stages are encoding, consolidation, recall or retrieval, and reconsolidation. First, encoding describes the creation of a new memory by rebuilding internal representations of perceived stimuli. Then, memory is consolidated by transforming internal representations into stable brain states becoming resistant to interference (Walker and Stickgold 2004; Dudai et al. 2015). Consolidation occurs at a synaptic and systemic level (Dudai et al. 2015). While at the level of synapses memory is transformed into an enduring form at the cellular basis in a neural circuit, system consolidation comprises the rearrangement of impressions over ensembles of brain circuits. When the encoded information corresponds existing knowledge, the mental schema helps to induce rapid system consolidation by the upregulation of specific neural links. However, consolidation does not only rely on the stabilization of memories but also refers to the process of enhancement or reconsolidation arising during sleep (Buzsáki 1989; Walker und Stickgold 2004). It has been assumed for a long time that consolidation is only completed when two processing levels are passed (Geiger et al. 2016). At first, consolidation is believed to occur in synaptic modifications lasting for several hours. Then, the second level could exclusively be achieved during sleep as a condition for more complex system reorganization. This concept has recently been challenged by some studies indicating that consolidation is started directly and independently from sleep. Consolidated memory can then be retrieved by recall or recognition (Walker and Stickgold 2004; Antony et al. 2017). During recognition the presented stimulus is compared with existing knowledge and is then associated to it. In contrast, recall makes reconstruction mandatory. With retrieval memory is returned to be fragile as well as active and needs to be reconsolidated (Schwabe et al. 2014). Accordingly, retrieval enables memory to be integrated into preexisting knowledge and facilitates accessibility (Antony et al. 2017). Finally, reconsolidation allows the modification and even the impairment of already consolidated information and results in a stable condition again (Schwabe et al. 2014).

\subsubsection{Long-term memory}

In contrast to the time- and space-limited STM, that is only able to provide limited capacity for actively holding information, LTM is believed to have a theoretically limitless store capacity over time (Cowan 2008). Its durability is based on translational and transcriptional 
processes resulting in biochemical and morphological modifications in the course of neural plasticity (see Chapter 1.2) (Kukushkin and Carew 2017). To guarantee persistence independent of the lifespan of proteins and cells, brain states are very likely stabilized by circuitry (Chaudhuri and Fiete 2016). Positive feedback enables these brain states to endure the exciting stimulus and the ability of molecules to transfer their status to copies. For instance, the active form of cytoplasmatic polyadenylation element binding protein, that is responsible for protein translation in synapses, was found to be causal for memory persistence and learning (Kandel et al. 2014).

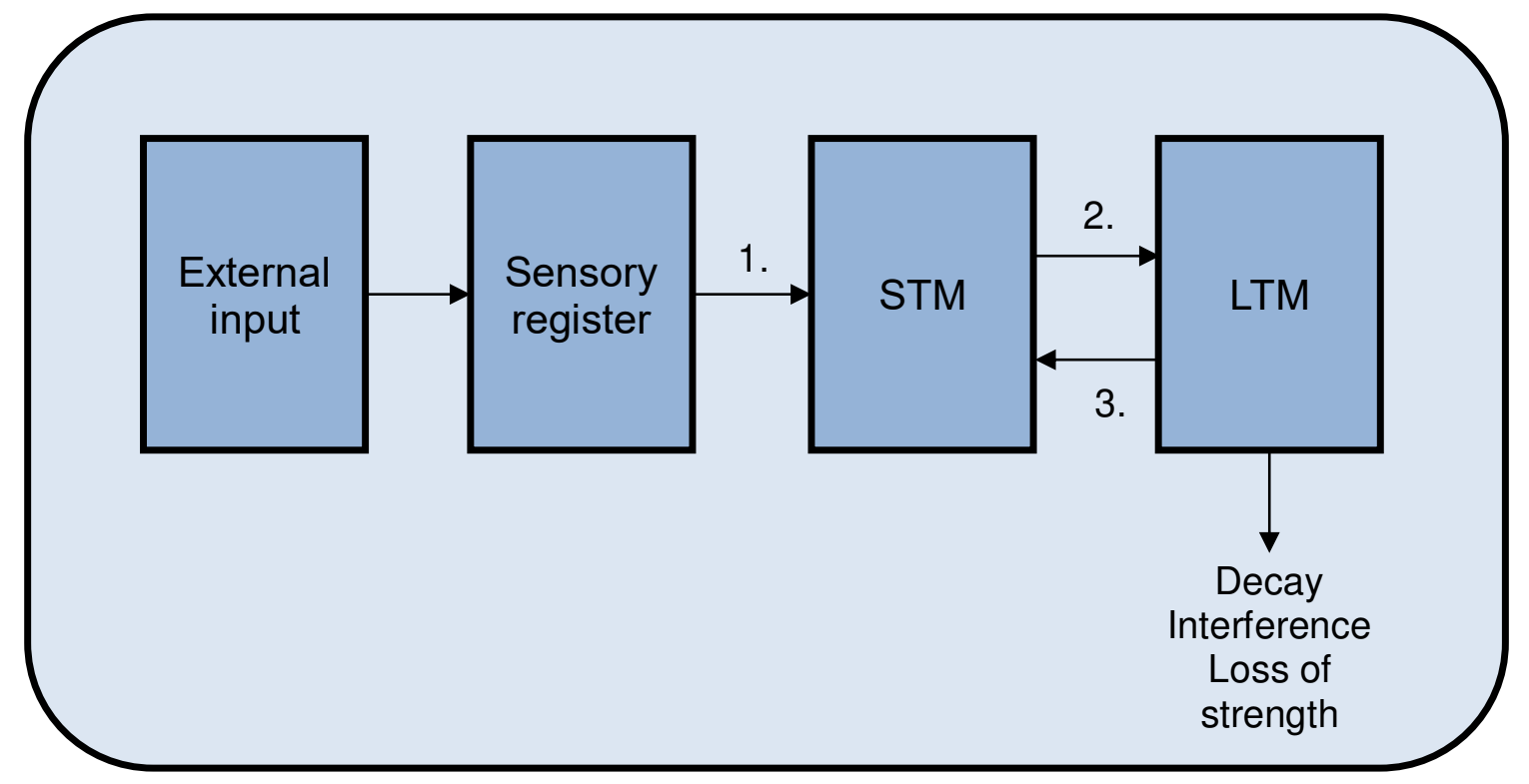

Figure 1: Multistore model according to Atkinson and Shiffrin. 1. = attention; 2. = transfer; 3. = retrieval. (Own illustration based on Atkinson and Shiffrin 1968, modified.)

In the last century, different LTM models have been formulated. In 1968, Atkinson and Shiffrin published their concept of the multistore model of the human memory, that is divided into three components: sensory memory, STM and LTM (see Figure 1) (Atkinson and Shiffrin 1968). The sensory memory captures all perceptible input and is divided into sub-categories aligned with the different senses. When attention is paid on a sensory stimulus, information can enter the STM. However, information will fade due to displacement or decay if it is not actively maintained. From STM information can be transferred into LTM. Even here, memory can become inaccessible by interference, decay, or by failure during retrieval. Retrieval is necessary to remember stimuli while information is relocated back to STM to become available. 
In 1972, Tulving established a more detailed concept of the composition of LTM (Tulving 1986). While procedural memory includes unconscious knowledge comprising motor skills like bicycling that can be performed automatically, semantic and episodic memory contain conscious information and general facts. Accordingly, common knowledge is stored in the semantic store, whereas the episodic component contains information about personal events and biographic experiences.

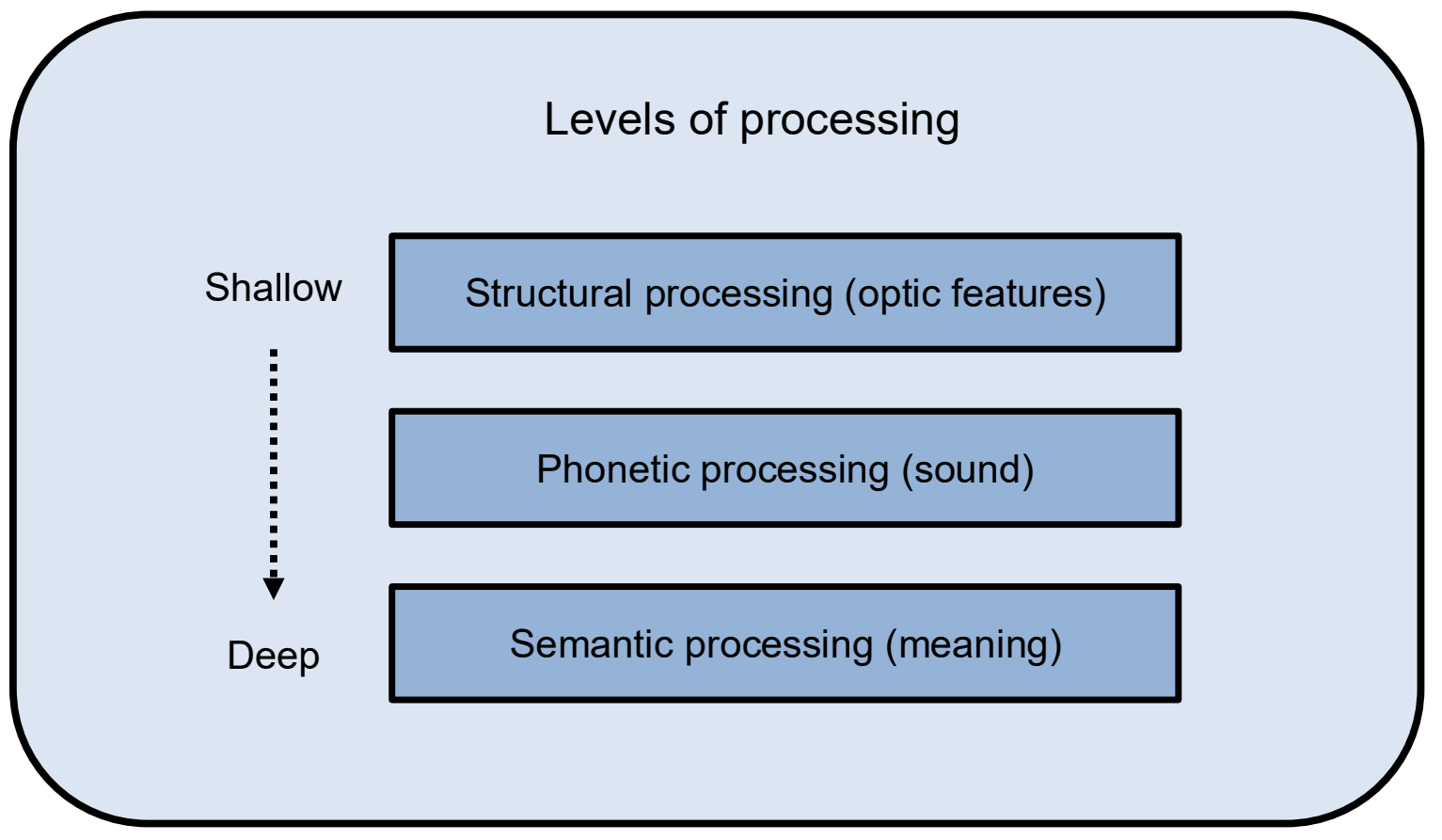

Figure 2: Levels-of-processing model according to Craik and Lockhart. (Own illustration based on Craik and Lockhart 1972, modified.)

In contrast to these linearly structured models, Craik and Lockhart postulated the levels-ofprocessing approach in 1972 (see Figure 2) (Craik and Lockhart 1972). This model considers how and how deeply information is processed with regard to time, stimulus intensity, and mental exertion during processing as the causal factors for memory robustness. Consequently, deeper processed information will be stored longer and more resistant to interference. 


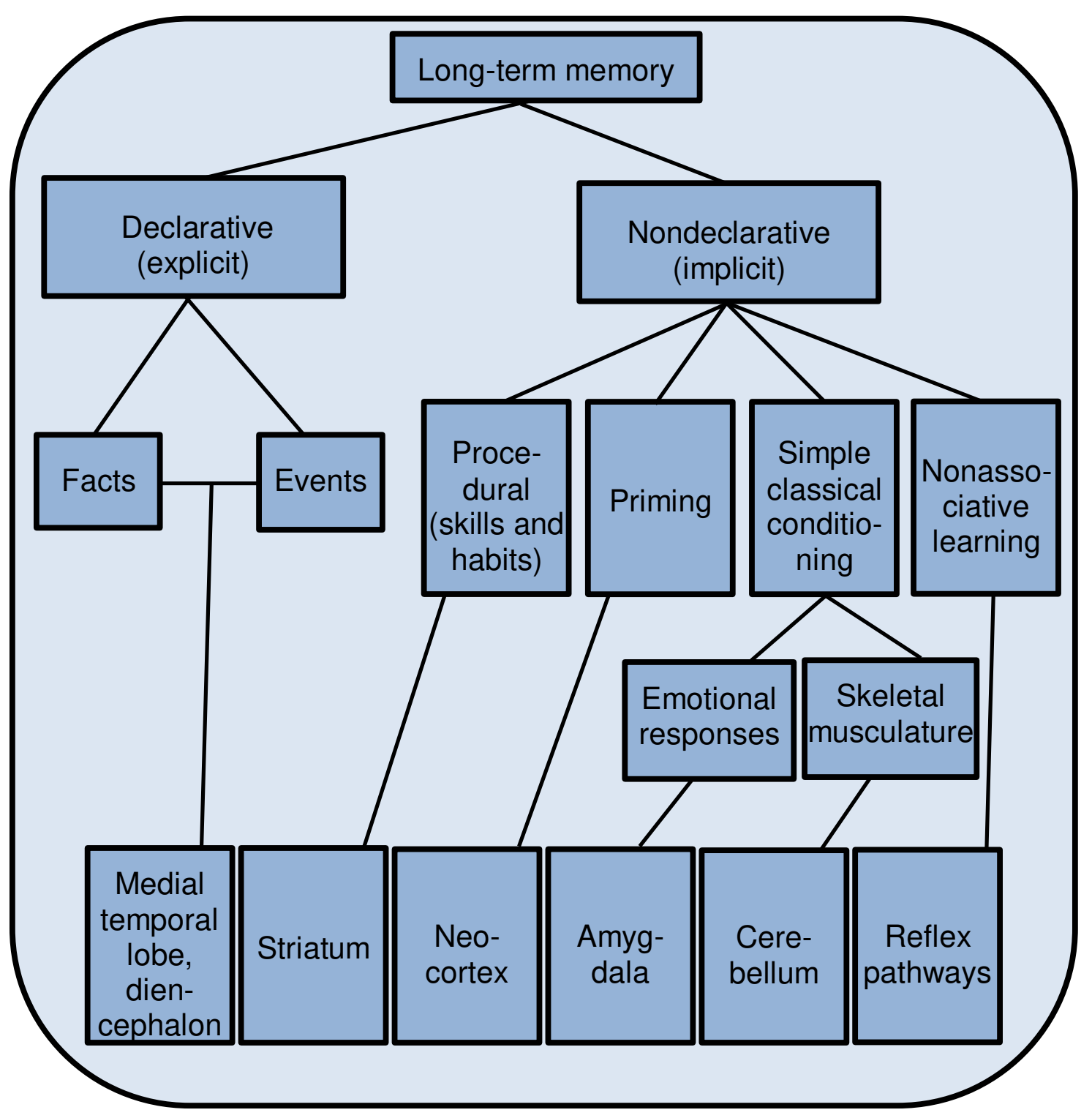

Figure 3: Long-term memory model with procedural-declarative distinction and underlying anatomic brain regions according to Squire and Zola. (Own illustration based on Squire and Zola 1996, modified.)

In 1980, Cohen and Squire extended the idea of a linearly structured memory model by adding a distinction between declarative and non-declarative memory (Cohen and Squire 1980). In the following years this two-class model, dividing memory processes dichotomously into conscious (declarative) and unconscious (non-declarative) operations, was validated and developed until the substantial study by Squire and Zola in 1996 (see Figure 3). According to that, non-declarative information (also referred to as implicit memory) involves procedural memory (including motor skills, motor learning and emotional processes), priming, non-associative learning and classical conditioning (Squire and Zola 1996). In contrast the declarative component (also referred to as explicit memory) is divided into semantic and episodic memory. Moreover, the model reports the anatomic regions that 
represent the different memory entities: the declarative memory is dependent of the medial temporal lobe (MTL) and the diencephalon, in contrast the non-declarative memory requires various brain areas (procedural memory: striatum; priming: neocortex; classical conditioning: amygdala and cerebellum; non-associative learning: reflex pathways).

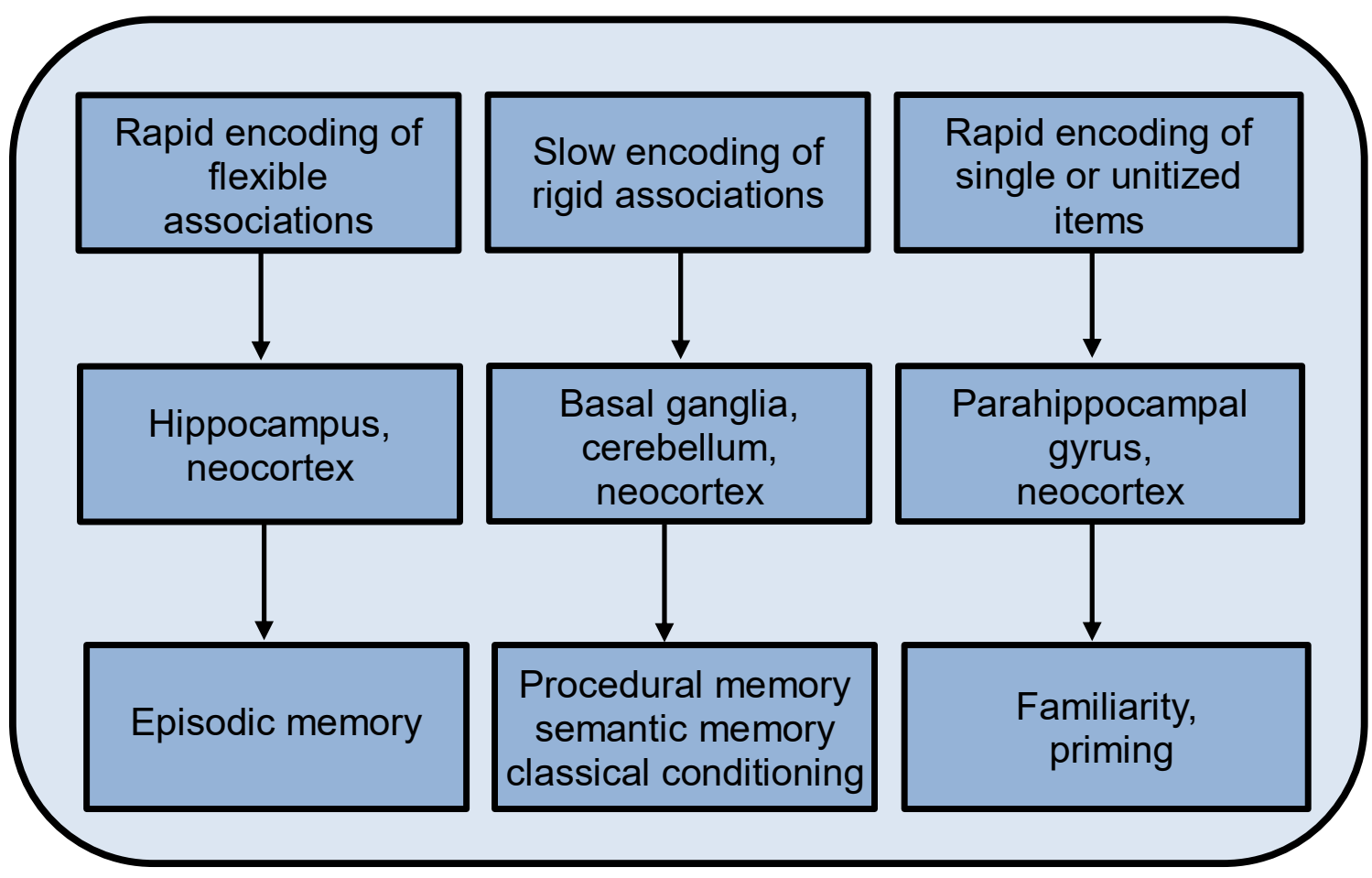

Figure 4: Processing-based memory model according to Henke. (Own illustration based on Henke 2010, modified.)

In the last decade, the two-class model has created numerous controversies and raised alternative models. For example, a corner-stone study by Henke in 2010 conceptualizes a model based on memory processing and thereby independent of consciousness (see Figure 4) (Henke 2010). The model suggests that only the fast encoding and the flexibility of memory associations of the episodic memory are reliant on the hippocampus and the neocortex. Encoding of stimuli based on priming or familiarity are hypothesized to be quickly stored in dependence of the parahippocampal gyrus and the neocortex. In contrast, the semantic and procedural memory as well as classical conditioning involve slow and inflexible storage of memory and are encoded relying on the basal ganglia, cerebellum and neocortex. 


\subsubsection{Episodic memory}

The previous section also focused on the differentiation of memory types by temporal duration and anatomic correlate; nevertheless, a substantial classification is made by the content stored in various parts of the memory. When personal events and autobiographic facts are memorized, information is integrated into episodic memory with a specific representation of the time and place, where the information was acquired (Moscovitch et al. 2016). Tulving first described the concept of this notable cognitive system in contrast to semantic memory in 1972 and declared it as unique in his autonoetic character as it is the only store in the human brain connecting memories to personal remembrance and feelings (Tulving 1986). According to Cohen and Squire's memory model, it is part of the declarative memory that contains LTM about conscious events (Cohen and Squire 1980). It is supposed that episodic memory is a recent evolutionary step in the history of mankind that might have grown out of semantic memory and is even in children not fully developed until the age of four indicating that episodic memory is not fundamentally essential for survival (Tulving 2002). However, there is still a debate whether episodic memory exists across species or if it is a special feature of the human brain (Allen and Fortin 2013). Even if episodic memory is broadly accepted today as an existing sub-unit of LTM, investigation on this topic gained currency only in recent time (Mahr and Csibra 2017). As the episodic memory saves spatialtemporal information, it leads to the reexperience of the event (Tulving 2002; Mahr and Csibra 2017). Lesions in the representative anatomical structures do not only affect the recollection of episodes but additionally cause the loss of future planning indicating that experiences are causal for the ability to imagine forth-coming incidents (Mahr and Csibra 2017). Patients are not able to speculate about possible scenarios or to legitimize their behavior in the past. Hence, they are strongly impaired in social interactions and communication leading to social exclusion.

\subsubsection{Anatomical structures of the episodic memory}

Several neuroimaging studies have documented the particular role of the MTL for episodic memory and for the encoding of specific events into complex associations as well as for retrieving them (Rugg and Vilberg 2013; Dudai et al. 2015; Ison et al. 2015; Eichenbaum 2017). Previous research has established the causal importance of the hippocampus in binding new memories into episodic representations (Tulving 2002; Dragoi and Buzsáki 2006; Ison et al. 2015; Poo et al. 2016). Recent investigation in rodents suggests that the dorsal hippocampus participates in creating locational representations, while ventral neurons are involved in categorizing experiences in various contexts (Eichenbaum 2017). According 
to Buzsáki, it also operates as a navigator in the recollection of memory (Buzsáki 2013). The dentate gyrus, a part of the hippocampus, is responsible for communicating with other brain areas, for example, it serves as an entrance to input from the entorhinal cortex and projects to various brain areas and interneurons (Poo et al. 2016). Encircling structures like the entorhinal, perirhinal and parahippocampal areas are also involved in episodic memory (Rugg and Vilberg 2013). Some authors believe that the parahippocampal cortex offers information about the context, while the perirhinal cortex provides object information (Eichenbaum 2017). The hippocampus is then binding information from both sub-systems into contextual episodes. Projections from the perirhinal and parahippocampal cortex go to the entorhinal cortex, which in turn projects to the hippocampus that consequently integrates all information (Moscovitch et al. 2016).

Some studies have proven interconnectivity between prefrontal areas and the hippocampus (Barbas 2013; Eichenbaum 2017). The prefrontal cortex (PFC) is connected to executive functions, e.g. attention and decision making, and to working memory (Alekseichuk et al. 2016; Girotti et al. 2018). It exercises control via top-down-processes and stores rather abstract information about episodes (Eichenbaum 2017). Impairment does not directly affect episodic memory, but it deteriorates remembrance with distraction and leads to disturbance by irrelevant information. Several disconnection studies showed that bilateral damages as well as inactivation of each of both regions in one hemisphere lead to deficits in episodic memory, while ipsilateral lesions leaving the other hemisphere unattached result in normal episodic memory. This indicates a crucial role of the collaboration of the PFC and hippocampus in one hemisphere.

A famous and well-studied example for the causal role of the MTL in episodic memory was the patient H.M., who suffered from untreatable epilepsy (Allen and Fortin 2013; Pfaff 2013; Korte and Schmitz 2016). After a bilateral medial lobectomy his epilepsy was cured, but instead he came down with a serious anterograde and retrograde amnesia. He could not remember the last two years before surgery; however, earlier episodes were unaffected. Additionally, working memory and semantic memory were intact. Moreover, H.M. was not able to form new episodic LTM from the timepoint of the surgery. This pattern of disability gives evidence for the fact that the MTL is highly important for the creation and retrieval of episodic memory. However, this case raised the question to what extent the MTL in general and the hippocampus in particular are responsible for the storage of LTM. There is still a debate on whether episodic LTM always relies on the hippocampus or whether it might become independent of it after time. Based on recent experiments, some authors believe that episodic memory does not always stay in the hippocampus but can also be transferred to 
neocortex (Moscovitch et al. 2016). Evidence comes from amnestic patients whose remote memories are unaffected but recent events are impaired indicating that the process of memory reorganization storage might rely on neocortical structures (Squire et al. 2015). Consequently, memory is first retained in the hippocampus and the neocortex and then by system consolidation rearranged in neocortical areas into prior knowledge. Finally, memory might be independent of hippocampal representations.

\subsection{Neural plasticity}

The capability of the neural circuitry to adjust to internal or external changes for the whole lifetime is often referred to as brain plasticity (Pascual-Leone et al. 2005; Ganguly and Poo 2013). Plasticity is highly important for brain functioning not only for adapting to changing circumstances and memory formation but also for regeneration after brain injury (Antal et al. 2008). The adaption of neural circuits then leads to a modification of behavior, thoughts, and morphological changes of brain structures (Pascual-Leone et al. 2005; Ganguly and Poo 2013). These modifications are regularly occurring activity dependent. As the human brain is limited in space and power, neuroplasticity is an obligatory consequence to increase efficacy (Pascual-Leone et al. 2005). In 1949, Donald Hebb introduced the idea of that learning relies on synaptic connections and their inherent strength (Brown et al. 2009; Poo et al. 2016). He raised the concept of two neurons being connected by an excitatory synapse when neuron $\mathrm{A}$ is repetitively activating neuron $\mathrm{B}$ which will likely lead to higher interactions between the neurons. Thus, the connection will get reinforced. In sum, Hebb's concept highlights the importance of locality, time of firing, and interactivity between neurons.

Synaptic plasticity refers to alterations of synaptic transmission (Citri and Malenka 2008). The modifications at the level of synapses affecting the synapse efficacy and strength could play a causal role in the encoding of STM and LTM by transmitter levels and responses (Rioult-Pedotti et al. 2007; Ganguly and Poo 2013). Therefore, memory is stored as specific patterns of activity in neural networks (Citri and Malenka 2008). Short-term synaptic plasticity is believed to have a decisive function in filtering for efficient information processing. At the presynaptic level it is induced by the increased release of neurotransmitters and by protein modification. At the postsynaptic terminal basic mechanisms as desensitization and retrograde messengers might be involved. Long-term synaptic plasticity refers to the encoding of memories into neural circuits via enduring activation. One mechanism behind synaptic plasticity is the concept of long-term potentiation (LTP) and long-term depression (LTD) (Cooke 2006; Ganguly and Poo 2013). While short high- 
frequency presynaptic firing results in the induction of LTP, lower frequencies induce LTD (Feldman 2012; Andersen et al. 2017). A presumed fundamental mechanism for the creation of memories is the alteration of preexisting relations induced by LTP and LTD as well as the reconstitution and eradication of synaptic connections (Poo et al. 2016). Interestingly, synaptic strength also depends on the spike timing labelled as the concept of spike-timing dependent plasticity (STDP) (Park et al. 2017). Recent research revealed that there are obligatory time windows defining the maximum distance between pre- and postsynaptic spiking providing a very short period of $20 \mathrm{~ms}$ for LTP and an interval not longer than 100 ms for LTD (Poo et al. 2016). The order of spiking is causal as presynaptic before postsynaptic spikes induce LTP, while postsynaptic before presynaptic spikes lead to LTD (Feldman 2012; Andersen et al. 2017). Additionally, neuromodulators such as norepinephrine and dopamine can switch LTD signals into LTP (Andersen et al. 2017). For LTM encoding and learning, LTP and LTD are both utilized (Citri and Malenka 2008; Ganguly and Poo 2013). Following the multifactor plasticity rule, other factors such as firing rate and postsynaptic voltage are involved as well (Feldman 2012). All mechanisms lead to synaptic changes regarding existing synapses and result in new cell-cell connections (RioultPedotti et al. 2007; Bailey et al. 2015). Hence, changes in signaling induce the release of transcription factors and gene products leading to synaptic changes such as detected in the increase of the synaptic arbor in sensory neurons. Recent investigations suggested that the modulation in dendrites and the addition of spines might be based on the close localization of multiple mitochondrial ribonucleic acid (Poo et al. 2016; Bono et al. 2017). A major role plays the reorganization of the cortex after injury when plastic effects lead to the overtaking of specific functions by neighbored areas (Ganguly and Poo 2013; Nudo 2013). However, it can also lead to illness as in overexpression in case of focal dystonia, especially in musicians, and chronic pain (Pascual-Leone et al. 2005; Ganguly and Poo 2013). In general, many psychiatric diseases such as Alzheimer's disease, depression and Parkinson's disease are associated with irregular neuronal circuits (Cooke 2006; Citri and Malenka 2008; Brignani et al. 2013). Neuroplasticity after stroke induces changes not only in dendritic formation but also in axon sprouting (Ganguly and Poo 2013). An important issue rising with knowledge about human neuroplasticity is its opportunity for establishing new diagnostic and therapeutic tools. During the last decades non-invasive brain stimulation methods gained a particular importance in that context as it allows access to the investigation of underlying mechanisms and possible interventions (Ganguly and Poo 2013; Fertonani and Miniussi 2017). 


\subsection{Face perception}

Among all types of memory, facial memory is of particular importance due to its immense and unique significance for human communication and social behavior. Undoubtedly, the human face is elemental to interpersonal relationships and communication. Face perception serves as an obligatory requirement for the comprehension of emotions through face expression and is fundamentally important for identifying and distinguishing between people (Leopold and Rhodes 2010; Martinez 2017). Even new-born children are reacting differently to faces in contrast to other visual stimuli, which is highlighting the considerable weight of faces in the history of mankind (Little et al. 2011). Since long it is believed that processing faces relies on different, highly sophisticated mechanisms in contrast to object recognition, but revolutionary evidence came from a study using functional magnetic resonance imaging (fMRI) by Kanwisher and colleagues (Kanwisher and Yovel 2006). They could identify a cortical area that was activated in 12 of 15 subjects by looking at human faces in contrast to objects. The responding area named fusiform face area (FFA) was determined to be located in the fusiform gyrus (see Figure 5). The specificity of this area to be exclusively responding to faces was proved by also testing it against other parts of the human body as e.g. hands or by removing the hair to prove it as a unique processing area of face recognition rather than head recognition. Additionally, they could confirm lateralization by a greater activation of the right versus the left fusiform gyrus in most subjects. Nevertheless, a study was conducted with regard to the importance of the connectivity between both fusiform gyri in the left and right hemisphere for the consolidation of facial memory (Geiger et al. 2016). They found out that higher activity between the left and the right FFA after encoding leads to increased stability in memory. Moreover, there were detected connections to the amygdala, the hippocampus, and the inferior frontal gyrus, when higher connectivity was related to better memory performance. While the anterior segment of the hippocampus was mainly involved during encoding, the posterior segment had a causal role for the retrieval of faces. Accordingly, it was shown that interactive processes between the FFA and the posterior hippocampus occurring 15 minutes after the encoding of a facial stimuli led to higher performance in retrieval. 


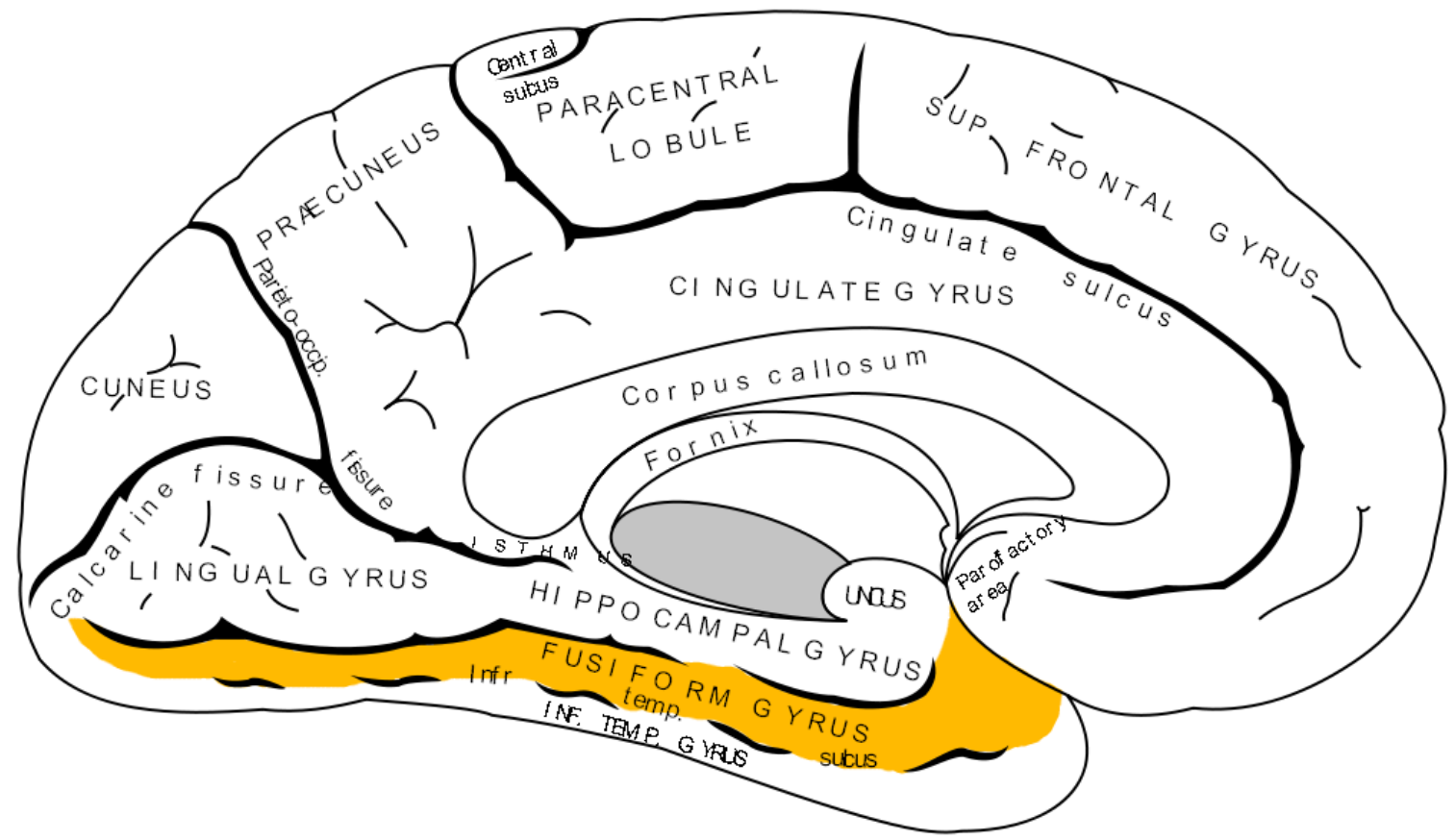

Figure 5: Schematic anatomical architecture of the brain (cross section). The yellow mark labels the fusiform gyrus that is related to face perception. The illustration is distributed under the CCO license. (https://commons.wikimedia.org/wiki/File:Gray727_fusiform_gyrus.png)

However, face perception is a highly complex process involving more than one cortical structure. When watching a face, the first specific area that the representation is attending is the occipital face area (OFA) in the lateral part of the occipital lobe (Pitcher et al. 2011; Li et al. 2016). This specialized area was found to be larger and more remarkable in the right hemisphere. Input into the OFA comes from early visual cortical areas, when complex face perceptions are holistically merged for the first time (Pitcher et al. 2011). In contrast to the FFA, that processes faces in a holistic way, the OFA is also causal for the perception of face parts (Arcurio et al. 2012). Initial evidence for the OFA as the first stage of face perception came from patients with an impaired right OFA but preserved right FFA, suffering limitations of recognizing faces, while object recognition and discrimination were still on a regular level (Pitcher et al. 2011). In addition, experimental application of transcranial magnetic stimulation (TMS) on the right OFA could thereupon induce an impairment of face discrimination in contrast to tasks with objects or other parts of the body. Facial representations are then believed to be passed to the FFA. After facial representations have undergone the OFA and the FFA they attend higher cortical areas such as the superior temporal sulcus (STS), principally its posterior part (Corrow et al. 2016; Kadipasaoglu et al. 2017). This region is related to various social perceptions, e.g. faces and voices. Here, faces are analyzed on a higher level also with regard to emotional expression and gaze (Leopold 
and Rhodes 2010). Additionally, human motion and understanding behavior and language are associated with processing in the STS, sometimes with coinciding areas (Deen et al. 2015). Altogether, OFA, FFA, and STS form the core regions being selective for facial stimuli, whereby the FFA shows the highest activation for faces (Tsao and Livingstone 2008). The fact that faces are processed in a holistic way with internal patterns for upright faces and not by analyzing face parts separately is also proved by the investigation of the Thatcher Effect (Adachi et al. 2009; Little et al. 2011). It shows the difficulty to notice changes in an inverted face while they would immediately be perceived in an upright one.

However, there is evidence that there are also other brain areas involved in the processing of faces such as the amygdala, the anterior temporal lobe, and the PFC (Elbich and Scherf 2017). Additionally, the hippocampus plays a causal role in the storage of faces as its left and right anterior segmentations are believed to be important for face processing and face discrimination (Robinson et al. 2015). After processing, facial memory is stored in the episodic memory. The patient H.M. (see Chapter 1.1.3) was not able to complete tests dealing with facial identification and recognition revealing the importance of the MTL for the storage of episodic memory involving faces (Eichenbaum 2017). Face perception comprises the combination of two opposing processes (Tsao and Livingstone 2008). On the one hand, it requires the detection of a face in contrast to objects and other parts of the body by using a universal pattern for recognizing the typical T-shaped form of a face consisting of the eyes, mouth, and nose. On the other hand, there is a demand for classification and identification by observing differences between faces and comparing the presented face to internal templates to allocate the face to a character.

As most studies are conducted on verbal memory, even though every-day episodic memory is much more concerned with visual stimuli, there is a need for further research on memory about it with special respect to faces as a basic tool for interpersonal skills and social communication (Rugo et al. 2017).

\subsection{Temporo-parieto-occipital area}

In the study of anatomical correlates of face processing the cortical structure of the temporoparieto-occipital (TPO) area has received considerable critical attention. It involves components of three brain lobes and serves as a highly connected integration area (De Benedictis et al. 2014). It describes the area at the occipital end of the lateral sulcus that incorporates a part of the parietal, occipital, and temporal lobe. Characteristic of the parietal lobe is the processing of sensory information, while the occipital lobe is related to vision. 
The temporal lobe incorporates different structures and adds meanings to auditory and visual input. The TPO area involves the region where the three lobes meet and serves as an association area combining functions and signals for higher cognitive purposes. While in the past the TPO area was mainly related to language perception, nowadays it was proved to be an important element in many higher processing operations such as coordination of cognitive functions (Wang et al. 2016). In fact, this area is important for the processing of auditory, visual, and somatosensory stimuli. Important linguistic abilities are related to the TPO area such as language, writing and reading (De Benedictis et al. 2014). Besides musical memory and calculative skills it is also involved in face and object recognition. There was found lateralization for each hemisphere indicating that the left TPO area is mainly involved in language, while the right TPO area shows higher activity for face processing.

\subsection{Brain oscillations}

Coming from the anatomy to the physiology of the human memory, brain function does not simply rely on the activity of individual cells but is dependent on connected cell assemblies with efficient communication mechanisms. A fundamental mechanism for communication are brain oscillations that are occurring ubiquitously in the brain (Buzsáki et al. 2013). They are an elementary means for enabling neurons and neuronal networks to communicate and to transfer and integrate information (Lin and Scott 2012; Buszáki and Schomburg 2015). On the one hand, oscillations can encode information and induce plasticity and memory formation (Lopes da Silva 2013; Pevzner et al. 2016). On the other hand, they are also able to manipulate brain states or to reinforce an already existing state. Oscillations are formed as a reoccurring periodic signal with various frequencies (Fell and Axmacher 2011). They are characterized by their phase, amplitude, and frequency. The spectrum ranges from lower frequencies as delta $(<4 \mathrm{~Hz})$ and theta $(4-8 \mathrm{~Hz})$ to higher frequencies such as alpha $(8-13 \mathrm{~Hz})$, beta $(13-30 \mathrm{~Hz})$, and gamma that can be separated into low gamma (30 $70 \mathrm{~Hz}$ ) and high gamma $(70-140 \mathrm{~Hz}$ ) (Moran and Hong 2011). Frequencies above $140 \mathrm{~Hz}$ are summarized as the "ripple range" (Korotkova et al. 2018). With regard to the origin of neural oscillations, there is still little comprehension about the underlying mechanisms or potential pacemakers. There is evidence that non-pyramidal cells, more precisely inhibitory interneurons, play a major role in the formation of oscillations (Moran and Hong 2011; Lin and Scott 2012; Buzsáki et al. 2013; Cohen 2017). Inhibitory interneurons using gamma aminobutyric acid (GABA) as a transmitter are connecting many excitatory pyramidal cells in order to influence their activity. Oscillatory synchrony is assumed to be based on their 
influence on the membrane potentials of pyramidal cells. Accordingly, the length of inhibitory post-synaptic potentials slows down the frequency of the corresponding oscillation.

For the communication of different brain regions phase synchronization of their oscillations is crucial (Fell and Axmacher 2011; Bonnefond et al. 2017). This phenomenon is referred to as "communication-through-coherence", as it facilitates interaction by creating time windows when sensitivity for informational input is maximal (Fries 2005; Bonnefond et al. 2017). There is evidence that precision of phase synchronization increases the rate of later remembered information during encoding. A possible mechanism behind this investigation is that synchronization ensures that neuronal activity in one region leads to the adaption of activity in another area that is coherent in oscillatory activity. Moreover, the induced precision then leads to inputs occurring at the excitatory phases of the oscillations where it is most effective (Lisman and Jensen 2013). Additionally, it provides the possibility to exempt neural signals from noise that is transferred asynchronously (Nyhus and Curran 2010). This efficiency in energy is of fundamental importance as 50 percent of the brain's power is spent on information transmission (Schnitzler and Gross 2005). Important conditions for successful coherence are correlating frequencies and phases as well as an appropriate lag in transmission (Fries 2005). In contrast, the gating-by-inhibition hypothesis states that the blocking of regions which are not necessary for the performance of the task allows optimal transfer of information (Jensen and Mazaheri 2010; Bonnefond et al. 2017). Apart from their causal role for neural communication brain oscillations also support neural plasticity (Fell and Axmacher 2011). STDP is increased when phase synchronization is established due to the fact that action potentials induced by excitatory cells emerge more likely during the depolarization (Nyhus and Curran 2010; Fell and Axmacher 2011).

Different frequencies were investigated to have different functions in neural communication. The shorter the distances between neurons, the faster occurs oscillatory activity, while more distant cell assemblies communicate via slower frequencies (Nyhus and Curran 2010). Distinct brain functions and brain regions use different oscillatory frequencies, nevertheless, one frequency is not unique to one area or cognitive function. A frequency widely investigated in cognitive functions is the theta frequency, in rats defined in a range from 6 to $10 \mathrm{~Hz}$, while in humans they comprise a slower range down to $4 \mathrm{~Hz}$ (Buzsáki et al. 2013; Hanslmayr et al. 2016). This phenomenon is most likely based on the divergent brain size suggesting that an increase in size is related to slower frequencies due to further distances that need to be bridged. 
Theta oscillations are occurring in various brain regions and the underlying pacemaker rhythms are still not fully elucidated. Hippocampal theta rhythm is most likely originated in various brain structures, e.g. in the prefrontal and entorhinal cortex (Düzel et al. 2010). Additionally, the medial septal nucleus exhibits theta rhythm and is believed to be an important theta generator of the hippocampus, while the nucleus basalis is responsible for the synchronization of theta oscillations in cortical regions (Lisman and Jensen 2013). Lesion studies confirm the correlation of the medial septum and fornix with theta origin leading to significant impairments in memory tasks testing spatial memory (Korotkova et al. 2018). There are also other subcortical nuclei being connected to control mechanisms of theta rhythm such as the supramammillary nucleus or the nucleus incertus. These structures are counterparts to the median raphe nucleus that is inducing hippocampal non-theta states.

Theta oscillations are assigned to various cognitive abilities such as attention, learning, memory, synaptic plasticity, arousal, and anxiety behavior (Brignani et al. 2013; Kleinert et al. 2017; Korotkova et al. 2018). An important finding was the discovery of place cells in the rodent's hippocampus that are associated with spatial navigation and gained particular attention (Dragoi and Buzsáki 2006). When the rat moves through a place field, theta oscillations constantly occur in the hippocampus and increase in frequency and amplitude with ascending speed and age (Düzel et al. 2010; Korotkova et al. 2018). With starting to pass into a defined part of the location, place cells start to fire at gamma frequencies occurring at the peak of each theta cycle (Dragoi and Buzsáki 2006). By moving further gamma oscillations shift phases. This phenomenon is described as "theta phase precession". Matching investigations about theta rhythms were also made in the field of human spatial orientation (Düzel et al. 2010). Interestingly, theta reflects the familiarity of the environment. In fact, foreign locations induce lower theta frequencies in rats in comparison to familiar environments.

Results from earlier studies also demonstrate a strong and consistent association especially between oscillations in the theta and gamma range and memory function (Buzsáki and Silva 2012; Brignani et al. 2013; Hanslmayr et al. 2016; Albouy et al. 2017). They are assumed to support communication between brain regions and seem to be determinant for the encoding of spatial impressions and objects (Düzel et al. 2010). For instance, brain activity during spatial memory tasks showed theta coherence between the hippocampus and other brain areas such as the PFC. The oscillatory synchrony between the PFC and the hippocampus varies depending on the context. During memory retrieval oscillations in the PFC go ahead of oscillations in the hippocampus while this is reversed during a memory delay indicating the direction of information transfer (Eichenbaum 2017). Moreover, there is evidence that 
theta phase synchronization between the frontal and temporal cortex as a sign for neural communication is important for the formation of long-term memories (Fell and Axmacher 2011). This is based on the organization of simultaneous synaptic input that leads to the facilitation of depolarization and consequently to higher efficiency in inducing action potentials and exciting postsynaptic neurons. Besides phase coherence, oscillatory activity can provoke the firing of neurons as subthreshold activity can be added by synchronized organization to gain suprathreshold activity (Pevzner et al. 2016). Furthermore, oscillatory activity in the theta range is not only important for long-range brain communication, but it is also associated with the induction of synaptic plasticity (Fell and Axmacher 2011; Berens and Horner 2017). Excitatory inputs during the peak of theta oscillations then induce LTP, while when occurring at the trough they induce LTD. During synchronized excitatory phases LTP enables the formation of LTM.

Recent work has shown that theta-gamma phase-amplitude coupling is crucial for working memory (Lisman und Jensen 2013; Alekseichuk et al. 2016). The concept describes the occurrence of theta and gamma frequencies operating in interaction and assumes that memory items are encoded in gamma cycles, which are then organized in theta phases. This mechanism secures the maintenance of several objects and their correct temporal order (Düzel et al. 2010). The concept of theta-gamma coupling was strengthened by a study where they used stimulation with transcranial alternating current to slow down the theta rhythm in order to increase the number of gamma cycles fitting into it and expand working memory load (Vosskuhl et al. 2015). Accordingly, this mechanism plays a major role for the information transfer between neocortex and hippocampus and therefore for consolidating STM into LTM (Guderian et al. 2009; Lisman and Jensen 2013). Theta-gamma coupling has been discovered in several brain regions revealing it as a fundamental mechanism for memory encoding (Lisman 2010; Belluscio et al. 2012). There is also evidence for a positive correlation between theta amplitude and the subsequent memory effect (defined as successful memory encoding based on a particular neural activity pattern), as higher amplitudes shortly before the presentation of the stimulus led to a higher amount of later correctly recalled words and therefore must be connected to encoding mechanisms (Guderian et al. 2009). A similar correlation was shown for theta synchronization between the hippocampus and rhinal cortices (Düzel et al. 2010). Various studies revealed that increased theta power in the MTL provokes enhanced learning achievements, e.g. improved episodic memory encoding (Düzel et al. 2010; Berens and Horner 2017). Accordingly, theta seems to operate as a coordinator for transferring and integrating information from distinct brain regions and for securing the exact timing of all inputs to induce LTP (Buszáki and Schomburg 2015; Berens and Horner 
2017; Clouter et al. 2017). In fact, oscillatory activity is crucial for episodic memory (Hanslmayr et al. 2016). As aforementioned, theta-gamma coupling is responsible for the binding of stimuli into internal representations and this also applies for the binding of episodes in the MTL. Moreover, oscillatory activity in the theta range was proven to be crucial for two memory processes. On the one hand, it is highly correlated with successful memory encoding, e.g. by the temporal organization of activity during LTM formation (Antonenko et al. 2016; Berens and Horner 2017). On the other hand, theta is also highly important for later correctly retrieved information as slow theta predicts successful recall in contrast to fast theta that was recorded in relation to processes of interference (Lisman and Jensen 2013; Pastötter and Bäuml 2014). It was shown that stimulation in one phase can induce encoding while in another it provokes retrieval supporting the fact that these processes depend on different theta phases (Korotkova et al. 2018). There is given evidence from a study by Clouter et al. that episodic memory inputs synchronized in the theta-band are better memorized in contrast to asynchronously presented inputs (Clouter et al. 2017). Additionally, they tested theta synchronization in contrast to other frequencies and found this effect as unique for theta compared to delta- or alpha-modulated stimuli.

The importance of functioning synchronization in the human brain becomes obvious with the pathological mechanisms behind different neuropsychological diseases. For example Alzheimer has been shown to correlate with the functional interruption of brain networks and reduced synchronization within various frequency bands (Schnitzler and Gross 2005). Moreover, investigation of underlying mechanisms behind schizophrenia reveals failure in gamma synchronization followed by impaired connectivity between brain areas (Schnitzler and Gross 2005; Lopes da Silva 2013). At last, there are several motor disorders such as Parkinson's disease, which reveal a failure of long-range synchronization (Schnitzler and Gross 2005).

\subsection{Neuroimaging - electroencephalography (EEG)}

The electroencephalography (EEG) is a non-invasive electrophysiological technique first established by Hans Berger in the 1920s for the recording of neural activity (Herrmann et al. 2016a). For the measurement small electrodes are attached to the scalp to record brain oscillations reflecting synchronized and reoccurring activity of neuronal assemblies (Moran and Hong 2011; Jackson and Bolger 2014). This method cannot measure single neuronal activity but only the summation of extracellular currents of large populations of pyramidal cells (Fell and Axmacher 2011; Cohen 2017). Accordingly, the summation of postsynaptic 
potentials of many pyramidal cells forms local field potentials (LFP) that are detected by EEG. Moreover, brain activity can only be recorded when an electrode is not equidistant but closer to one pole making a dipole measurable (Jackson and Bolger 2014). Within the brain, the neural signal is transferred via volume conduction and between different volumes it is transmitted through the mechanisms of capacitance. Therefore, the finally recorded signal highly underlies the physical composition and the electrical conductivity of the different brain tissues (Lopes da Silva 2013; Jackson and Bolger 2014). EEG data provides information about brain oscillations that are a correlate for different neuronal processes with a scope ranging from motor to cognitive processes (Cohen 2017). Regional frequency-specific modifications in power in reaction to a stimulus are often reported as event-related synchronization (ERS) indicating an increase in oscillatory power and event-related desynchronization (ERD) referring to the opposite (Fell and Axmacher 2011). An outstanding advantage of EEG is its high temporal resolution. Furthermore, its noninvasiveness provides the possibility to get insights into the functioning of human brains not only in diseased but also in healthy people expanding the substrate for investigation in neurophysiology. Consequently, EEG is a highly appropriate method to elucidate dynamic cognitive processes connected to the encoding of facial stimuli.

\subsection{Neuromodulation techniques}

\subsubsection{History of the application of electrical current on the human brain}

The idea of using electric current in order to influence the human brain is not new as far as the origins of applying electricity on humans date back to the antiquity. Long ago in $46 \mathrm{AD}$ under the Roman Emperor Claudius, first discoveries of electric fish like electric eel and crampfish were made by Scribonius Largus in conjunction with pain (Priori 2003; Brunoni et al. 2012). He found out that a torpedo fish placed on the scalp relieved the pain of headache and thereby established a new treatment for pain during the ancient world. Other researchers coming to the same solutions were Pliny the Elder and Galen of Pergamum. The latter constituted the effect on the fish's torpor, numbness, and narcotic effect. Ibn-Sidah, living in $11^{\text {th }}$ century, also used a catfish for treating epilepsy. Around 1780, Luigi Galvani made a historical discovery: he found out, that the stimulation of a frog's leg by electric current caused muscle contraction, which was followed by other experiments using galvanic current on animal electricity (Piccolino 1998). His nephew Aldini expanded the scope of application by stimulating patients who suffered from melancholia (Zaghi et al. 2010). Applying the current on his own head at first, he reported insomnia as a consequence next 
to an uncomfortable feeling (Priori 2003). Another milestone in the history of brain stimulation was the investigation of the first battery by Volta around 1800, which cleared the way for an increasing research with focus on the impact of electric current on the brain (Paulus 2011). Until the middle of the $20^{\text {th }}$ century the more qualitative experiments and the missing protocols and measuring methods complicated the conditions for progressing investigation of brain stimulation because of inconsistent results that were neither reliable nor reproducible. While the investigation of high-intensity transcranial stimulation by Bini and Cerletti brought up a new treatment for depression of mental disorders that was called electroconvulsive therapy and is still used today, experiments with low-intensity stimulation became popular not until the 1960s again (Priori 2003). In 1963, Limoge made the causal discovery of the impact of stimulation in anesthesia using low-intensity alternating transcutaneus cranial electrical current (Limoge et al. 1999). The so-called "Limoge's current" seemed to be a method for anesthesia being gentler due to its minor demand for narcoleptics. During the second half of the $20^{\text {th }}$ century there were many experiments with various main focuses making use of transcranial current (Priori 2003). One year later, Bindman et al. applied direct current to the scalp of rats and detected enduring effects beyond the duration of stimulation (Bindman et al. 1964). This was followed by further studies investigating the effect of direct current on patients with psychological diseases (Lefaucheur et al. 2017). The important reversal point was reached with the development of TMS when weak magnetic fields were discovered to have an impact on cortical excitability by Barker in 1985. It is a non-invasive method working with magnetic pulses applied on the scalp and resulting in an excitation of cortical neurons changing cortical plasticity (Lage et al. 2016). It was the first alternative to other stimulation methods at that time since it was painless and did not require surgery (Ziemann 2017). This induced a renewed interest in the investigation of the impact of electric current on the brain also due to improved protocols and increased reproducibility of experiments (Zaghi et al. 2010). The last decades then led to the development of noninvasive brain stimulation (NIBS) and the application of current on the human scalp in order to manipulate cortical excitability and neuroplasticity, not only to investigate brain functions but also to find new therapeutic strategies for neurological diseases (Zaghi et al. 2010; Santarnecchi et al. 2015).

Nowadays there are different forms of manipulating the brain by using different techniques collectively referred to as 'neuromodulation'. Methods can be divided into invasive and noninvasive methods. Invasive methods include particularly deep brain stimulation (DBS), which describes a neuro-surgical method where a stimulation electrode, which is connected to a stimulator that is implanted as well, is placed at a specific part in the brain - typically at the 
basal ganglia nuclei - and delivers pulses of direct current to the targeted area (Rossi et al. 2016). This technique was already brought up in the 1980s and the scope of application is still growing (Gildenberg 2005; Vedam-Mai et al. 2014). The intervention is reversible and intends to influence a neural target that is causal for the disorder's symptoms that the patient is suffering from in order to improve them. Even if there is not much known about the theoretical background of the mechanisms, DBS is commonly used in various different diseases such as tremor or chronic pain (Gildenberg 2005; Rossi et al. 2016). Non-invasive methods are techniques based on the application of low-intensity transcranial electric stimulation (tES) on the scalp and mainly refer to three methods: At first, transcranial direct current stimulation (tDCS) delivers constant direct current. Secondly, transcranial random noise stimulation (tRNS) transmits randomly distributed currents with a frequency spectrum between 0.1 and $600 \mathrm{~Hz}$. At last, transcranial alternating current stimulation (tACS) is a method where a sinusoidally oscillating current is entraining brain oscillations. Accordingly, small electrodes are applied to the scalp in order to deliver different forms of current with respect to the method on the brain, particularly on the underlying area as the region of interest. In contrast to the effects occurring during the stimulation summarized as online effects, there can also arise after-effects from minutes to even hours beyond the application (Lefaucheur et al. 2017). While online effects might be caused by direct influence of the stimulation on membrane potentials, after-effects could be a consequence of brain plasticity and altered synaptic connections with long-lasting changes of neuronal excitability (Antal and Herrmann 2016; Yavari et al. 2017).

Today, non-invasive brain stimulation techniques (NIBS) are used for different interests. On the one hand, they are applied in order to investigate the physiology of the human brain and the stimulation's impact on the neuronal activity and plasticity (Yavari et al. 2018). On the other hand, tES is regarded as a promising means to treat neurological and psychiatric disorders (Abd Hamid et al. 2015; Fertonani und Miniussi 2017; Lefebvre and Liew 2017; Wortman-Jutt and Edwards 2017). Since for our experiments tACS was the method of choice as meeting our requirements most, the next section will provide detailed information and enhanced insights into scientific findings and recent studies on the field.

\subsubsection{Transcranial alternating current stimulation (tACS)}

In the last decade, the use of transcranial alternating current stimulation (tACS) as a noninvasive stimulation method gained growing interest. Already in 2006, Marshall et al. combined tDCS with low-frequency tACS $(0.75 \mathrm{~Hz})$ to induce a boost in memory during non-rapid-eye-movement sleep (Paulus 2011; Antal and Paulus 2013). Unfortunately, the 
study did not include necessary control experiments for verifying the mechanism of memory improvement. In a pioneer study in 2008, Antal et al. applied tACS reclusively with several frequencies between 1 and $45 \mathrm{~Hz}$ on the motor cortex with an intensity of $0.4 \mathrm{~mA}$, that resulted in an improved implicit motor learning effect for the frequency of $10 \mathrm{~Hz}$ (Antal et al. 2008; Lefaucheur et al. 2017). Other studies followed with focus on diverse abilities and also diseases (Abd Hamid et al. 2015). With tACS, a weak biphasic and sinusoidal electric current is externally applied by electrodes on the scalp in a biphasic and sine-wave form intending to reach the underlying brain area and entrain brain oscillations with adapting the internal rhythm to the externally applied frequency (see Figure 6) (Paulus 2011; Fröhlich et al. 2015). The adapting internal oscillations lead to endogenous synchronization not only between neurons being located in the targeted area but also between distant brain areas (Schutter 2014).

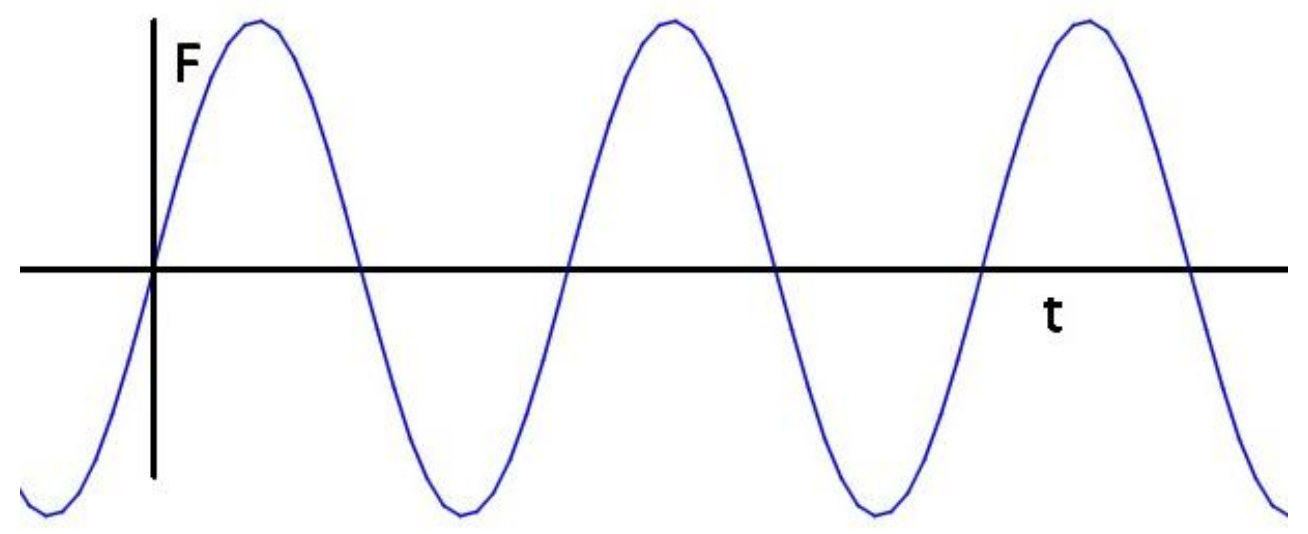

Figure 6: Transcranial alternating current oscillates with a sinusoidal wave form. The y-axis depicts the amplitude $(\mathrm{F})$, the $\mathrm{x}$-axis displays the time (t). (Own illustration.)

There are several determinant parameters for the application of tACS. Next to the phase (the temporal correspondence between the phase of the endogenous rhythm and the external alternating stimulation), the frequency and intensity are crucial for the induction of oscillatory effects (Antal and Paulus 2013; Antal and Herrmann 2016). While a frequency lower than the endogenous rhythm is hypothesized to slow down internal brain oscillations, a higher frequency might speed them up (Antal and Herrmann 2016). Different frequencies were investigated to have diverse effects on human behavior. Regarding motor performance, stimulation in the alpha range was found out to improve it; in contrast beta stimulation worsened the outcome (Antal and Paulus 2013). While in the beginning tACS studies mainly focused on motor functions, recent studies expanded the scope of application to cognitive 
processes and memory function. Especially theta and gamma frequencies were identified to have a highly relevant effect on attention and memory (Antal and Paulus 2013; Schutter 2014). As an example, stimulation of the frontal cortex in the gamma range enlarges the cognitive load and relieves the access to it (Schutter 2014). In contrast to that are frequencies exceeding the frequency spectrum of EEG. The so called "ripple range" involving high frequencies from 80 to $250 \mathrm{~Hz}$ is not only supposed to affect the motor cortex (M1) in an intensity-dependent manner, these frequencies are also considered to be associated with memory encoding (Antal and Paulus 2013; Lefaucheur et al. 2016). Finally, stimulation in the high $\mathrm{kHz}$ dimension of about $200 \mathrm{kHz}$ is assumed to have an effect in cancerous diseases (Antal and Paulus 2013). Interestingly, the application of tACS does not only entrain internal brain rhythms in the applied frequency range but also in frequencies that are subharmonics to the externally applied and closer to the internal frequency of the targeted area (Herrmann et al. 2016b)

Another fundamental parameter for the application of tACS is the phase of stimulation (Antal and Paulus 2013). For instance, a phase-specific effect was detected during the stimulation of left frontal and parietal targets with a frequency of $6 \mathrm{~Hz}$. An in-phase stimulation of the mentioned brain areas led to an enhanced performance of working memory, whereas the application of anti-phase stimulation deteriorated the outcome (Polanía et al. 2012). Another study elucidated that the phase and the optimal listening behavior are coupled indicated by decreased response times occurring with the individual optimal delta phase (Henry and Obleser 2012).

In fact, also the intensity plays an important role for the induced after-effects of tACS (Antal and Paulus 2013; Antal and Herrmann 2016). While an intensity of $0.2 \mathrm{~mA}$ has no measurable effect, $0.4 \mathrm{~mA}$ appears to be inhibitory leading to the conclusion that inhibition might be related to lower intensities. Besides, there are other relevant influential aspects, for example electrode size and shape as well as the montage of the device. More factors such as duration still need to be investigated with regard on their importance and impact on possible aftereffects (Antal and Paulus 2013).

Even after one decade of investigation the mechanisms behind tACS are still debated. Indeed, there are some suggestions about the modification of biochemical equilibriums, which might be causal for the induction of after-effects by provoked synaptic plasticity, possibly due to calcium accumulation in the presynapsis (Antal and Paulus 2013). However, the dominant theory of tACS suggests an entrainment of brain oscillations to the externally applied oscillating current as the mechanism of action. The entrainment of brain oscillations 
is determined by the electric polarization of neurons, which is permanently changing its polarity due to the alternating current and might lead to the periodical adaptation of transmembrane potentials (Fröhlich et al. 2015). This also serves as an explanation for the effect of tACS in neurological diseases that are often connected with faulted and desynchronized internal brain oscillations and might be resynchronized by externally applied alternating current (Abd Hamid et al. 2015; Fröhlich et al. 2015; Antal and Herrmann 2016). In contrast to TMS, where short high-intensity impulses influence the underlying cortex in a suprathreshold manner, tACS does not induce action potentials but has subthreshold effects on neuronal resting membrane potentials with impact on firing rates due to the application of low intensities (Yavari et al. 2018). The alternating current entrains internal brain oscillations and leads to synchronization within and between brain networks. While altered membrane potentials are a plausible explanation for online effects, they do not fully explain after-effects. Consequently, it is suggested, that tACS also induces morphological adaptation processes by interfering with cellular mechanisms on a genetic level such as the regulation of genes and the induction of protein synthesis (Cirillo et al. 2017).

\subsubsection{Current state of the art}

In the last years, tACS has attracted increasing attention after some studies revealed promising results (Antonenko et al. 2016). Until today, there has been published various literature investigating the effect of tACS on attention, perception, motor function as well as on higher cognitive functions, memory, and learning (Tavakoli and Yun 2017). After the discovery of the impact of tACS on implicit motor learning by Antal et al. in 2008, many other studies followed concerning stimulation effects in motor functions. For instance, Schutter and Hortensius applied delta, theta, alpha, and beta frequencies on the left and right motor cortex with an intensity of $1 \mathrm{~mA}$ for 10 minutes (Schutter and Hortensius 2011). The results revealed that both theta and beta frequencies increased the cortical excitability of the primary motor cortex. Due to the promising findings, investigators gradually expanded the scope from motor cortex to other brain areas and related functions. Consequently, there were also conducted many studies with regard to cognitive skills (Abd Hamid et al. 2015; Antonenko et al. 2016). As theta oscillations are highly related with memory formation and plasticity effects, several studies investigated the impact of theta-tACS on memory processes. To test the impact of tACS on the fluid intelligence and executive control Pahor and Jaušovec conducted a study with a problem-solving and visual-spatial reasoning task (Pahor and Jaušovec 2014). They applied theta-tACS with a frequency of about $5 \mathrm{~Hz}$ at the left frontal and parietal cortex with an intensity from 1 to $2.2 \mathrm{~mA}$ for 15 minutes. Right after, all subjects 
had to perform the tasks. The recorded EEG revealed an increase in frontal theta power and a decrease in posterior alpha power. The parietal stimulation group showed improved performance in comparison to the frontal and the sham stimulation group. Pahor and Jaušovec interpreted the results as an indicator for the increase in working memory capacity and as an after-effect of theta-stimulation. Furthermore, it was derived a connection between frontal theta-tACS and attentional control. In 2015, Vosskuhl et al. also investigated the impact of tACS on the capacity of working memory (Vosskuhl et al. 2015). Referring to the assumption that the memory capacity is based on the theta-gamma coding theory, the stimulation frequency was always chosen below the individual theta frequency that was detected by an EEG measurement before the application of stimulation and never exceeded $5 \mathrm{~Hz}$ (Lisman 2010; Vosskuhl et al. 2015). This was intended to downregulate every subject's theta frequency and thereby make more gamma cycles fit into one theta cycle in order to extend memory capacity. In comparison with sham an alternation of performance under stimulation was seen in the forward digit span indicating an increase in STM capacity as an online effect. A recorded after-effect was the increase of the theta-amplitude that stayed elevated even beyond the duration of stimulation. Another study focusing on the influence of tACS on cognitive functions was carried out by Santarnecchi et al. in 2016. With an abstract-reasoning test, a delayed-match-to-sample test, and a control task the response time was measured while the PFC was stimulated with gamma-tACS with a frequency of $40 \mathrm{~Hz}$ and an intensity of $0.75 \mathrm{~mA}$ for 30 minutes (Santarnecchi et al. 2016). For control, it was also applied a theta-tACS of $5 \mathrm{~Hz}$, tRNS, and sham. As a result, the application of gamma-tACS unveiled shorter responding times for this specific frequency. The importance of theta oscillations for memory processes and its causal role in phase synchronization between frontal and parietal cortices for memory processes was investigated by Polanía et al., who applied synchronized theta-tACS to both brain regions and recorded significantly improved reaction times in a delayed letter recognition task (Polanía et al. 2012). A recent study by Javadi et al. focused on the encoding into and retrieval from LTM (Javadi et al. 2017). Here, tACS was applied in the gamma frequency spectrum during encoding and retrieval to entrain internal brain rhythms. For the application of the same frequency it was found a significant effect in memory improvement in contrast to the utilization of different gamma-frequencies in encoding and retrieval processes. Altogether, these studies provide an insight into former literature proving the efficacy of tACS as a powerful tool not only for the investigation of causal relationships between brain oscillations and behavior. It is also a highly adequate technique to interfere with cortical activity and manipulate brain functions to affect memory capacity, performance, and reaction time. 


\subsubsection{Clinical applications}

Besides its promising potential to investigate the connection between specific brain rhythms and their associated brain functions, tACS also offers the possibility of application for therapeutic purpose (Vosskuhl et al. 2015). Oscillatory desynchronization as a potential cause for neurological dysfunction has been observed in several brain disorders such as Alzheimer's disease and Parkinson's disease. Consequently, tACS might have the potential as a new tool concerning the differential diagnosis of neuropsychiatric maladies and improve prevention as much as early detection before the onset of clinical signs and thereby an early intervention (Brignani et al. 2013; Naro et al. 2016). Furthermore, it offers the opportunity to resynchronize brain activity and relieve symptoms. There have been several studies testing the impact of tACS on different neurological and psychiatric disorders, for example on tinnitus, glioblastoma, or on schizophrenia (Lefaucheur et al. 2017; Sreeraj et al. 2017). In the main focus for the clinical application of tACS are various disorders that are connected to abnormal oscillation patterns such as Parkinson's disease or schizophrenia (Antal and Paulus 2013). By applying external current desynchronized oscillations shall be readjusted to their physiological rhythm (Fröhlich et al. 2015). In several studies it was also found evidence for the enhancement of visual damage by the application of alpha-tACS (Abd Hamid et al. 2015). While for patients suffering from visual restraints a reduced oscillatory power was measured, tACS could increase the alpha oscillations and lead to improved visual fields and better functioning in optic nerve neuropathy. These promising results reinforce the demand for further research on tACS not only for investigation approaches but also for the clinical application in the future.

\subsubsection{Safety aspects of tES}

In fact, there are many studies giving evidence for the safety of the application of transcranial electric current on the human scalp if the safety requirements are fulfilled (Abd Hamid et al. 2015; Fertonani et al. 2015; Santarnecchi et al. 2015; Bikson et al. 2016). All reported adverse effects (AE) in literature were on a mild sensational level. Only recently, Antal et al. published an extensive synoptic paper about the safety, the legal framework and application recommendations of tES (Antal et al. 2017). In a comprehensive review they evaluated AEs occurring simultaneously or subsequently to the stimulation in more than 18000 sessions since 2000. There were not reported any serious AEs in none of the sessions with regard to standard protocols applying current with an intensity below $4 \mathrm{~mA}$ and a duration shorter than 60 minutes. As mild side effects there were documented local skin sensations. Coupled with the induction of vasodilatation through the application of weak electrical current on the 
skin there may occur different skin irritations such as pinching, itching or tingling, potentially in combination with redness (Fertonani et al. 2015; Jamil et al. 2017; Reinhart et al. 2017). Furthermore, there have been described mild sensations underneath the electrode, albeit they are mentioned rarely for tACS and tRNS due to the continually changing current in contrast to tDCS where a constant current is applied (Schutter 2014; Fertonani et al. 2015). Actually, for having the same perception the intensity of tRNS must be three times higher than for tDCS, also indicating that a higher intensity aggravates the sensations as well as larger electrodes reinforces them (Fertonani et al. 2015). Conversely, there could not be observed an influence on sensations by other parameters such as the montage of the device on the scalp, timing or density of the current. Moreover, literature revealed other mild side effects such as weak headache, fatigue, and slight nausea (Schutter 2014; Abd Hamid et al. 2015; Antal et al. 2017). Also associated with tES is the occurrence of phosphenes during the application (Abd Hamid et al. 2015). Cutaneous as well as phosphene perception were shown to be dependent on the stimulation frequency with a peak in the beta and gamma frequency spectrum (Turi et al. 2013). Some studies included a montage of electrodes with local proximity to the mouth, tongue or trigeminal nerve, whereby a low percentage told about a metallic taste (Schutter 2014; Fertonani et al. 2015; Reinhart et al. 2017). Most of these sensations are only reported during and shortly after the stimulation, whether one week after no subject had perceptions related to the application of current anymore (Abd Hamid et al. 2015). In contrary, for tES there were not determined any AEs regarding cognitive or perceptual capabilities (Antal et al. 2017). Particularly with regard to tACS, Antal et al. confirmed the method as safe due to the absence of pathological, structural or functional changes in recordings of neither EEG and magnetic resonance imaging nor in enzymatic levels (Antal et al. 2017). Finally, they propose a checklist and a screening questionnaire prior to tES sessions for enhanced precaution and a standardized survey of possible AEs right after to maximize comparability between studies. Altogether and with regard to existing data, tACS is proven a safe technique with only mild side effects.

\subsubsection{Advantages and disadvantages of tES}

Transcranial electric current stimulation seems to be a rising method in research questions as much as in daily clinical practice. With regard to tACS, it appears to be a promising technique for the investigation of the relation between specific neural oscillations and the associated brain functions as well as the link between brain function and behavior not only in healthy but also in diseased people (Fertonani et al. 2015). In contrast to other techniques for measuring brain activity, tES is also able to not only prove correlation between specific 
oscillations and a related cortical function or behavior, but it is also suitable to create causality by interfering with oscillations and thereby changing a variable to observe altered cognitive functions and behavioral outcome (Tavakoli and Yun 2017). Compared to other stimulation methods tES enables to reach the target region without any detours via sensory cortices as in case of visual flickering (Brignani et al. 2013). A major benefit of tACS is its frequencyspecific entrainment of large neuronal networks at a nearly appreciable level (Tavakoli and Yun 2017). Additionally, it was shown to have a higher effect for entraining neuronal populations in contrast to other tES techniques such as tDCS. Concerning the application in patients, it also provides many benefits for clinical treatment as it is non-invasive and therefore makes a surgery obsolete (Abd Hamid et al. 2015). Additionally, the burden for patients during the treatment can be reduced as tES does not cause severe adverse effects and the device does not produce disturbing sounds (Antal and Paulus 2013; Schutter 2014). Patients who suffer from disorders where pharmacological options are missing or do not come into question due to drug intolerance, interactions with other medicaments or pregnancy might profit from this alternative (Brunoni et al. 2012). Moreover, it might not only have an effect in exclusive application but also in combination with other therapeutic treatments in order to enhance the curative effect. Devices for the application of weak current are smaller, low-priced and manageable, which also opens doors for the home-use of tES (Lefaucheur et al. 2017). On the one hand, patients with chronic disorders can exploit that because the probable need for regular sessions can be complied more easily. On the other hand, it is also affordable for poor countries where money for medical care and equipment is missing (Brunoni et al. 2012). However, the feasibility and the facilitated handling at home also contain risks due to improper use of non-professionals (Brignani et al. 2013; Lefaucheur et al. 2017). Additionally, the simple access and affordability also raise the question, whether healthy people might use it to enhance their cognition and leads to the ethical discussion of neuroenhancement (Santarnecchi et al. 2015). This is also of particular importance in relation with the still not fully elucidated mechanisms behind tES and nonlinearity and to a certain extent unpredictability of experimental results (Fertonani and Miniussi 2017). This enhances the demand for further studies to gain deeper knowledge about underlying mechanisms and improve application parameters.

\subsection{Aim of the study}

The topic of memory has gained growing interest in the last years. While working memory, attention, and cognitive control were target in various studies, surprisingly, effects of tACS on episodic LTM have not yet been systematically and comprehensively examined 
(Antonenko et al. 2016). Recent studies on declarative LTM mainly focused on the application of tACS during sleep but there is a notable paucity of well-controlled studies specifically investigating stimulatory effects in healthy participants in an alert state (Marshall et al. 2011; Antonenko et al. 2013; Eggert et al. 2013; Prehn-Kristensen et al. 2014). The aim of this work was to identify the causal role of brain dynamics in the encoding of episodic memory in humans. How oscillations are involved in facial processing and what neocortical area is particularly crucial for this process still forms an area of open research. Even though there is already a large amount of literature, most studies only found correlative relation between brain oscillations and memory encoding.

Our research objective was firstly to define a specific neocortical area and oscillatory pattern highly connected to facial LTM encoding processes and secondly to manipulate this area by using tACS in the notified frequency to improve the encoding of faces into episodic memory. We developed two main hypotheses: (i) At first, we assumed that oscillatory dynamics in the neocortex during facial memory encoding reflect the subsequent memory effect. Accordingly, the first experiment included the recording of EEG during facial encoding focusing on the pivotal oscillatory dynamics in the neocortex. We hypothesized, that this experimental stage will reveal a certain neocortical area and a specific oscillatory frequency with a significant correlation to successfully encoded and subsequently remembered faces. Moreover, we suspected the detected brain area and frequency concerning LTM encoding to differ from EEG data during the STM task and eventually to be unique for LTM encoding processes.

The results of this work should not only stay at a descriptive level of correlation between certain brain oscillations over a defined neocortical area and LTM encoding but also demonstrate a causal link between them. Hence, we secondly hypothesized that (ii) online tACS in the priorly detected frequency over the neocortical target region will alter the oscillatory dynamics during facial memory encoding and improve LTM performance. Accordingly, we will use the parameters concerning the neocortical area and the stimulatory frequency that we derived from the EEG experiment. The induction of behavioral changes by the application of model-driven, multi-electrode tACS in the second experiment will manifest in changes in accuracy ratio and confidence during the recognition part of the task. Finally, we hypothesized the effect of tACS applied with the parameters, that were derived from the EEG experiment, to be exclusively unique for an improvement of the LTM encoding and will not have an effect on STM. In that regard, we also tested the memory retrieval after a short time interval to assess behavioral changes for STM performance and 
reaction times. If the parameters of target region and stimulation frequency are exclusive, there will not occur notable alterations in the accuracy and reaction time of the STM recognition task.

Lastly, since there is still little knowledge about tACS as a relatively recent method, this thesis shall provide further insights and deeper understanding particularly concerning its manipulative influence on memory formation. Previous studies using tACS mainly focused on motor functions, while manipulation with cognitive functions reached increasing interest only recently. However, there is still a lack of knowledge about the mechanisms behind the encoding of faces into episodic memory. In a broader sense, this study takes advantages of tACS to reveal mechanisms behind memory formation and the induction of neuroplasticity. Eventually, the experimental results might get us closer to gain knowledge about physiological structures behind episodic LTM and neural circuits in the brain and - in the long-term - enable the transfer of findings in healthy human adults to diseased patients to be a guideline for the further development of diagnostic methods and therapeutic strategies. 


\section{Methods}

\section{$2.1 \quad$ Subjects}

The study was conducted in the Department of Clinical Neurophysiology, University Medical Center Göttingen, Germany. All studies together included 60 healthy participants, who were recruited through the university job market. The participation was reimbursed at the rate of 8.5 euro per hour. All volunteers had normal or corrected to normal vision, and met the following inclusion criteria: non-smoker, no history of any psychiatric illnesses or neurological disorders, no symptoms of migraine, no epileptic history, no previous events of traumatic head injury, no substance abuse, no chronic medications (exception: oral contraceptives), no participation in another study (in parallel or in the previous two weeks), no metal implants including drug pumps, pace makers and hearing aids and no pregnancy or breast feeding.

Prior to the participation each subject was informed about the exclusion criteria, the task description, data storage policy, and possible side effects. All experiments were approved by the Ethics Committee of the University Medical Center Göttingen (ethics number 24/9/16) and were conducted in accordance with the Declaration of Helsinki.

\subsubsection{Experimental design}

The project included three studies with separate recruitment calls (see Figure 7). At first, three rounds of pilot experiments with 5 participants each were conducted in order to optimize the task and determine the parameters for the task: the delay between the Encoding and the LTM Retrieval Part and the span of face-money combinations during the Encoding Part. Altogether 15 subjects were recruited ( 7 female, 15 right-handed, mean age: $24.2 \pm 2.8$, mean of years of education: $17.1 \pm 2.1$, BMI: $21.9 \pm 2.7)$. Secondly, the EEG experiment was conducted on 20 participants (12 female, 18 right-handed, mean age: $24.3 \pm 3.1$, mean of years of education: $17.2 \pm 3.5, \mathrm{BMI}: 22.5 \pm 2.6)$. The final experimental stage - the tACS study - was based on the results of the EEG experiment, and included 25 subjects (13 female, 21 right-handed, mean age: $23.3 \pm 2.4$, mean of years of education: $16.6 \pm 1.9$, BMI: $23.3 \pm$ 3.8). The sample size was calculated using a paired, non-parametric Wilcoxon test to detect an effect size of 0.6 standards deviations or higher with $95 \%$ certainty (at alpha level $=0.05$ and beta level $=0.8$. The effect size is defined as the difference in the memory performance in the main stimulation group relative to the placebo group. 
Pilot experiment

3 rounds, 15

subjects
EEG experiment

20 subjects
tACS experiment

25 subjects

Figure 7: Organization of the project. (Own illustration.)

\subsubsection{Introductory meeting}

Each stage of the study included an introductory meeting that every subject attended prior to the participation in the experiments. Besides the organizational aspects, this was intended to make the subjects acquainted with the environment of the laboratory. All were informed verbally and in the written form about the detailed procedure of the sessions, about the methods of EEG or brain stimulation, and gave a written informed consent. All volunteers were questioned about the exclusion criteria (such as epilepsy, migraine, cardiac diseases, and metal implants), the blood pressure and pulse were measured, and all subjects passed a basic neurological checkup (correctness of pose, walk, balance, and oculomotor response). Finally, every subject was instructed about the memory tasks and trained on its demo version. They were briefed to not consume alcohol 24 hours and caffeinated drinks 4 hours before the experimental session.

\subsection{Memory test}

The subject was seated on a comfortable chair with a headrest at a table directed to the screen in front of a white wall. The touchpad as well as the mouse could be handled easily by laying both arms on the table. The distance between the subject's eyes and the screen was approximately 50 centimeters. The stimuli were displayed on the 24 ' monitor. The memory task was divided into 3 blocs: (1) an Encoding and Short-Term Memory Retrieval Part, (2) a delay of 20 minutes, (3) and a Long-Term Memory Retrieval Part. The structure of the whole memory task is shown in Figure 8. The PsychoPy software was used to implement the test (Peirce 2007). 


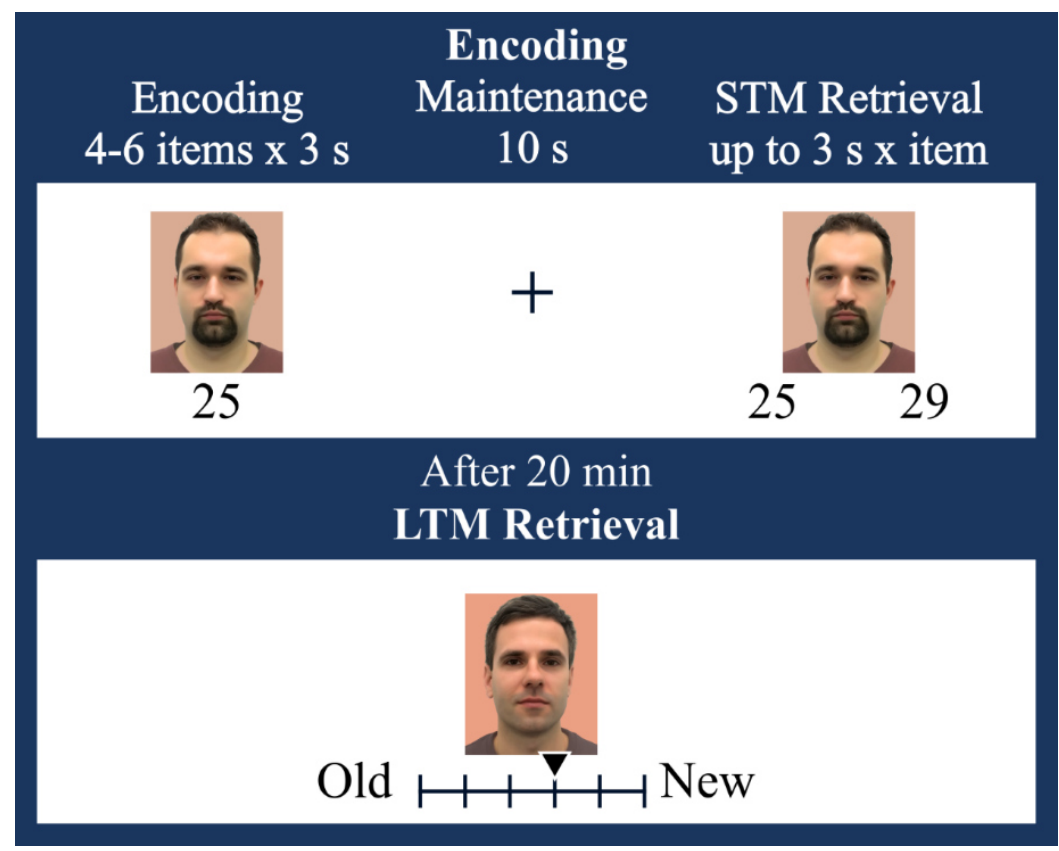

Figure 8: Memory task. Encoding and Short-Term Memory (STM) Retrieval: At first, 4 to 6 items (facemoney combinations) were displayed, each for 3 seconds. After a short maintenance of 10 seconds, all faces were shown again one by one in a pseudo-randomized order. All volunteers were asked to decide between 2 amounts of money presented below each face in a two-alternative forced-choice task. Long-Term Memory (LTM) Retrieval: For each displayed face, participants were asked to indicate whether the face was presented in the Encoding Part (Old) or not (New) in a 6-point Likert Scale. (Own illustration. The photo images contained in this figure are taken by Dr. Ivan Alekseichuk and are distributed under the Creative Common license (CC-BY-NC-ND 4.0). The photos are reused with permission.)

The Encoding and STM Retrieval block consisted of an encoding, a maintenance, and a recognition part. The volunteers needed to memorize two linked stimuli: the facial item (adult, Caucasian, male or female), and the numeric item (money value). Using pairs of facial and numerical stimuli instead of solely faces as memory items resulted from the fact, that the amount of necessary faces as lure items to present to reach sufficient difficulty in the STM task would have interfered with the performance in the LTM Retrieval Part. The facial items were taken from the gallery "Humanae" by Angelica Dass (www.angelicadass.com). The span - summarizing the number of face-money-combinations in one encoding block - was established in the first pilot experiment with 5 participants (see Figure 9). We aimed to allocate the participants' performance between $70 \%$ to $80 \%$ to neither set accuracy to chance level nor to set it too high to still allow optimization using brain stimulation. Altogether there were 90 trials. 


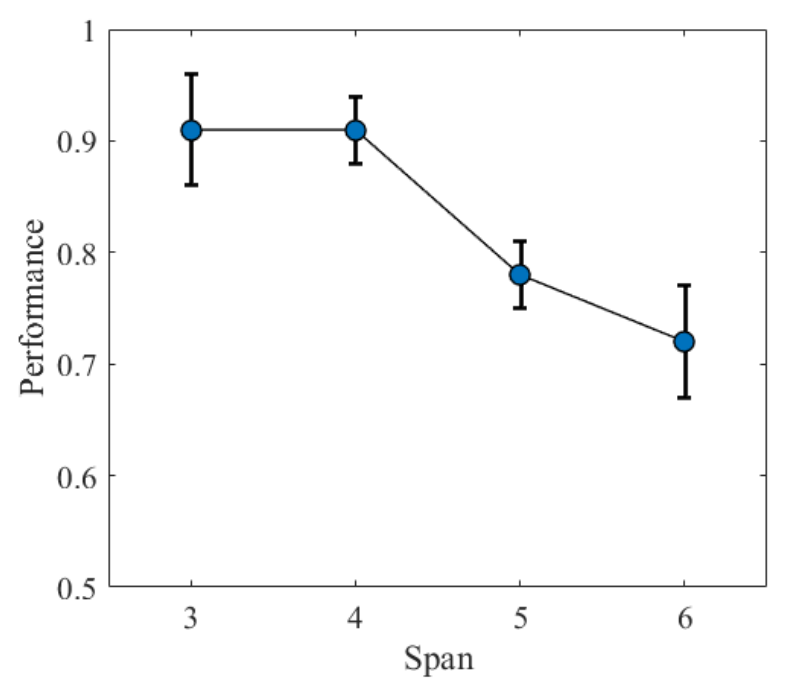

Figure 9: Performance in the first pilot experiment during the Short-Term Memory Retrieval Part as the function of the span. The pilot experiment included 5 participants. The $\mathrm{x}$-axis displays the span representing the number of face-money combinations per task block in a range from 3 to 6 . The y-axis shows the proportionate performance from 0.5 to 1 in decimal fractions calculated by the hit rate minus false alarm rate, when 1 is equivalent to all answers are correct. Data are shown as mean \pm standard error of the mean (SEM). The graphic shows a decline in performance in the range from 4 to 6. (Own illustration.)

After the encoding and short (10 s) maintenance, subjects were required to attend the testing block, which was organized as a two-alternative forced-choice task. The facial items were presented again in a pseudo-randomized order, this time with two different numeric items displayed below. The participants were asked to press the left or the right button on the response pad (RB-740, Cedurus, USA) with the respective index finger to indicate the correct numeric item. The response period lasted for 3 seconds. All subjects were asked to decide as accurately and fast as possible. The whole task block took approximately 20 minutes to complete.

Then the long delay was introduced. Its time length was established in the second and third pilot experiment with 5 participants each and was set to 20 minutes. While in the second pilot experiment the LTM Retrieval Part started after a delay of 20 minutes and resulted in a performance of $72 \%$, performance declined to $50 \%$ in a third pilot experiment equivalent to chance level when the delay was extended to $24 \mathrm{~h}$ (see Figure 10). The subjects were asked to stay in the chair and relax but to not close their eyes. 


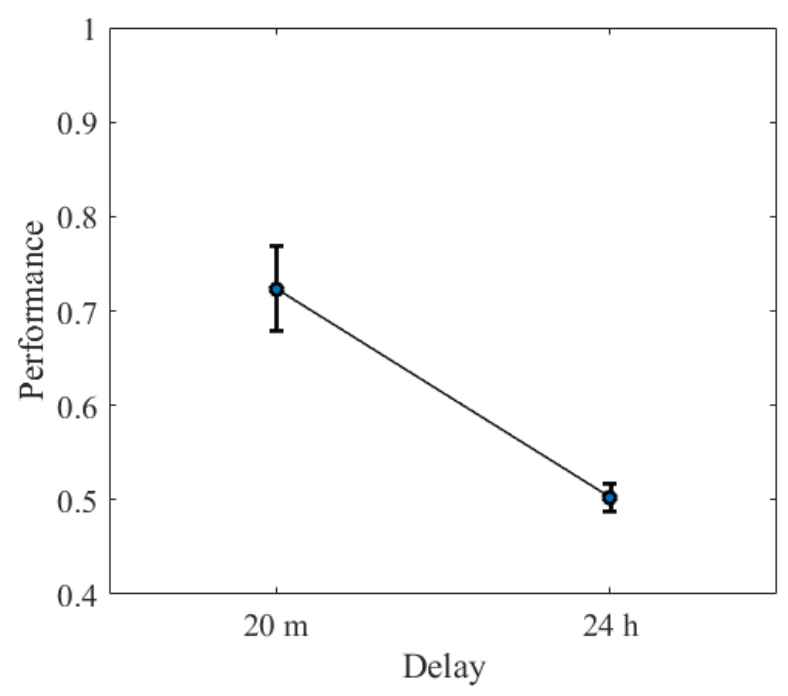

Figure 10: Performance in the second pilot experiment during the Long-Term Memory Retrieval Part as the function of the time delay between Encoding and LTM Recognition. The pilot experiment included 10 participants (5 participants with a delay of 20 minutes, 5 participants with a delay of 24 hours). The $\mathrm{x}$-axis displays the time scale with a delay of 20 minutes compared to $24 \mathrm{~h}$ in between for 5 participants each. The y-axis shows the proportionate performance from 0.4 to 1 in decimal fractions calculated by the hit rate minus false alarm rate, when 1 is equivalent to all answers are correct. Data are shown as mean \pm SEM. The performance decreases from 0.72 with a time delay of 20 minutes to 0.5 after a delay of 24 h corresponding to chance level. (Own illustration.)

The LTM Retrieval was designed in a Six-point Likert scale in an old-new recognition paradigm. Participants were requested to decide whether the face had been presented to them in the first part ("old") or not ("new"), and they were asked to indicate how certain they were about their decision by electing one of six options on a symmetrical scale ("definitely old", "probably old”, “maybe old”, “maybe new", "probably new”, and "definitely new"). A Likert scale is a psychometric scale, that is highly suitable not only to inform about the presence or absence of the item in the memory but also to detect the participant's level of confidence and range the participants' decision from low to high, while the options are balanced. We decided for a Six-point Likert scale and against a Five- or Seven-point scale to prevent participants to pick the neutral option but rather to force them to make a choice to generate more substantive data. Moreover, an improvement or deterioration in recognition does not only become visible by the various ratio of right and false answers. It is also reflected in the changing certainty visualized by the nuances of the levels of confidence that the decision is based on.

180 faces were presented one by one (90 "old" and 90 "new" faces). Participants were requested to decide whether the face had been presented to them in the first part or whether it was new, and they were asked to indicate how certain they were about their decision by 
electing one of six options: "definitely old", "probably old", "maybe old”, "maybe new", "probably new", and "definitely new". No time restriction was imposed. This task block took approximately 20 to 25 minutes to complete depending on the individual speed of each participant.

\subsection{Electroencephalography (EEG)}

\subsubsection{EEG recording}

During the task an EEG was recorded with the active amplifier (Brain Vision ActiChamp 128, Munich, Germany) from 128 scalp positions according to the International 10-05 EEG system. All recordings were referenced to FCz. According to the individual head size, a corresponding elastic electrode cap (ActiCAP, Munich, Germany) with the mounted electrodes was located on the head, and electrode gel (HighViscosity Electrolyte-Gel Super Visc, Munich, Germany) was applied between each electrode and the scalp to ensure the electrodes' conductance. Impedance was checked before every measurement and was kept below $20 \mathrm{kOhm}$. The sampling rate was set to $2 \mathrm{kHz}$. All data were recorded from DC to Nyquist frequency. EEG was recorded during the whole memory task with trigger marked memory encoding.

\subsubsection{EEG analysis}

The analysis was performed by using the MATLAB FieldTrip Toolbox. During the preprocessing the EEG data was bandpass filtered from 0.2 to $45 \mathrm{~Hz}$ and split into the epochs around the timepoint of memory encoding. After detrending the epochs, the data was downsampled to $500 \mathrm{~Hz}$. The data was visually inspected utilizing a semi-automatic routine showing the preprocessed data and removing noisy trials related to physiological artifacts such as excessive amount of muscle activity. Trials with a maximum amplitude three times higher than the average maximum amplitude among all trials were excluded. Moreover, disturbed channels were interpolated to reduce the amount of variance in the data. Finally, the EEG signals were re-referenced to the common average and the independent component analysis (ICA) was used to clean the data from the ocular artifacts (one or two rejected components per dataset).

Then, the power analysis was performed. The data was normalized and epoched according to the time interval of interest: from $0 \mathrm{~s}$ to $1 \mathrm{~s}$ after the presentation of a memory item during the encoding stage (to $0.5 \mathrm{~s}$ for the STM analysis). Then the single tapper method (Slepian 
window function) was used to estimate the power of oscillations from 1 to $45 \mathrm{~Hz}$. The trials were sorted on two categories - the subsequently recognized and unrecognized items separately according to the STM and LTM retrieval. The trials were averaged per category and subjected to the non-parametric, cluster-based permutation test (two tailed $\mathrm{p}_{\text {cor }} \leq 0.05$ ). The corresponding topoplots were generated and are reported in the Results.

\subsection{Transcranial alternating current stimulation (tACS)}

During the brain stimulation study, tACS was used to manipulate with the oscillation of interest (Antal et al. 2008). This study was a double-blinded, randomized, cross-over and placebo-controlled investigation. Three different stimulation conditions were introduced based on the results of the EEG experiment: at first the target stimulation with applying tACS on the right hemisphere, secondly the active control condition with applying tACS on the left hemisphere and finally the placebo condition with the application of sham stimulation in a fade-in/fade-out protocol. The sham stimulation ensured the placebocontrolled design. Neither the participants nor the investigator knew whether during a session it was applied sham stimulation or tACS, therefore the conditions were coded and labeled as alphabetic letters in the stimulation device and the randomization table. All participants completed the three stimulation conditions in a counterbalanced order and were allocated randomly to the sequence of conditions. The randomization was implemented using the MATLAB function randperm.m. Between two sessions we set a minimum distance of three nights between to diminish carry-over effects. At the beginning of every session, the scalp location for the electrodes were marked according to the 10-20 system, and the skin was disinfected with alcohol-based skin antiseptic (Kodan). A conductive cream (Ten20 paste, Weaver and Company, Aurora, USA) was applied at the scalp to fix five round rubber electrodes $(\mathrm{r}=1 \mathrm{~cm})$ and reduce the impedance caused by the skin. The stimulation was delivered via the StarStim stimulator (Neuroelectrics, Barcelona, Spain), and controlled by NIC 2 software (same company). At the end of every session, all participants filled in a standardized questionnaire (Antal et al. 2017) about their sensations during the session.

\subsubsection{Stimulation protocol}

Every participant received alternating current or sham during the Encoding and STM Retrieval task block. The current was applied in three different conditions: sham stimulation, stimulation of the right temporo-parieto-occipital (TPO) area, as this brain region was identified in the EEG experiment, and stimulation of the left TPO area. The current was 
applied with an intensity of $1.5 \mathrm{~mA}$ peak-to-baseline and with a frequency of $4 \mathrm{~Hz}$, which was identified in the EEG experiment. Current was built up for 10 seconds and lasted for 20 minutes, i.e. for the whole length of the task block. The electrodes were fixed according to the international 10-05 system and impedance always remained below $20 \mathrm{kOhm}$. The right TPO area was targeted by using the electrodes at the positions P4, C2, CP1, T8 and Oz (see Figure 12). For the control condition, the electrodes were located at the positions P3, C1, CP2, T7 and Oz (see Figure 12). The central electrode (right TPO: P4, left TPO: P3) delivered 100 percent of the electrical current, all others conducted $25 \%$ of current each. The same electrode montages were used for the sham stimulation, when the current was applied in a fade-in-fade-out manner only for 10 seconds at the beginning and at the end of the task block (see Figure 11).

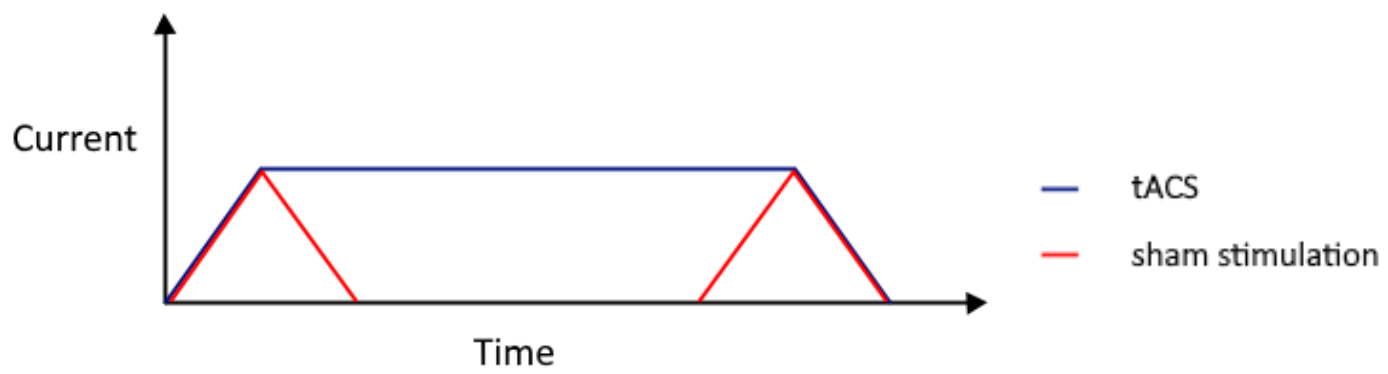

Figure 11: Current application. The application of current during the active tACS (blue) in contrast to the application during the sham stimulation (red). The $\mathrm{x}$-axis shows the time, the y-axis shows the corresponding current during the experimental procedure. In the active conditions the current was built up for 10 seconds and turned off after 20 minutes. For the sham stimulation the current was ramped up for 10 seconds and then turned off at the beginning and at the end of the task block. (Own illustration.) 

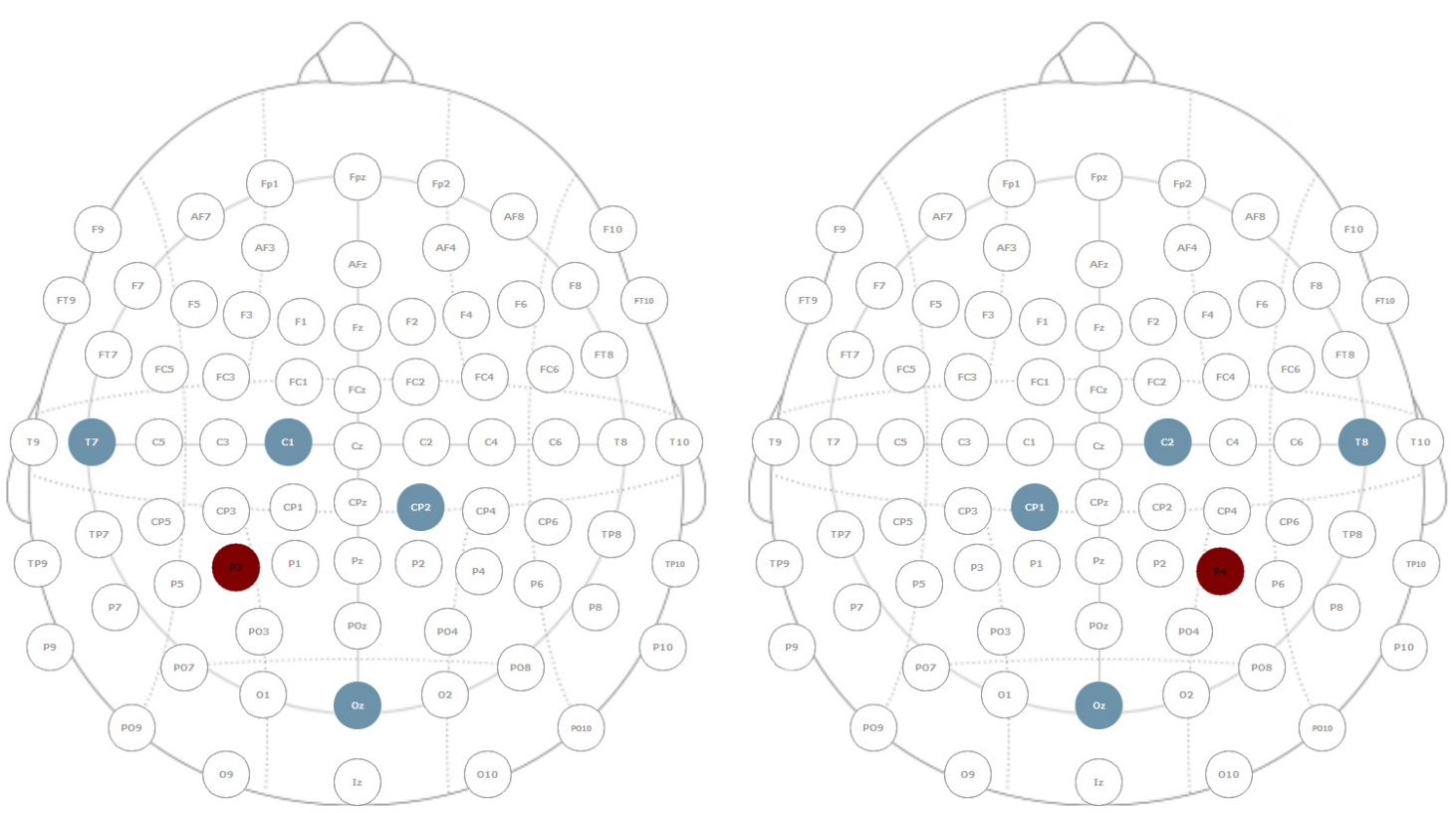

Figure 12: Electrode montage. Left: Electrode montage during the stimulation of the left hemisphere. The electrodes were positioned at P3, T7, C1, CP2 and Oz., while alternating current flows between the central electrode P3 (red) and the reference electrodes (blue). Right: Electrode montage during the stimulation of the right hemisphere. The electrodes were positioned at P4, T8, C2, CP1 and Oz, while alternating current flows between the central electrode P4 (red) and the reference electrodes (blue). The image was made using NIC software (Neuroelectrics, Spain). 


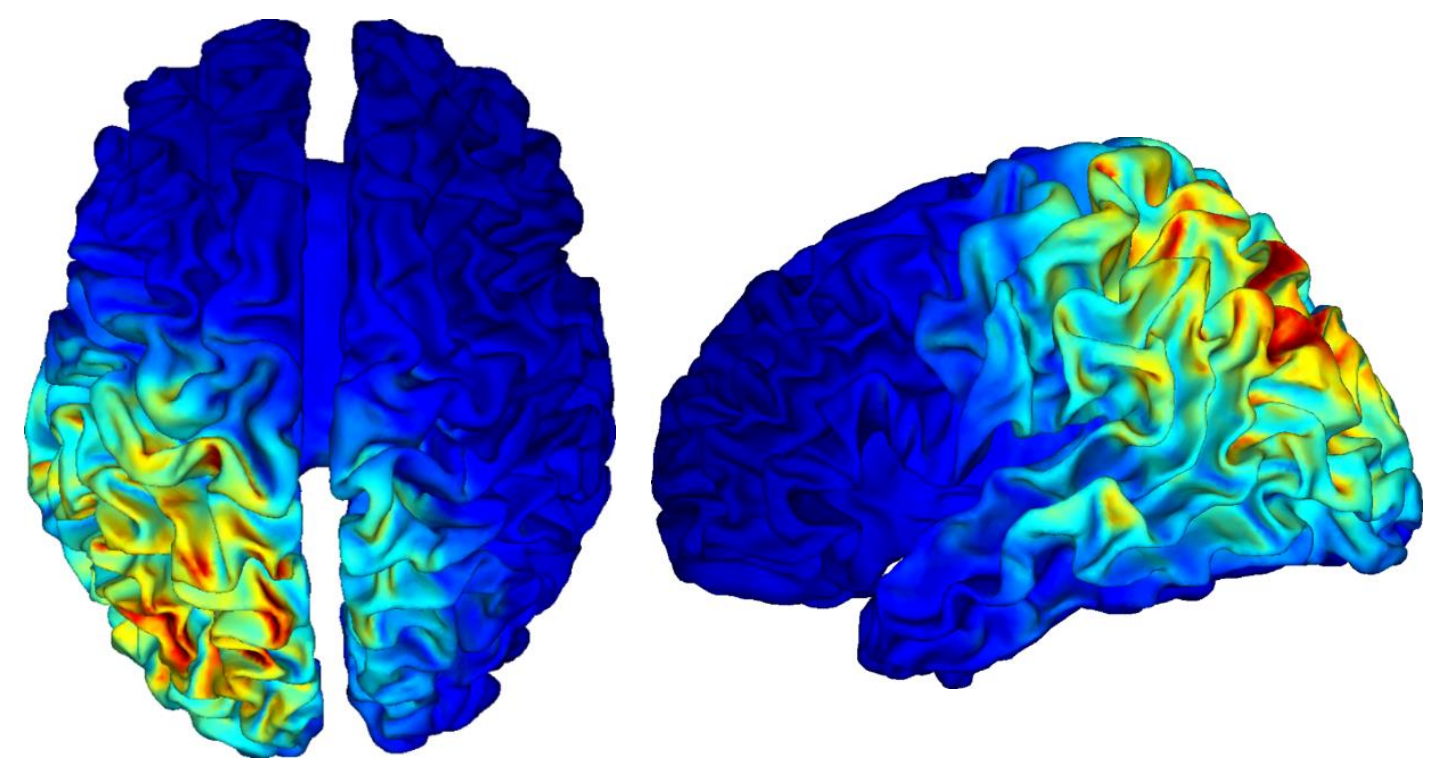

Figure 13: Simulation of the electric field during the stimulation of the left TPO area. Color-coded from 0 (dark blue) to $0.3 \mathrm{mV} / \mathrm{mm}$ (dark red). Left: shows a top view on the left hemisphere. Right: shows a side view on the left hemisphere. Both graphics show an electric field of $\geq 0.1 \mathrm{mV} / \mathrm{mm}$ in the left TPO area. The brain images were made using NIC software (Neuroelectrics, Spain).

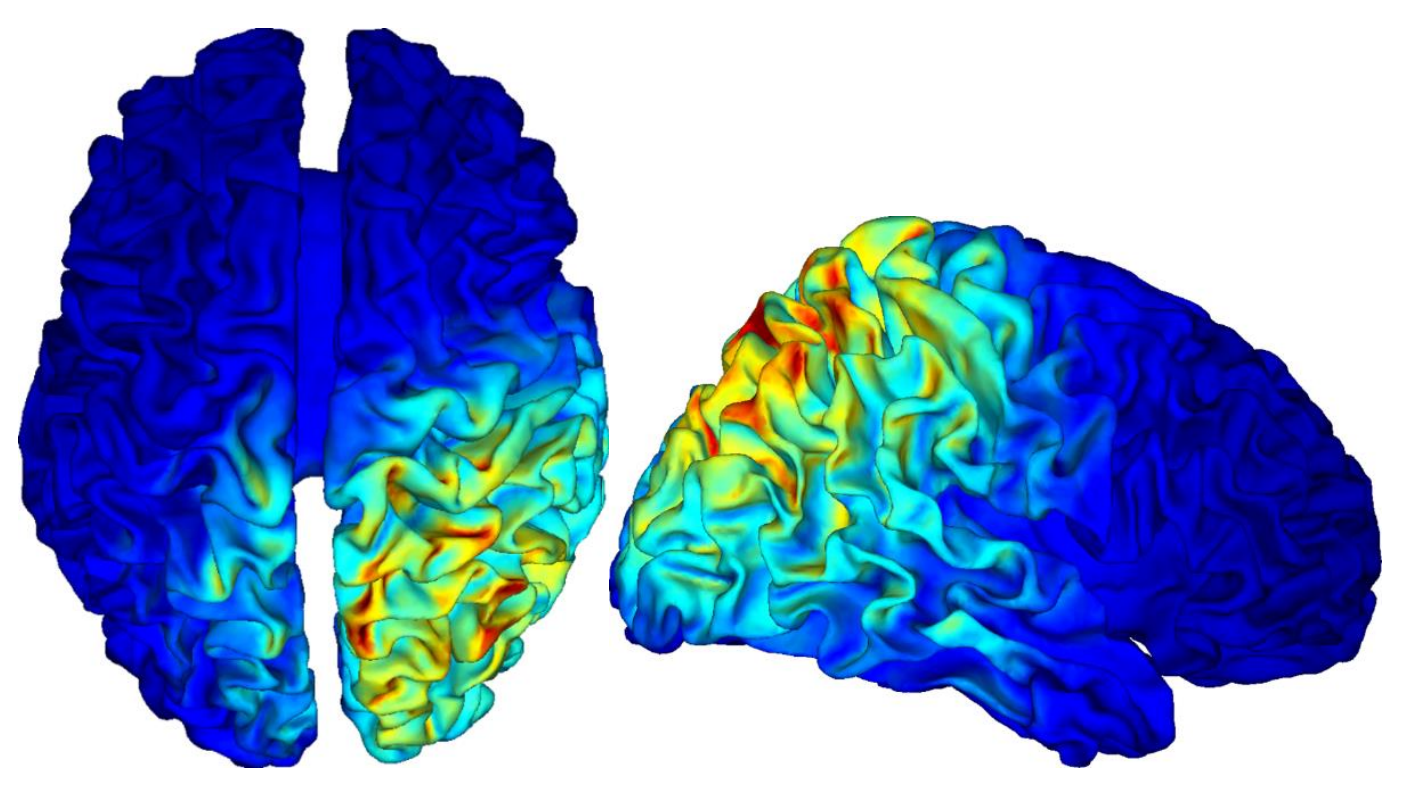

Figure 14: Simulation of the electric field during the stimulation of the right TPO area. Color-coded from 0 (dark blue) to $0.3 \mathrm{mV} / \mathrm{mm}$ (dark red). Left: shows a top view on the right hemisphere. Right: shows a side view on the right hemisphere. Both simulation models show an electric field of $\geq 0.1 \mathrm{mV} / \mathrm{mm}$ in the right TPO area. The brain images were made using NIC software (Neuroelectrics, Spain). 
The electric field induced by the corresponding montages on the left and right hemispheres were projected on the brain surface between the white and grey matter (see Figure 13 and 14). They were generated according to Miranda et al. (2013) by using the build.in head model in the NIC 2 software (Neuroelectrics). For each hemisphere the models show a maximum electric field in the TPO areas with values above the critical threshold level $(0.1 \mathrm{mV} / \mathrm{mm})$ for inducing the entrainment effects.

\subsection{Behavioral analysis}

Statistical analysis was performed with MATLAB. For the STM subtask, the performance (false alarm rates deducted from the hit rates, given in in \% of correct responses) and reaction time (in seconds) were analyzed under the active stimulation conditions relative to the sham stimulation. A two-tailed, non-parametric, paired Wilcoxon test was utilized ( $p \leq 0.05)$. All data is reported as mean and standard error of the mean (SEM). For the LTM subtask, the performance score (false alarm rates deducted from the hit rates, given in \% of correct responses) and decision confidence (in confidence points, from 1 to 3) were analyzed separately, as well as their cumulative product, the weighted performance. The weighted performance is the total score, where for every correct task choice a volunteer received +3 if the decision was very confident, +2 if it was confident, and +1 if it was not confident. For every wrong task choice, the same number of points was deducted from the total score. A two-tailed, non-parametric, paired Wilcoxon test was utilized for the statistical comparison of the performance and confidence under active stimulation vs. sham stimulation for each hemisphere $(\mathrm{p} \leq 0.05)$. All data is reported as mean and SEM. 


\section{Results}

\subsection{The EEG experiment}

\subsubsection{Behavioral results}

The EEG experiment aimed to identify the anatomical brain region where the activity during memory encoding relates to subsequently recollected answers. Every participant's individual performance was estimated by deducting the false alarm rates from the hit rates. The analysis of the Short-Term Memory Recognition Part revealed decreasing performance over all 20 participants with the increasing number of items (mean performance in $\% \pm$ standard error of the mean (SEM), 4 items: $87 \pm 2 \%, 5$ items: $80 \pm 2 \%, 6$ items: $72 \pm 3 \%$ ) with a mean performance of $80 \pm 2 \%$ as total average. The opposite trend appeared for the reaction times (mean reaction time in seconds \pm SEM, 4 items: $1.34 \pm 0.07 \mathrm{~s}, 5$ items: $1.39 \pm 0.07 \mathrm{~s}, 6$ items: $1.48 \pm 0.07 \mathrm{~s}$ ) providing a mean reaction time of $1.4 \pm 0.07 \mathrm{~s}$ SEM.

Further, the accuracy of responses in the Long-Term Memory Part was analyzed. The results showed a mean accuracy of $77 \pm 2 \%$ SEM with a mean level of confidence of $2.18 \pm$ 0.09 SEM (where 1 is the lowest and 3 is the highest level of confidence).

\subsubsection{EEG results}

A non-parametric, cluster-based permutation test (two tailed $\mathrm{p}_{\mathrm{cor}} \leq 0.05$ ) was employed to investigate the significant differences in the brain activity recorded in the EEG for 20 participants during memory encoding in comparison to the baseline using the EEG data with one second periods immediately before the presentation of the memory item. The analysis was performed in the sensor-space - referring to a depiction of EEG data at the stage of the original sensors without further transformations - for all 128 channels (10-05 electrode placement system). The full EEG spectrum from 1 to $45 \mathrm{~Hz}$ was analyzed.

\subsubsection{Short-Term Memory Part}

The topographic maps (see Figure 15-17) show the brain activity during the Encoding Part of STM. For subsequently forgotten items in the Short-Term Memory Part it could be observed an increase in low theta power (mean power $4 \pm 1 \mathrm{~Hz}$ SEM) in the posterior areas of the neocortex (see Figure 15). In contrast, Figure 16 shows the brain activity for correctly recognized memory items. In addition to the posterior cluster, the medial frontal cluster demonstrates an increase in low theta activity. The difference between the remembered and 
forgotten trials highlights a major increase in power, i.e. in local synchronization of brain activity in the frontal cortex in the low theta range (see Figure 17).

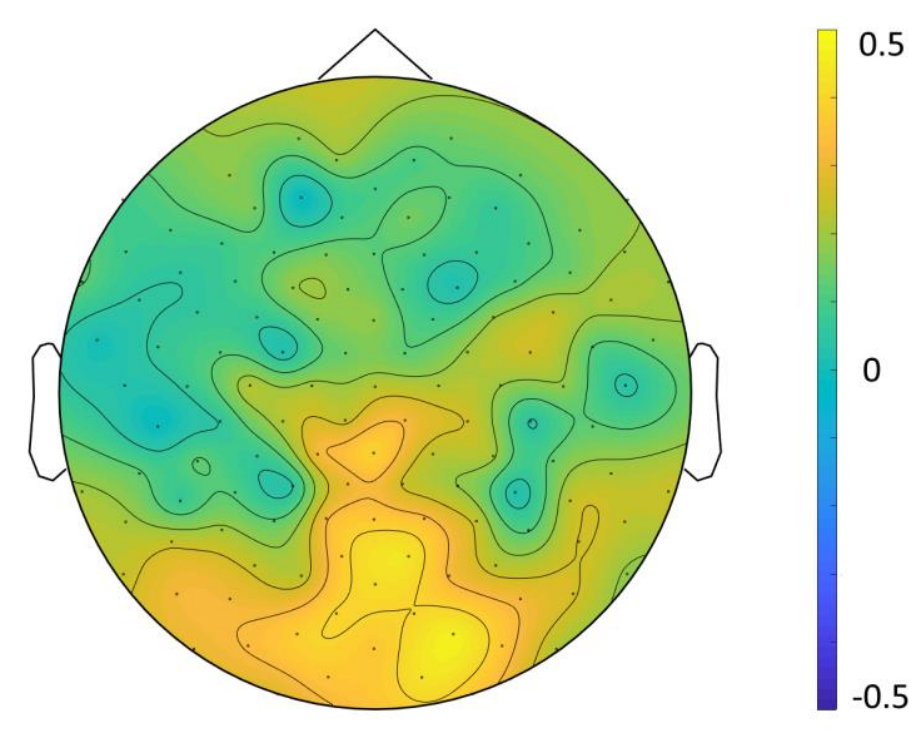

Figure 15: Topographic map of the human brain during memory encoding for subsequently forgotten items in the Short-Term Memory Task. (EEG experiment.) The map includes the data of 20 participants. The brain synchronization is color-coded in relative units from - 0.5 in dark blue representing desynchronization to 0.5 in yellow depicting synchronization. The frequency analysis reveals an increase in low theta power in the posterior cortex. (Own illustration.) 


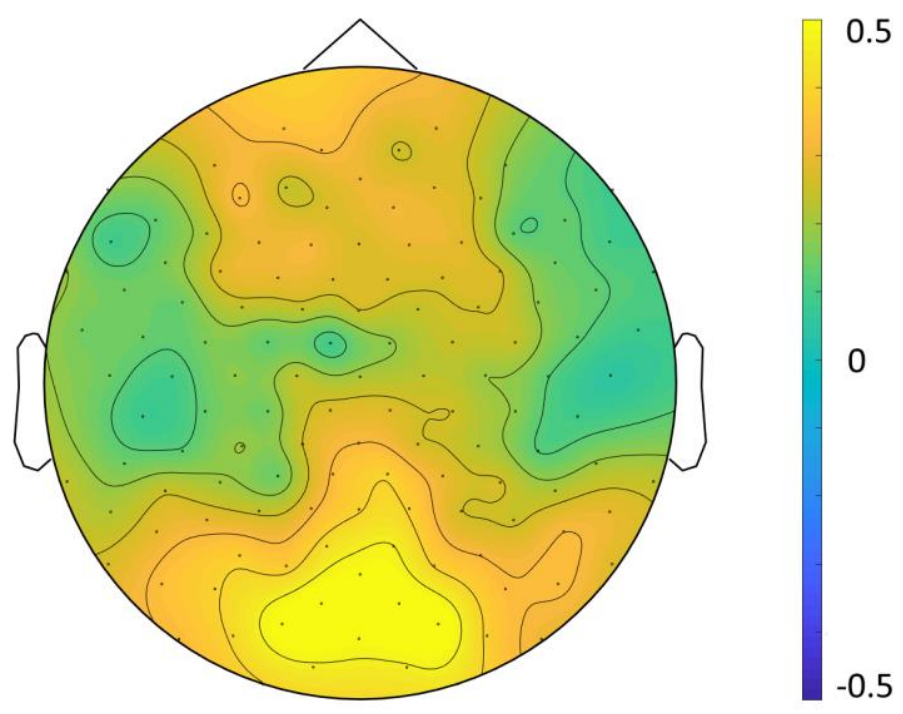

Figure 16: Topographic map of the human brain during memory encoding for subsequently remembered items in the Short-Term Memory Task. (EEG experiment.) The map includes the data of 20 participants. The brain synchronization is color-coded in relative units from -0.5 in dark blue representing desynchronization to 0.5 in yellow depicting synchronization. The frequency analysis reveals an increase in low theta power in the posterior and the frontal cortex. (Own illustration.)

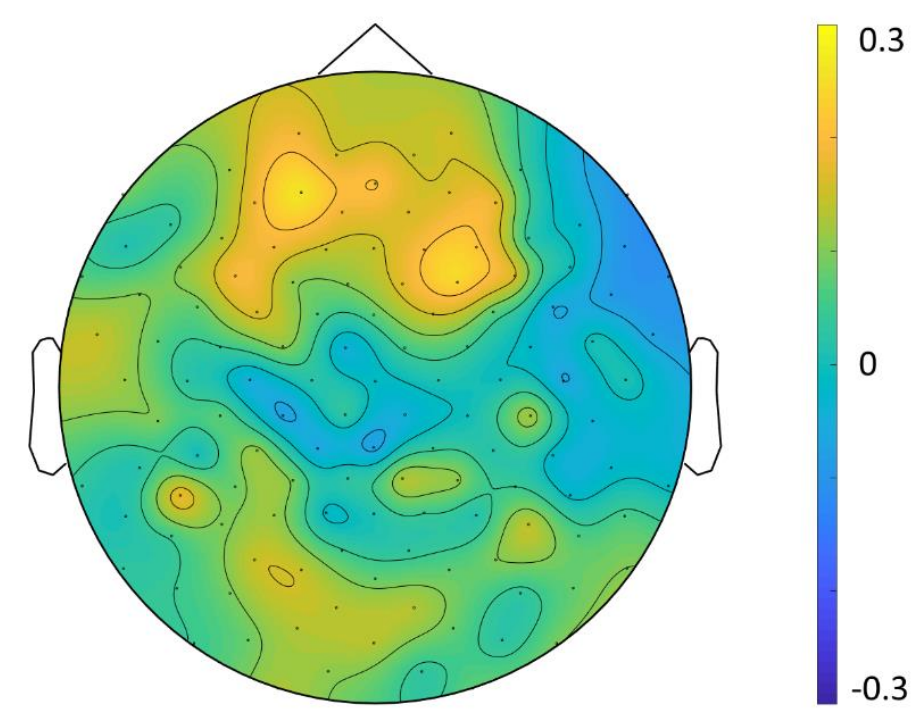

Figure 17: Difference in brain activity during encoding between subsequently remembered and forgotten answers in the Short-Term Memory Task. (EEG experiment.) The map includes the data of 20 participants. The brain synchronization is color-coded in relative units from -0.3 in dark blue representing desynchronization to 0.3 in yellow depicting synchronization. The arithmetic difference of the frequency analysis between brain activity during remembered and forgotten trials reveals a significant increase in power in the low theta range in the frontal cortex. (Own illustration.) 


\subsubsection{Long-Term Memory Part}

For the investigation of brain activity during the encoding of LTM, the trials of all 20 participants were sorted depending on the response in the Long-Term Memory Recognition Part. The analysis of the subsequently forgotten items shows a decrease in low theta power (mean power $4 \pm 1 \mathrm{~Hz} \mathrm{SEM}$ ), i.e. local desynchronization, in the left prefrontal cortex and in the right temporo-parieto-occipital cortex (see Figure 18). Interestingly, synchronization of brain activity was detected in the frontal and the posterior cortex for subsequently recognized answers (see Figure 19). Figure 20 demonstrates locations of two clusters of difference between the subsequently remembered and forgotten items in the left frontal cortex and the right temporo-parieto-occipital area. Both clusters emerge at the low theta range.

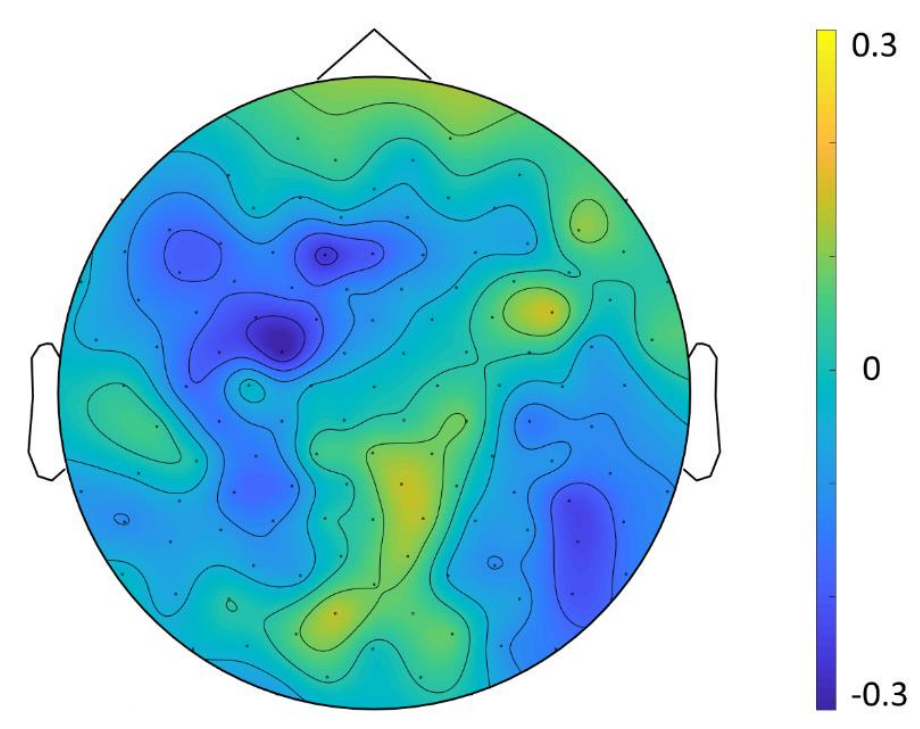

Figure 18: Topographic map of the human brain during memory encoding for subsequently forgotten items in the Long-Term Memory Task. (EEG experiment.) The map includes the data of 20 participants. The brain synchronization is color-coded in relative units from -0.3 in dark blue representing desynchronization to 0.3 in yellow depicting synchronization. The frequency analysis shows a decrease in low theta power for the left prefrontal and the right temporo-parieto-occipital (TPO) area. (Own illustration.) 


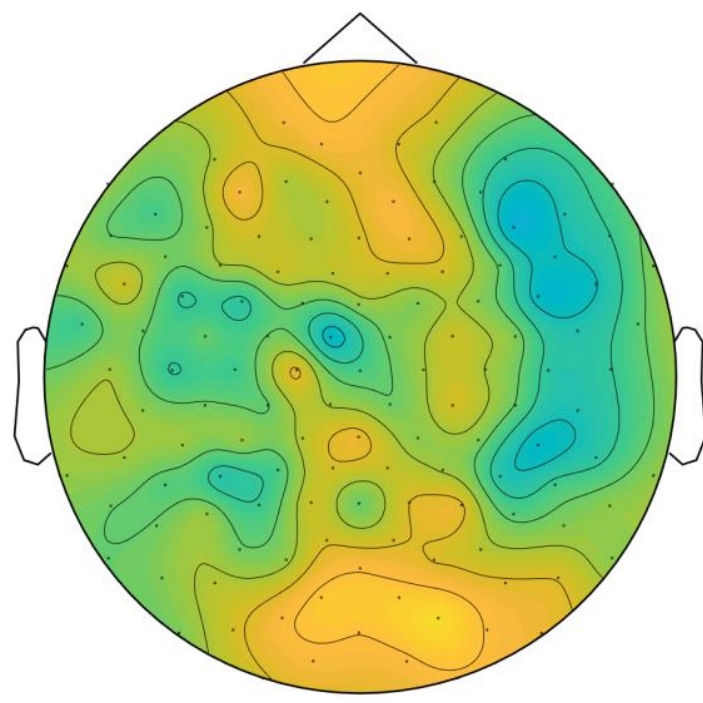

Figure 19: Topographic map of the human brain during memory encoding for subsequently remembered items in the Long-Term Memory Task. (EEG experiment.) The map includes the data of 20 participants. The brain synchronization is color-coded in relative units from -0.3 in dark blue representing desynchronization to 0.3 in yellow depicting synchronization. The frequency analysis shows an increase in low theta power in the frontal and occipital cortex. (Own illustration.)

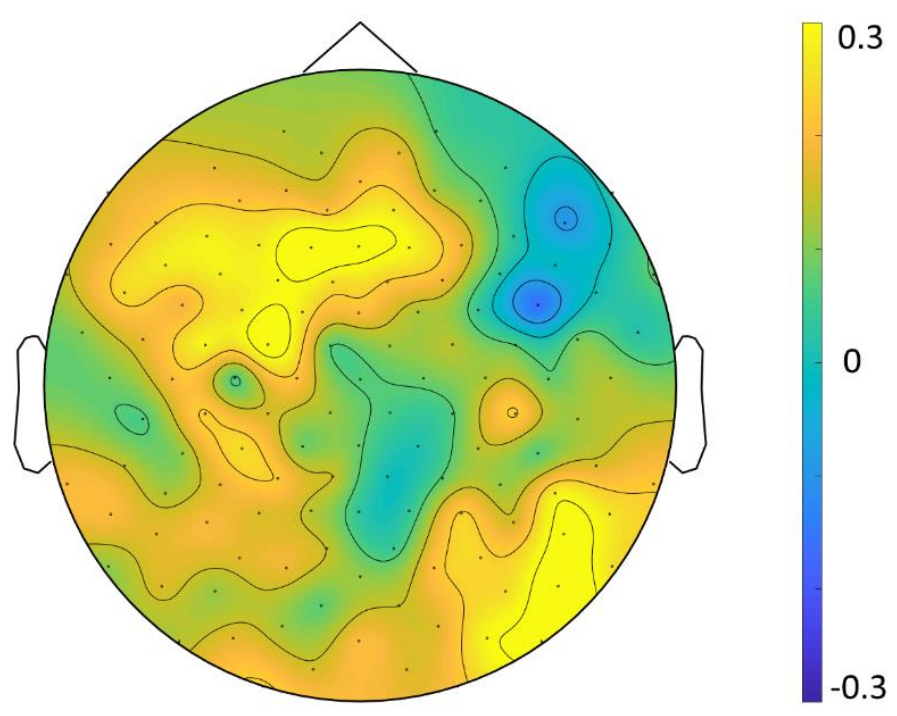

Figure 20: Difference in brain activity during encoding between subsequently remembered and forgotten answers in the Long-Term Memory Task. (EEG experiment.) The map includes the data of 20 participants. The brain synchronization is color-coded in relative units from -0.5 in dark blue representing desynchronization to 0.5 in yellow depicting synchronization. The arithmetic difference of the frequency analysis between brain activity during remembered and forgotten trials reveals a significant increase in power in the low theta range in the left prefrontal and the right TPO area. (Own illustration.) 


\subsection{The tACS experiment}

\subsubsection{Stimulation results}

To further investigate the relationships between the low theta activity and memory encoding the tACS experiment was conducted. It was designed as a double-blinded, placebocontrolled, counterbalanced, randomized, and crossover study. Three stimulation conditions were introduced: $4 \mathrm{~Hz}$ stimulation over the right TPO area, $4 \mathrm{~Hz}$ stimulation over the left TPO area, and sham stimulation. The entirety of 25 participants of this experimental stage is included in the calculations. The results of sham stimulation form the baseline and are compared with each of both tACS conditions.

\subsubsection{Short-Term Memory Part}

The purpose of this experimental stage was to stimulate the earlier identified hotspots during the memory encoding that were shown to correlate with the successful LTM performance. For analysis, the accuracy (hit rates minus false alarm rate) was calculated in percent and compared over the rising number of items. The results are displayed in Figure 21 (mean performance in $\% \pm$ SEM, 4 items: sham $84 \pm 1 \%$, tACS over left hemisphere (LH) $85 \pm$ $1 \%$, tACS over right hemisphere $(\mathrm{RH}) 86 \pm 2 \% ; 5$ items: sham $80 \pm 2 \%$, LH tACS $82 \pm 2 \%$, RH tACS $79 \pm 2 \% ; 6$ items: sham $76 \pm 2 \%$, LH tACS $77 \pm 2 \%$, RH tACS $77 \pm 2 \%$ ). As shown in Figure 22, the overall average accuracy for the Short-Term Memory Part was $80 \pm$ $2 \%$ SEM for sham condition, $81 \pm 1 \%$ SEM for the stimulation of the left hemisphere and $80 \pm 2 \%$ SEM for the stimulation of the right hemisphere. Further statistical analysis using a two-tailed, non-parametric Wilcoxon test revealed no significant differences in performance between sham stimulation and the stimulation of the left or right hemisphere (mean performance during LH tACS compared to sham stimulation for 4 items: $\mathrm{p}=0.58$, mean performance during RH tACS compared to sham stimulation for 4 items: $p=0.37$; mean performance during LH tACS compared to sham stimulation for 5 items: $p=0.29$, mean performance during RH tACS compared to sham stimulation for 5 items: $\mathrm{p}=0.38$; mean performance during LH tACS compared to sham stimulation for 6 items $p=0.85$, mean performance during RH tACS compared to sham stimulation for 6 items $\mathrm{p}=0.78$ ). The same applies for the overall averaged accuracy for all items (see Figure 22; mean performance during LH tACS compared to sham stimulation for $4-6$ items: $p=0.76$, mean performance during RH tACS compared to sham stimulation for 4-6 items: $p=0.97$ ). 


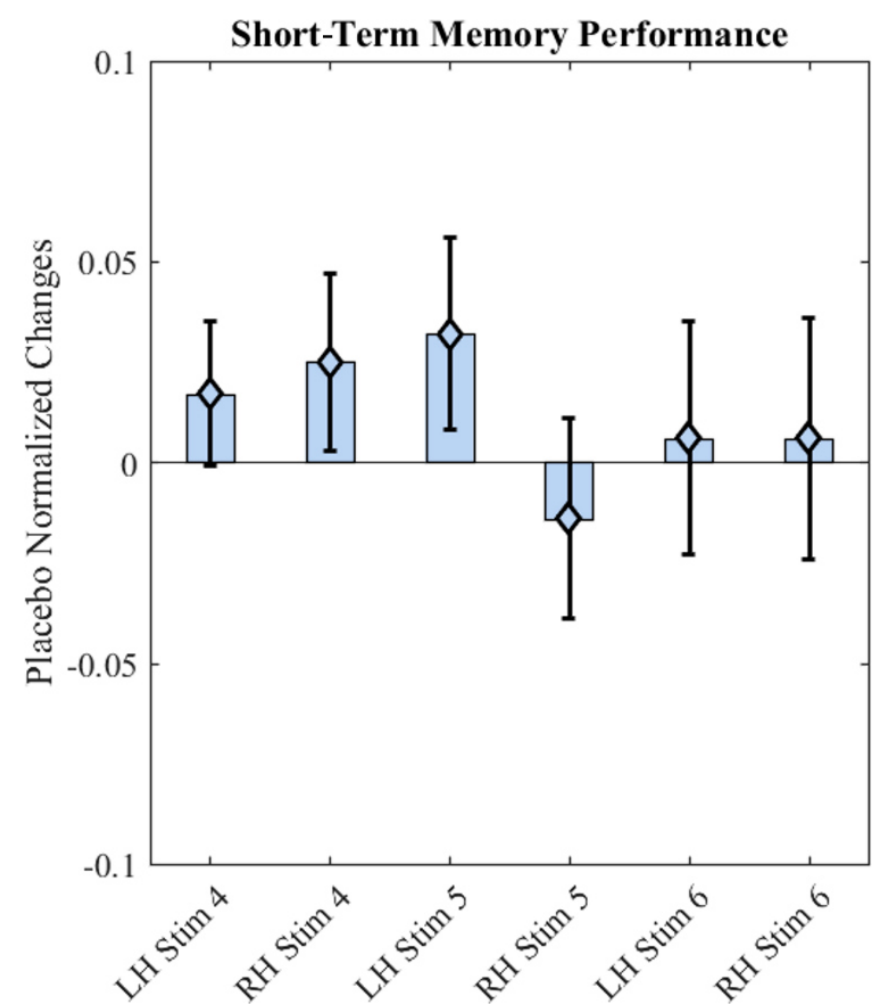

Figure 21: Placebo normalized changes of the mean performance during the Short-Term Memory Part. (tACS experiment.) The diagram includes the data of 25 participants. Placebo-normalized changes comprise the mean performance in the stimulation group relative to the placebo group and are estimated as the difference between the performance in the stimulation group and placebo group divided by the performance in the placebo group. LH Stim: tACS over left hemisphere, RH Stim: tACS over right hemisphere. The single digits on the $\mathrm{x}$-axis $(4,5$ or 6$)$ indicate the number of the memory items per single task block. Each bar displays a single stimulation condition. The placebo normalized changes are plotted as decimal fractions on the y-axis where the baseline is defined by the mean performance in the placebo group. Positive values show an increased performance, negative values show a deterioration of the performance. The error bars display the standard error of the mean (SEM). (Own illustration.) 


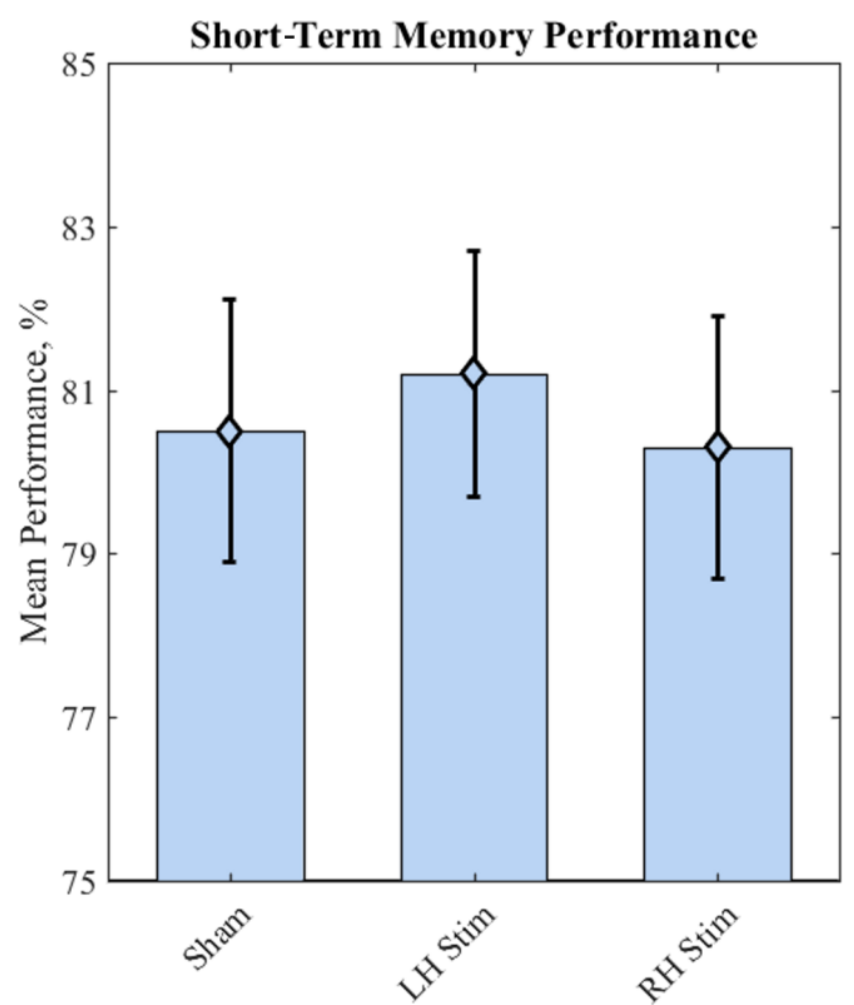

Figure 22: Mean memory performance in the Short-Term Memory Part. (tACS experiment.) The diagram comprises the data of 25 participants. Each bar plotted on the x-axis illustrates one stimulation condition: sham stimulation (Sham), stimulation of the left hemisphere (LH Stim) and stimulation of the right hemisphere (RH Stim). The y-axis indicates the mean performance in percent. The error bars display the SEM. (Own illustration.)

Also, reaction times show no significant differences (mean reaction time in seconds \pm SEM, 4 items: sham $1.31 \pm 0.05 \mathrm{~s}, \mathrm{LH}$ tACS $1.32 \pm 0.05 \mathrm{~s}$, RH tACS $1.28 \pm 0.04 \mathrm{~s} ; 5$ items: sham $1.38 \pm 0.04 \mathrm{~s}, \mathrm{LH}$ tACS $1.40 \pm 0.04 \mathrm{~s}, \mathrm{RH}$ tACS $1.35 \pm 0.04 \mathrm{~s} ; 6$ items: sham $1.42 \pm 0.04 \mathrm{~s}, \mathrm{LH}$ tACS $1.43 \pm 0.04 \mathrm{~s}, \mathrm{RH}$ tACS $1.38 \pm 0.04 \mathrm{~s}$; see Figure 23). For all trials, the mean reaction time approached $1.37 \pm 0.04 \mathrm{~s}$ SEM for sham stimulation, $1.38 \pm 0.04 \mathrm{~s}$ SEM for the stimulation of the left hemisphere and $1.34 \pm 0.04 \mathrm{~s}$ SEM for the stimulation of the right hemisphere (see Figure 24). The two-tailed, non-parametric Wilcoxon test indicates no statistically significant changes due to the stimulation (4 items: mean reaction time during LH tACS compared to sham stimulation $\mathrm{p}=0.89$, mean reaction time during RH tACS compared to sham stimulation $\mathrm{p}=0.55,5$ items: mean reaction time during LH tACS compared to sham stimulation $\mathrm{p}=0.83$, mean reaction time during RH tACS compared to sham stimulation $\mathrm{p}=0.31,6$ items: mean reaction time during LH tACS compared to sham stimulation $\mathrm{p}=0.99$, mean reaction time during RH tACS compared to sham stimulation $\mathrm{p}=0.43$ ). Consequently, there were no significant differences with respect to the mean reaction time for overall trials (mean reaction time during LH tACS compared to sham 
stimulation for 4-6 items: $\mathrm{p}=0.73$, mean reaction time during $\mathrm{RH}$ tACS compared to sham stimulation for 4-6 items: $\mathrm{p}=0.32$.

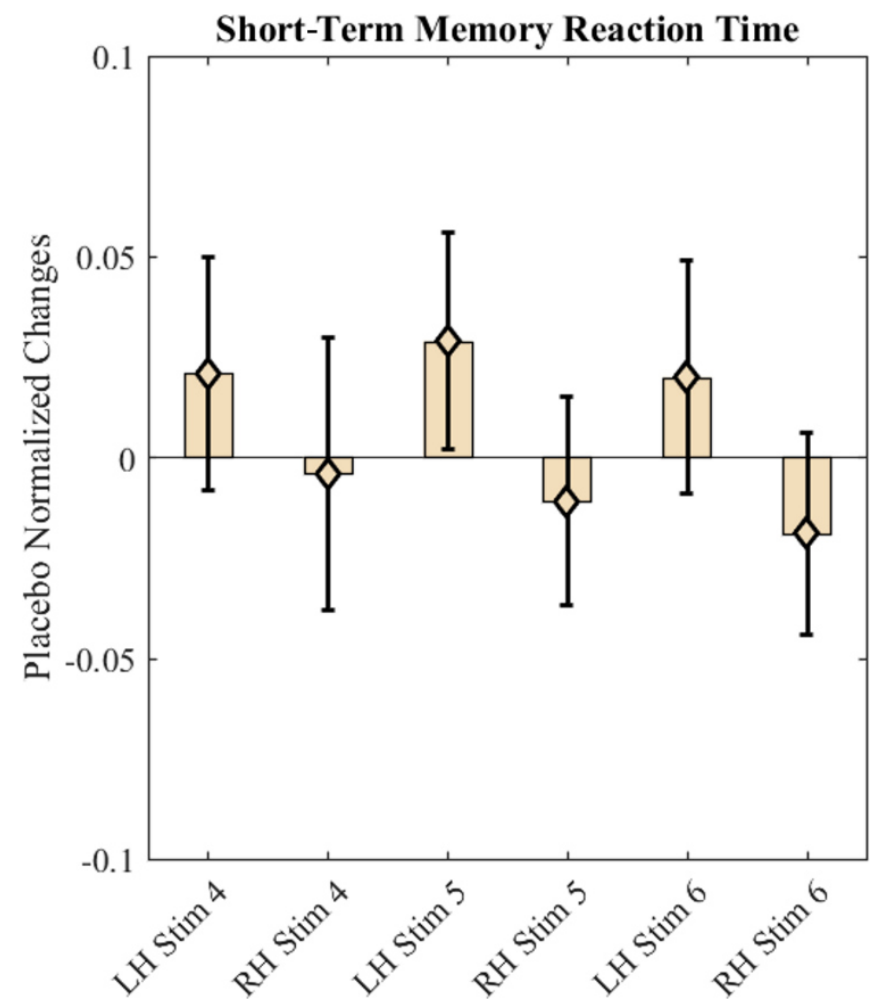

Figure 23: Placebo normalized changes of the mean reaction time during the Short-Term Memory

Part. (tACS experiment.) The diagram includes the data of 25 participants. Placebo-normalized changes comprise the mean performance in the stimulation group relative to the placebo group and are estimated as the difference between the performance in the stimulation group and placebo group divided by the performance in the placebo group. LH Stim: tACS over left hemisphere, RH Stim: tACS over right hemisphere. The single digits on the $\mathrm{x}$-axis $(4,5$ or 6$)$ indicate the number of the memory items per single task block. Each bar displays a single stimulation condition. The placebo normalized changes are plotted as decimal fractions on the y-axis where the baseline is defined by the mean performance in the placebo group. Positive values show an increased performance, negative values show a deterioration of the performance. The error bars display the SEM. (Own illustration.) 


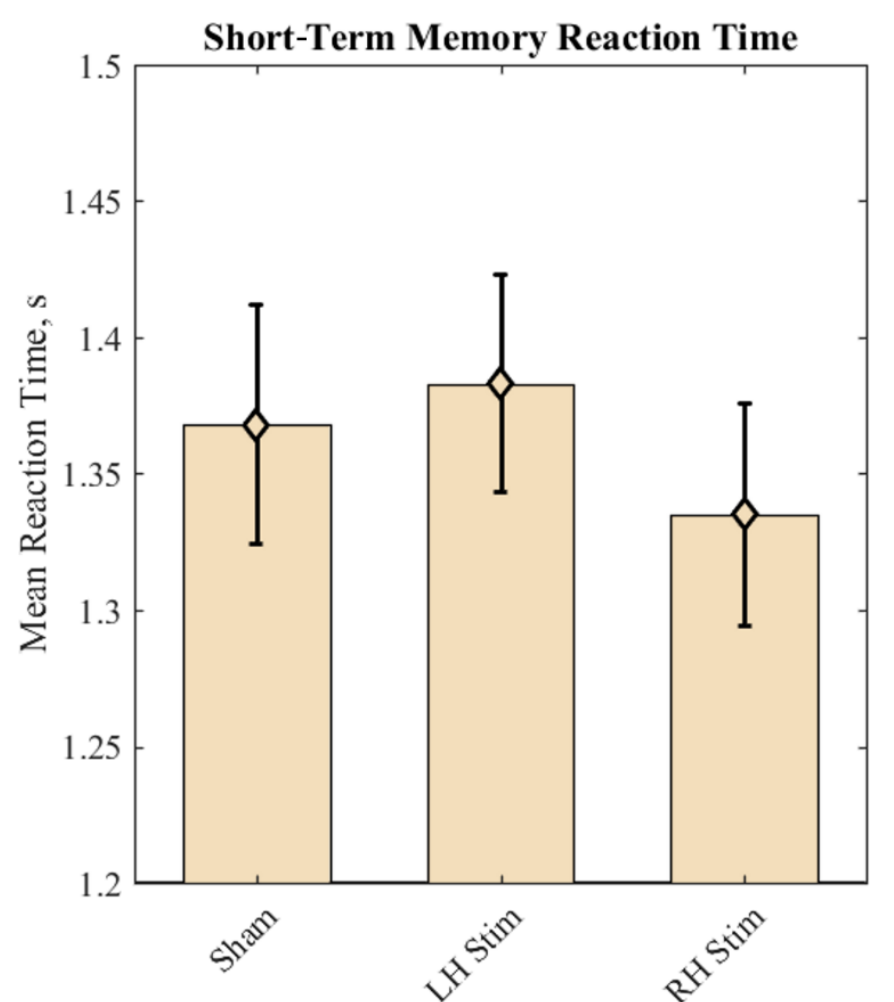

Figure 24: Mean reaction time in the Short-Term Memory Part. (tACS experiment.) The diagram comprises the data of 25 participants. Each bar plotted on the x-axis illustrates one stimulation condition: sham stimulation (Sham), stimulation of the left hemisphere (LH Stim) and stimulation of the right hemisphere (RH Stim). The y-axis indicates the mean reaction time in seconds. The error bars display the SEM. (Own illustration.)

\subsubsection{Long-Term Memory Part}

For the analysis of the Long-Term Memory Part we evaluated the performance, confidence levels and weighted performance. The confidence levels (1 - "maybe", 2 - "probably", 3 - "definitely") reflect the participant's certainty regarding the recognition of faces. Figure 25 shows the mean confidence levels for the corresponding stimulation condition with a mean confidence level of $2.11 \pm 0.06$ SEM for sham stimulation, a mean confidence level of $2.14 \pm 0.06$ SEM for the stimulation of the left hemisphere and a mean confidence level of $2.13 \pm 0.07$ SEM for the stimulation of the right hemisphere. For stimulation, confidence increased numerically but did not reach statistical significance (mean confidence level during LH tACS compared to sham stimulation $\mathrm{p}=0.24$, mean confidence level during RH tACS compared to sham stimulation $\mathrm{p}=0.59)$. 


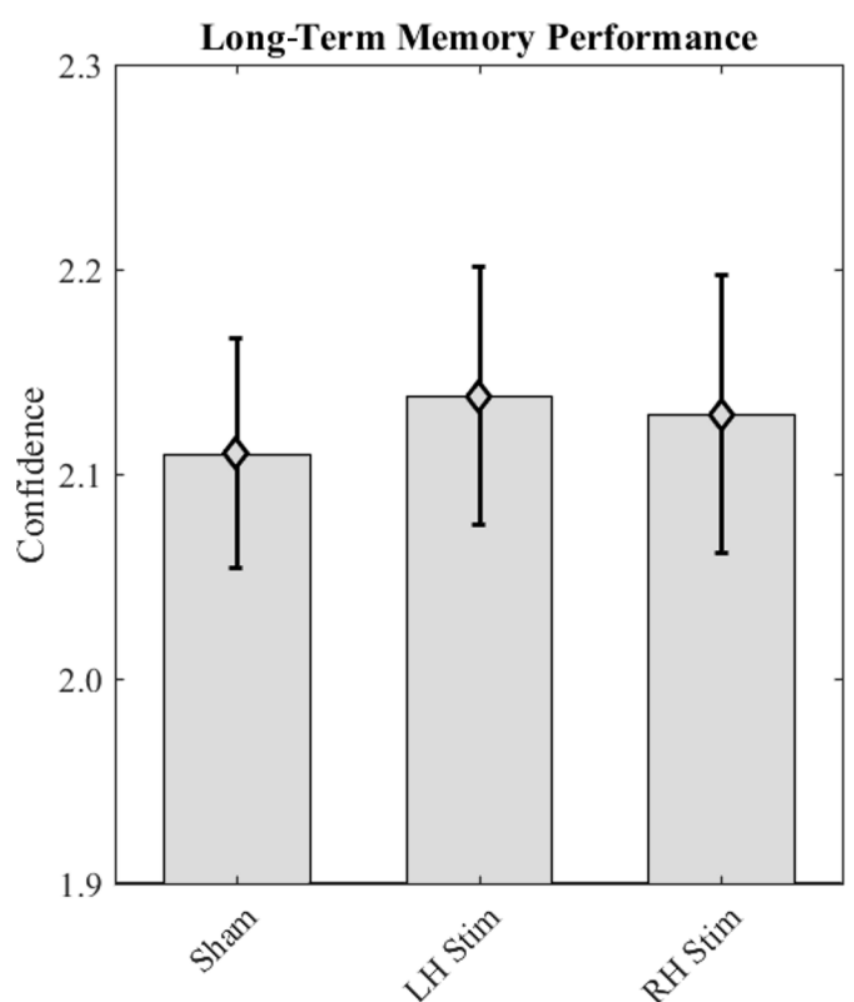

Figure 25: Mean confidence levels during the Long-Term Memory Part. (tACS experiment.) The diagram comprises the data of 25 participants. Each bar plotted on the x-axis illustrates one stimulation condition: sham stimulation (Sham), stimulation of the left hemisphere (LH Stim) and stimulation of the right hemisphere (RH Stim). The y-axis indicates the mean confidence levels that were selected for all decisions during the LongTerm Memory Retrieval. Confidence level 1 represents the option "maybe", 2 stands for the option "probably" and 3 symbolizes "definitely". The error bars display the SEM. (Own illustration.)

As the next step, the performance for the Long-Term Memory Part was determined (see Figure 26). The mean performance for sham stimulation (72 $\pm 1 \%$ SEM) was compared with the mean performance during the left hemisphere stimulation $(73 \pm 2 \%$ SEM) with a nonparametric two-tailed Wilcoxon test and revealed no significant difference $(\mathrm{p} \leq 0.05$; mean performance during LH tACS compared to sham stimulation $\mathrm{p}=0.51$ ). However, for the stimulation of the right TPO area we observed a significant increase in the mean performance $(75 \pm 2 \%$ SEM, Wilcoxon test: mean performance during RH tACS compared to sham stimulation $\mathrm{p}=0.03)$. 


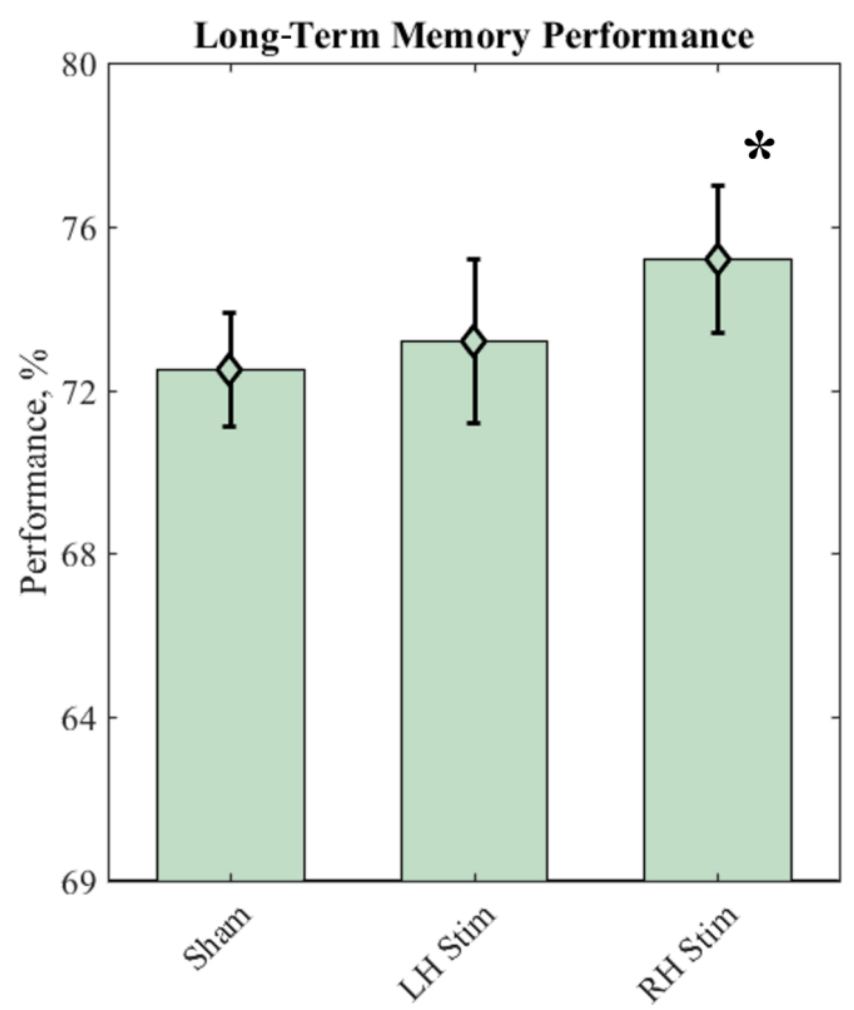

Figure 26: Mean memory performance in the Long-Term Memory Part. (tACS experiment.) The diagram comprises the data of 25 participants. Each bar plotted on the $\mathrm{x}$-axis displays one stimulation condition: sham stimulation (Sham), stimulation of the left hemisphere (LH Stim) and stimulation of the right hemisphere (RH Stim). The y-axis shows the mean performance in percent. The error bars display the SEM. (*) shows statistical significance against sham stimulation (non-parametric, two-tailed, paired Wilcoxon test $\mathrm{p} \leq 0.05$ ). (Own illustration.)

Finally, the weighted performance in the Long-Term Memory Part was calculated by counting correct answers as positive points and wrong answers as negative and multiplying them by the level of confidence (see Figure 27). The points were then averaged, revealing a weighted performance of $209.7 \pm 14.5$ SEM for sham stimulation and 218.4 \pm 19.2 SEM for the stimulation of the left hemisphere. Comparing the weighted performance during tACS of the left TPO area to the weighted performance of sham stimulation, no statistically significant differences were found (non-parametric, two-tailed Wilcoxon test $\mathrm{p}=0.5$ ). During the stimulation of the right hemisphere the weighted performance was $233.2 \pm 18.1$ SEM. There was observed a significant difference between the weighted performance during tACS over the right TPO area and sham stimulation (non-parametric, two-tailed Wilcoxon test $\mathrm{p}=0.04)$. 


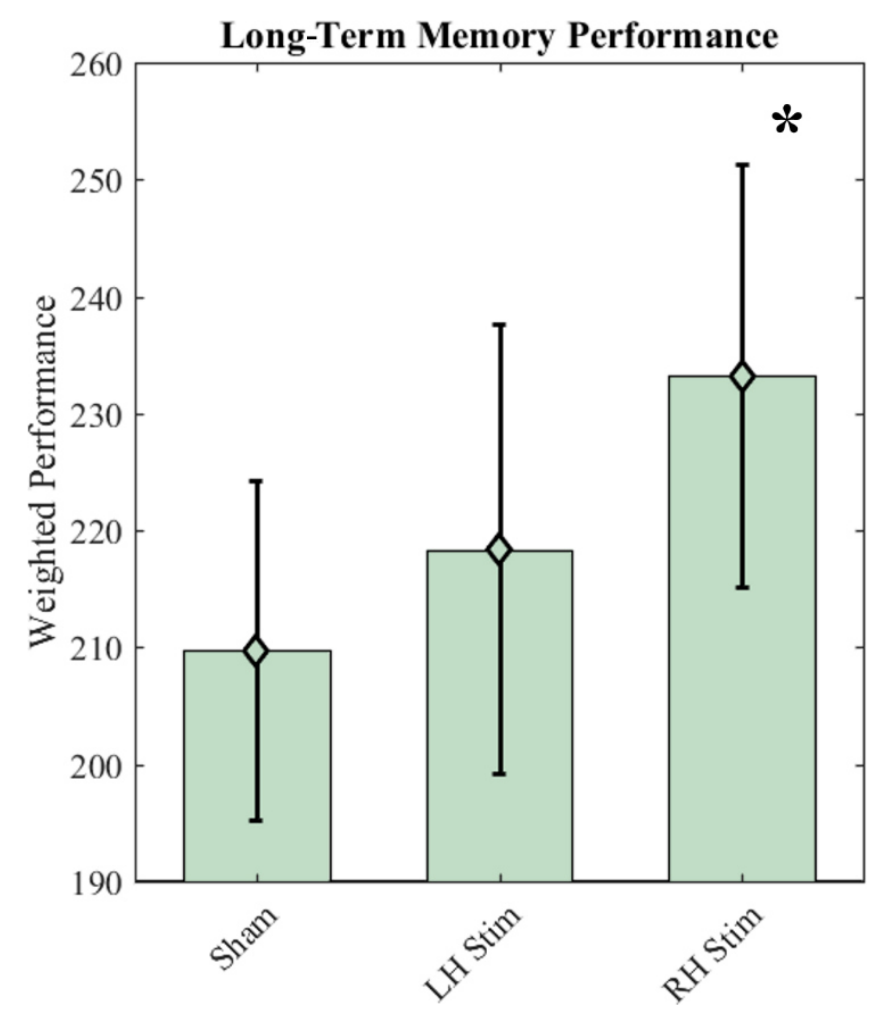

Figure 27: Weighted performance during the Long-Term Memory Recognition. (tACS experiment.) The diagram comprises the data of 25 participants. The weighted performance in the Long-Term Memory Part was calculated by counting correct answers as positive points and wrong answers as negative and multiplying them by the level of confidence. Each bar plotted on the x-axis represents one stimulation condition: sham stimulation (Sham), stimulation of the left hemisphere (LH Stim) and stimulation of the right hemisphere (RH Stim). The y-axis shows the mean weighted performance in points. The error bars represent the SEM. (*) highlights the statistical difference in comparison to the sham stimulation according to the non-parametric, two-tailed, paired Wilcoxon test ( $\mathrm{p} \leq 0.05)$. (Own illustration.)

For an illustration of the effect size of the stimulation of the right hemisphere see Figure 28. The graphic displays the relative change in performance during the stimulation of the right TPO area in comparison with the baseline performance during sham stimulation. It reveals that 18 subjects benefited from tACS over the right TPO area. However, for 5 participants the relative performance decreased, while 2 subjects did not show a response to the stimulation. 


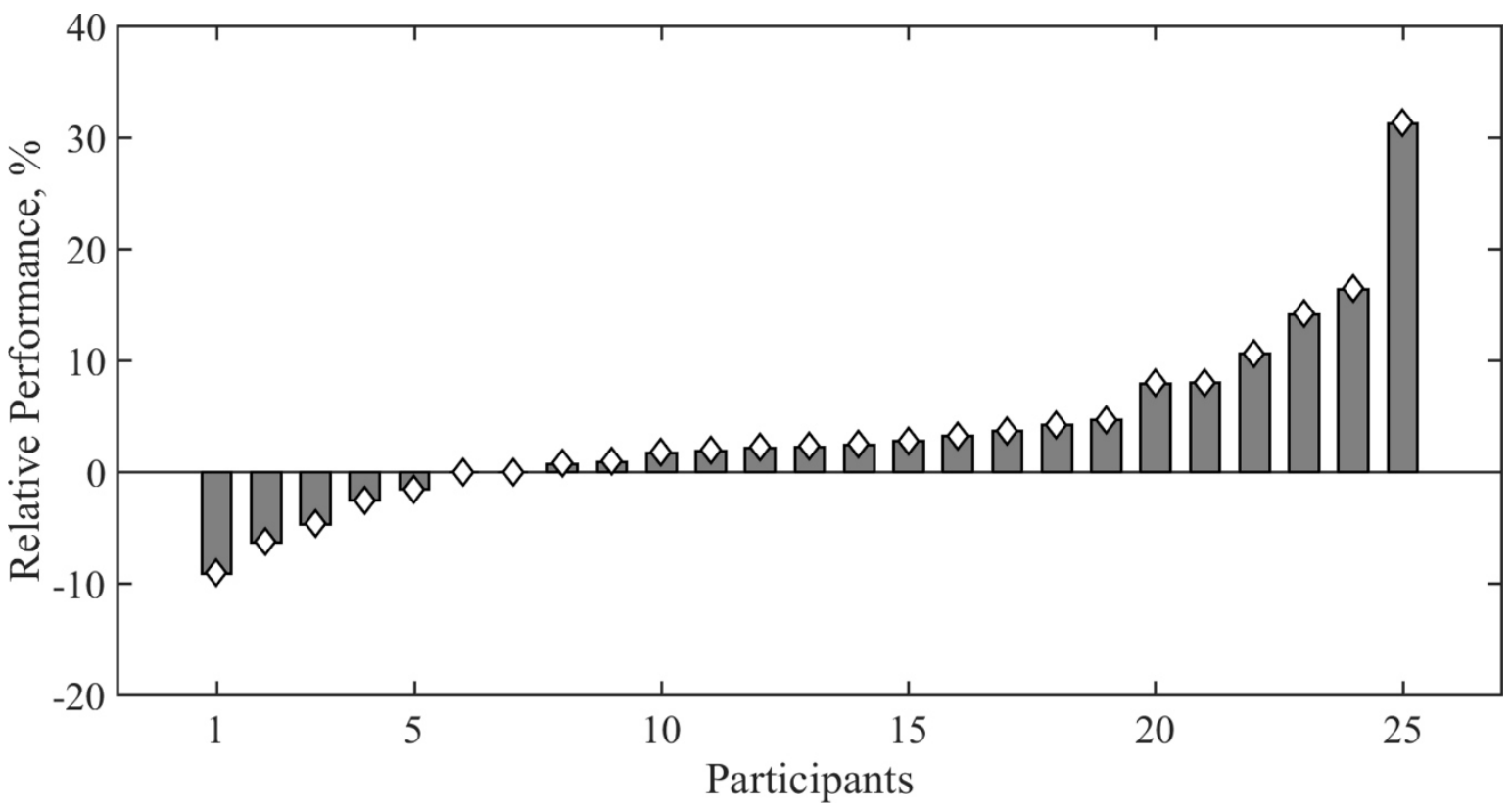

Figure 28: The individual performance (in \%) in the Long-Term Memory Part during tACS over the right TPO area relative to the sham stimulation. The diagram comprises the data of 25 participants. The $\mathrm{x}$-axis lists the number of participants re-arranged according to their performance in an ascending order from worse (left) to best (right). The y-axis indicates the relative performance in percent where the baseline is formed by the performance of every participant during sham stimulation. Negative percentages display a relative decrease in performance, while positive show a relative increase. (Own illustration.)

\subsubsection{Adverse effects}

All participants tolerated the stimulation with tACS well and no session had to be interrupted due to adverse effects. None of the participants reported any phosphenes (the perception of flickering).
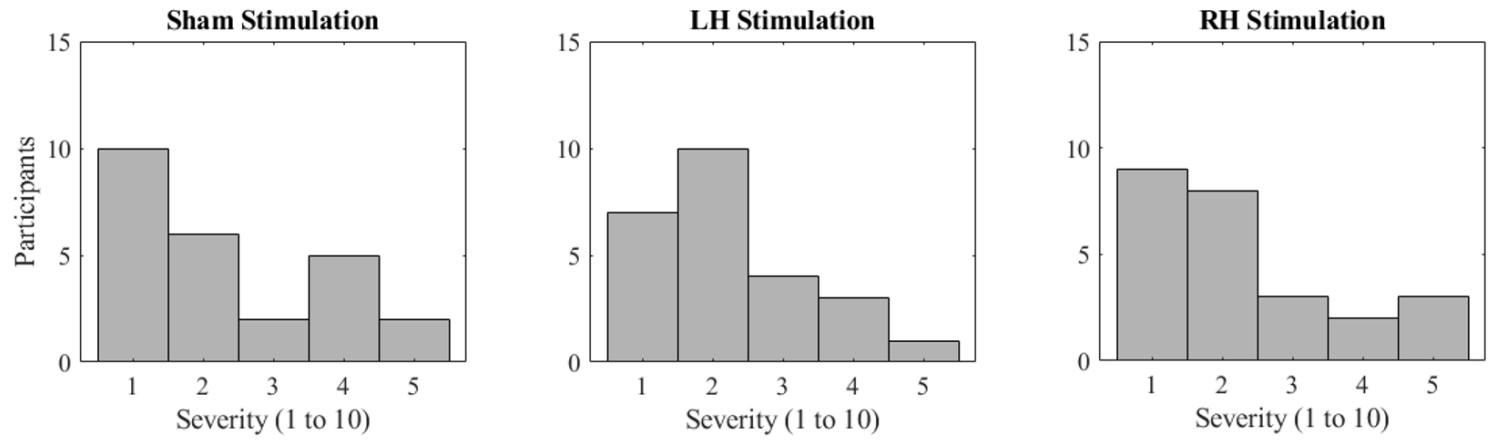

Figure 29: Severity of skin sensations. The $x$-axis depicts the severity of sensations where each bar represents a severity level. Severity ranges from 1 (lowest) to 10 (highest) displayed for all 25 participants in the tACS experiment. The $y$-axis shows the number of participants. The reported sensations are grouped with regard to the stimulation conditions. Left: sham stimulation, Middle: left hemisphere (LH) stimulation, Right: right hemisphere $(\mathrm{RH})$ stimulation. Each bar represents a level of sensation severity. No participant reported a severity higher than 5 points out of 10 . (Own illustration.) 
However, there were reported minor skin sensations. Figure 29 displays the skin sensations for all sessions during the tACS experiment with regard to the severity (from 1 as lowest to 10 as highest). No intense perceptions were reported as none of the subjects declared a severity level higher than 5 . The mean sensation severity level for sham stimulation was $1.56 \pm 1.5$ standard deviation, for the stimulation of the left hemisphere it was about $1.43 \pm$ 1.3 standard deviation and for the right hemisphere there was reported a mean severity of $1.52 \pm 1.5$ standard deviation. A two-tailed, non-parametric, paired Wilcoxon test did not reveal any significant differences for skin sensations between the stimulation of the right nor the left hemisphere in comparison to the sham stimulation (mean sensation severity level during tACS of the right hemisphere in comparison to sham stimulation $\mathrm{p}=0.74$, mean sensation severity level during tACS of the left hemisphere in comparison with sham stimulation $\mathrm{p}=0.7)$. 


\section{Discussion}

Memory consolidation and learning processes are related to plasticity changes and oscillatory activity within and between brain areas. Recording this activity and manipulating it with external sinusoidal currents provide further insights into the relationships between brain oscillations and face encoding in episodic memory. The primary aim of this study was to investigate the oscillatory correlate behind episodic memory encoding processes.

At first, EEG was recorded to determine the activity in a specific brain region as well as an oscillatory frequency that correlates with the successful encoding of faces. In result, we identified the frontal cortex to be significant for the efficient STM encoding of face-moneycombinations in the low theta rhythm $(4 \mathrm{~Hz})$. In contrast, for successful long-term encoding the crucial role of the posterior low theta rhythm in the right TPO area could be revealed. Then, tACS at the frequency of $4 \mathrm{~Hz}$ over the right TPO area and the stimulation of the symmetrical area over the left hemisphere were compared in contrast to the sham stimulation. According to literature, we expected the stimulation, which was applied during the encoding of facial memory items, to entrain internal low theta oscillations and increase the resulting retrieval performance. In line with our expectations, it could be found a significant increase in the performance and the weighted performance in the LTM retrieval of faces ensuing the low theta-stimulation of the right TPO area during encoding, while there could not be observed an effect on performance or reaction time during STM retrieval. The results confirm the initial hypotheses and data analysis let us draw two main conclusions: (i) At first, low theta oscillations in the right temporo-parieto-occipital area during facial encoding reflect the subsequent memory effect. In addition, the right TPO area is unique for facial LTM encoding as successful STM encoding particularly correlates with frontal cortex synchronization. (ii) Secondly, low theta-tACS over the identified right TPO area during encoding increases the LTM recognition performance while it leaves STM retrieval unaffected.

\subsection{The EEG experiment}

During this experimental stage the brain regions and related frequencies that are responsible for the encoding of later successfully retrieved facial stimuli were investigated. Low theta rhythm $(4 \mathrm{~Hz})$ was shown to be positively correlated with successful memory encoding. For the encoding of later correctly remembered face-money combinations into STM our results revealed synchronized low theta activity in the frontal and posterior cortex. The difference underlines the pivotal role of the frontal cortex for the successful encoding in STM. In fact, 
literature has often described the causal role of the frontal cortex with special focus on the PFC in the context of STM. This area is predominantly related to executive functions, attention and mental adaptability (Girotti et al. 2018). The lateral part of the PFC is responsible for the successful completion of cognitive tasks requiring working memory for the active maintenance of items (Barbas 2013). Evidence also comes from studies examining diseased people with impaired PFC and showing correlation with STM deficits (Kumar et al. 2017). For example, in Alzheimer's disease, working memory is impaired and was found with deteriorated plasticity in the dorsolateral prefrontal cortex (DLPFC) (Murray et al. 2017). Our findings broadly support the work of other studies concerning this area, linking prefrontal cortical activity to the performance in STM tasks.

Additionally, the PFC receives input from sensory cortices such as audible, somatosensory and also visual areas (Morris 2013). Besides its importance in cognitive tasks the PFC was proven to be important in social behavior and the processing of emotions. As facial stimuli can clearly be allocated to social and emotional processing networks, our findings are consistent with earlier observations. The synchronous activation of the frontal and the posterior cortex in the encoding of later successfully retrieved answers corresponds to the proposition of several studies that working memory and decision making are highly related to both frontal and posterior parietal areas (Pahor and Jaušovec 2014; Bergmann et al. 2016; Murray et al. 2017). While the posterior parietal cortex is associated with selective processes, the strength of working memory items and the prevention from distraction are dedicated to the PFC (Murray et al. 2017). In sum, our results reveal the pivotal role not only of the PFC exclusively but also of the interaction with posterior cortices for the successful encoding in STM. In contrast to that, synchronized activation in the theta range was found only in the posterior cortex during encoding for later correctly remembered faces in the STM Retrieval Part. This can most likely be explained by an activation of the visual cortex due to the optic processing of facial stimuli in the occipital lobe and gives further strength to the idea that theta activation in the frontal and parietal cortex are decisive for the successful encoding of face-money combinations in STM.

Regarding the question of the successful encoding in episodic LTM, the present study shows that frontal and posterior activation correlate with later correctly retrieved faces. The most interesting finding is that contrasting brain activity for later correctly against later wrongly recognized faces reveals significant synchronization in the low theta spectrum in the left prefrontal cortex and the right TPO area, that is acting as an integration network connecting different cortical areas (De Benedictis et al. 2014). The supposition that the PFC is also causal for the encoding of LTM has already been suggested in earlier work. This importance was 
underlined by a study by Marin et al., who applied prefrontal theta-burst stimulation to impact the retrieval from episodic memory (Marin et al. 2018). Stimulating the DLPFC prior to the performance of a memory task led to a decrease in LTM retrieval, which indicates the organizational role of this area for the episodic memory formation. This was also strengthened by the increased interaction between the PFC and the hippocampus not only during the encoding but also during the retrieval of information (Blumenfeld et al. 2011; Voss et al. 2011). Supposedly in case of LTM encoding, the PFC is responsible for protecting important information from noise and preserving a structural frame that is decisive for the hippocampus to bind information (Marin et al. 2018).

Another key finding of the EEG experiment is the significant activation of the right TPO area during the successful encoding of faces into episodic memory. This can be explained by its fundamental role as an integration area to maintain higher cognitive processes (De Benedictis et al. 2014). A recent tractography study by Wang et al. with high angular diffusion spectrum imaging investigated local connectivity of the TPO area (Wang et al. 2016). They confirmed two tracts that were already described in the literature: the superior longitudinal fasciculus and the ventral occipital fasciculus. Apart from that, they detected a new connection in the TPO area between the fusiform gyrus and the inferior and middle temporal gyrus. This also provides strong evidence for the associative function of the TPO area linking face processing in the fusiform gyrus to brain areas in the temporal gyri that are responsible for LTM processes. All these findings consequently support our conclusion that oscillations in the TPO area indicate the subsequent memory effect during encoding in episodic memory. Another study focusing on brain activity not during encoding but during recognition processes in episodic retrieval was conducted by Kim and Cabeza, in which they recorded fMRI during a word recognition task (Kim and Cabeza 2007). The authors conclude that high-confidence responses are related to activity in the MTL when the former presented word was correctly identified. By contrast, there is observed activity in the frontoparietal cortex when participants were highly confident with false recognized words. The authors suggest that frontoparietal activity is connected to familiarity mechanisms while activity in the MTL reflects LTM processes. Interestingly, this supplements the present results, when there was detected desynchronization in the TPO area during failed encoding and synchronized activity during successful encoding.

The prominent synchronization of the right TPO area in contrast to the "silent" contralateral hemisphere suggests that there is a lateralization for facial stimuli in the right hemisphere, which confirms existing theories in literature (Kanwisher and Yovel 2006; Pitcher et al. 2011; Geib et al. 2017). Other researchers even detected a maximal synchronization of activity in 
the left frontal and the right posterior parietal cortex that agrees with our results (Fell and Axmacher 2011). Additionally, the fusiform face area as part of the temporal lobe was found to be increasingly activated in the right hemisphere in tasks requiring to distinguish between familiar and unfamiliar faces (Weibert and Andrews 2015). Higher activity in this area predicted a successful identification of a familiar face and also corresponds with our results, as we found increased synchronization in the right TPO area during the encoding of subsequently recognized faces. However, this outcome is contradictory to a study by Bergmann et al. who reported a predicting effect of the activation in the left fusiform gyrus for successful encoding (Bergmann et al. 2016). This inconsistency might be due to the type of stimuli used in the study. Bergmann et al. investigated the encoding of object-location relationships. By contrast, our study focused on facial stimuli. Thus, these at first sight conflicting results could be even further evidence that the right TPO area is unique for facial processing. Finally, a conspicuous observation to emerge from the data analysis was the crucial role of low theta band activity $(4 \mathrm{~Hz})$. Oscillations in the theta frequency are widely attributed to cognitive processes, especially to memory formation (Brignani et al. 2013; Antonenko et al. 2016). In accordance with previous studies, we demonstrated that theta synchronization plays a pivotal role for memory encoding (Antonenko et al. 2016). Besides, theta oscillations are also expected to play a fundamental role in synaptic plasticity processes and communication between different brain areas (Brignani et al. 2013). There is broad literature supporting our finding that enhanced theta power during encoding processes is correlated with subsequent retrieval from episodic memory (Osipova et al. 2006; Hanslmayr et al. 2011). Source analysis of a magnetoencephalography study, that investigated thetasynchronization in case of associative face-scene pairs, found a theta activity increase in prefrontal, temporal and occipital cortices (Düzel et al. 2010). This confirms our findings of synchronized theta activity in the left PFC and the right TPO area and strengthens the assumption of their importance for episodic memory encoding in general and facial encoding in particular. Furthermore, investigation in neurosurgical patients showed that enhanced theta phase coupling between cortical and hippocampal areas during encoding and retrieval increase successful memory retrieval (Pastötter and Bäuml 2014).

In summary, the results of this stage share a number of key features with literature and complement earlier work on the field. However, as most of the studies in the field, this experiment provides only correlational evidence for the impact of low theta oscillations in the right TPO area on facial memory encoding. To provide decisive causal proof, we used tACS to stimulate the right TPO area and surveyed behavioral consequences for facial LTM encoding. 


\subsection{The tACS experiment}

Based on the results of the prior experimental stage, we designed an experiment using tACS to manipulate the encoding of faces in episodic LTM. Due to the increased synchronized activity in the right TPO area and the left PFC, there were two options for potential target regions. As we aimed to stimulate a region specific for LTM, the PFC was unsuitable because EEG revealed it to be not exclusively activated during LTM encoding but also during STM processes. Hence, the right TPO area, which specifically showed synchronized low theta activity for LTM encoding, was stimulated with low theta-tACS. For active control, the left TPO area was stimulated with the same parameters. Finally, the TPO areas of both hemispheres received sham stimulation to control for possible placebo effects.

For STM, as expected, we observed no changes, neither in accuracy nor in reaction time. This finding reconfirmed our hypothesis that the correlation of synchronized low theta activity within the right TPO area is unique for LTM. However, there are contradictory studies, suggesting that posterior cortical regions also play a role for reaction times in STM tasks. For example, Hopfinger et al. stimulated the right inferior parietal cortex with 10 and $40 \mathrm{~Hz}$ to investigate alpha and gamma oscillatory activity in attentional processes in a visual attention task and found a significant improvement in conscious reaction time for the stimulation with $40 \mathrm{~Hz}$ (Hopfinger et al. 2017). However, the frequency that they detected to have a significant effect belongs to the gamma frequency range, while we investigated the low theta range. Another study by Pahor and Jaušovec agrees with our results on LTM, as authors found an effect of theta-tACS on the left frontal and parietal cortex in fluid intelligence as a parameter for STM (Pahor and Jaušovec 2014). They attributed increased attention to the frontal theta-tACS, while storage enhancement was attributed to parietal theta-tACS. Our study extends their findings to the concept of LTM and moreover corroborates effective entrainment of internal low theta oscillations.

The most important finding in this experimental stage is the significant improvement in memory performance for the transcranial electric stimulation of the right TPO area during encoding compared with the left TPO area or sham stimulation. The results provide evidence for an explicit causal relation between low theta oscillatory activity in the right TPO area and episodic memory encoding. The findings confirm our second hypothesis that online tACS changes neural oscillations in the TPO area, which has a direct effect on behavior. Increased performance in memory tasks due to low theta-tACS supports evidence from other studies when tACS in the theta range was used to investigate cognitive processes indicating a positive correlation between theta power and memory performance (Brignani et al. 2013; Lisman and 
Jensen 2013; Pahor and Jaušovec 2014; Clouter et al. 2017). This is the first study not only describing a correlation between low theta oscillations in the TPO area and the successful episodic memory encoding of faces but also presenting evidence for causality by manipulating with low theta frequency. Our results provide significant contribution to the literature, linking theta oscillations in the temporoparietal cortex to memory encoding processes (Antonenko et al. 2016). For instance, Antonenko et al. conducted a shamcontrolled pilot study using theta-tACS at the frequency of $6 \mathrm{~Hz}$ during an implicit learning task with associations between objects and pseudowords to simulate language learning. In contrast to our study, stimulation was applied to the temporoparietal cortex of the left hemisphere and induced a significant increase in memory performance. Their results agree with our hypothesis, even though they detected an improvement for the opposite hemisphere. It seems highly likely that this lateralization can be attributed to the difference in stimuli category, as the left hemisphere is assumed to be responsible for verbal stimuli and language learning, while the right hemisphere processes pictorial stimuli and faces (Pitcher et al. 2011; Geib et al. 2017).

Contrary to expectations, no significant differences were found in the mean confidence level between stimulation and sham. This is somewhat surprising considering that accuracy was significantly improved in LTM recognition. We can speculate that the increase in accuracy was part of processes on an unconscious level and cannot be translated to the conscious perception of the confidence. Interestingly, statistical analysis detected a significant increase in weighted performance for the stimulation of the right hemisphere in contrast to the left hemisphere. This proves that the rate of very confident and correctly classified answers was significantly higher for the stimulation of the right TPO area revealing that at least confidence levels for correct answers significantly increased.

A recent study by Romanska et al. also investigated the stimulation of a similar brain target, the lateral occipitotemporal cortex, to affect facial identity perception and trustworthiness perception (Romanska et al. 2015). They applied 20 minutes of high-frequency tRNS or sham stimulation and found an enhancement in the encoding of unfamiliar faces, but not in trustworthiness. In the second experiment, spatial specificity was proven by stimulating the occipitotemporal in contrast to the sensorimotor cortex. Even though they used another non-invasive brain stimulation method, their results agree with our findings. On the one hand, they confirm our finding that the right posterior area is involved in the LTM encoding of facial stimuli. On the other hand, Romanska et al. also observed enhanced recognition, showing that $\mathrm{tES}$ techniques are a valuable tool to manipulate with memory formation. 
Our results contribute to the mechanistic understanding of memory processes, underlying oscillatory activity, and behavior. As theta oscillations are attributed to memory and synaptic plasticity, it can be drawn certain conclusions about the effect of tACS on operational encoding processes. Reinforcing low theta oscillations facilitates the encoding of faces in episodic memory, which agrees with multiple studies that have shown memory to be dependent from oscillatory theta power (Osipova et al. 2006; Guderian et al. 2009; Vosskuhl et al. 2015). In fact, tACS can influence the memory encoding by entraining related brain oscillations in a frequency- and location-specific manner (Polanía et al. 2012). Internal oscillatory activity gets synchronized with externally applied alternating current (Herrmann et al. 2013). Thus, subthreshold signals from neuronal cells might be potentiated by tACS in the applied frequency range to exceed the threshold. With the application of low theta-tACS, faces that failed to be successfully encoded due to insufficient signal-to-noise strength can be rescued by tACS, which entrains internal oscillations and increases the signal amplitude to reach suprathreshold activity. Furthermore, tACS-induced synchronization within and also between brain networks might lead to expedited information transfer (Schutter 2014). As we stimulated the TPO area, which has rich connections to other regions, the successful encoding of faces potentially arises due to strengthened inter-regional communication. Moreover, theta-tACS is suggested to increase cortical communication with more distant brain areas being involved in episodic encoding (Fell and Axmacher 2011; Kasten et al. 2016). The brain imaging study by Wang et al. revealed a new junction between the TPO area and the MTL, where the hippocampus is located (Wang et al. 2016). Thus, it can be assumed that externally applied theta-tACS intensifies information transfer and exchange between the TPO area responsible for face processing and the hippocampus that encodes information into LTM. Besides, theta synchronization between frontal and posterior brain areas is known to play a role in LTM encoding, which is also in line with our EEG study, that revealed the left PFC and the right TPO area to be related with successful encoding. Accordingly, thetatACS most probably reinforced these communication processes, leading to a higher memory performance. The present results are also in line with the causal role of theta oscillations in the MTL, where enhanced prestimulus theta amplitudes during encoding predict improved memory recall (Guderian et al. 2009). In accordance with our findings, Guderian et al. demonstrated the importance of theta oscillations for episodic memory performance but not for the reaction time. Guderian et al. hypothesized that reaction times are an indicator for the arousal level rather than memory encoding. This agrees with our interpretation that stimulating the right TPO area revealed significant changes in memory processes resulting in 
an increased LTM performance irrespectively of the level of arousal that the facial image induces and most probably due to subconscious alterations in memory formation.

Altogether, our results are consistent with data obtained in former studies, enabling tACS to manipulate with cognitive processes, impact memory encoding, and increase the subsequent memory effect. Our study further expands knowledge on low theta rhythm in the right TPO area by showing increased accuracy and weighted performance in a facial memory recognition task due to low theta-tACS.

\subsubsection{Control for side and adverse effects}

We used standardized questionnaires after every session to assess potential side and adverse effects (Antal et al. 2017). None of the participants reported any intense side effects during the stimulation. The mean level of skin sensations (itching, tingling, burning) was below two on a 10-point scale where 10 is the highest. Any individual indicator was not stronger than five. These relatively weak perceptual effects are in line with other studies, where no serious adverse effects were reported (Schutter 2014; Fertonani et al. 2015; Antal et al. 2017). Moreover, perceptional impressions declared in the questionnaires were not different between all three conditions: tACS over the right hemisphere, tACS over the left hemisphere, or sham stimulation (Kruskal-Wallis test $\mathrm{p}=0.94$ ). No flickering sensations were reported by the volunteers and no adverse effects were registered. In summary, our study confirms tACS as a non-invasive and painless method to focally and reversibly entrain cortical oscillations.

\subsection{Clinical relevance}

With a view to the health care system, tACS application gains greater significance for therapeutic purposes as it comes along with low costs, a comparatively easy handling, and little side effects (Antal and Paulus 2013; Brignani et al. 2013; Brunoni et al. 2013; Schutter 2014). In this context, our results are of specific importance regarding psychiatric and neurological disorders, where face recognition and LTM are particularly affected. For example, neurocognitive disorders such as autism and schizophrenia were described to show an impairment in holistic face processing (Weigelt et al. 2012; Watson 2013). These patients are suffering from a disabled face memory and perform worse at individuating faces. Special emphasis at that point should be placed on prosopagnosia, often referred to as face blindness, that is characterized by a cognitive deficiency in face processing abilities, while intellectual and perceptual skills are on a normal level. Patients suffer from a visual agnosia selectively 
for faces (Corrow et al. 2016). They are not able to recognize faces - even very familiar faces, from their childhood, or their own. However, prosopagnosics can identify a face as such and derive information like emotional status and gender from it (Kennerknecht et al. 2006). A distinction is made between a congenital form present from birth, most probably related to so far unknown genetic alterations, and an acquired form due to brain damages (Corrow et al. 2016). It is suggested that congenital prosopagnosia follows an autosomal dominant inheritance, so that affected persons provide at least one first degree relative also suffering from face blindness (Kennerknecht et al. 2006; Grüter et al. 2008). In acquired prosopagnosia structural lesions are caused by degenerative atrophy, cancer, encephalitis, trauma, or stroke (Corrow et al. 2016). They mainly affect the occipital-temporal lobe that is highly involved in facial processing (Gainotti and Marra 2011; Corrow et al. 2016). Moreover, lesions at the temporal-parietal junction were identified to cause prosopagnosia (DeGutis et al. 2014). In accordance with these findings, our study has revealed a causal connection of the temporal, parietal, and occipital lobe with the encoding of faces in episodic memory. Moreover, our results indicate the crucial role of low theta synchronization particularly in the right TPO area. This matches to earlier observations of patients with acquired prosopagnosia revealing lesions in the right posterior cortex, while damages of the corresponding area in the left hemisphere did not lead to prosopagnosia (Grüter et al. 2008; Gainotti und Marra 2011). As face recognition abilities are prerequisites for social behavior in human society, prosopagnosia is related to drastic limitations for interaction and daily functioning in private and professional life. At worst, possible consequences are chronic anxiety and the feeling of social isolation and depression (Corrow et al. 2016). A study by Kennerknecht et al. revealed a prevalence of prosopagnosia of $2.5 \%$ in the Caucasian population (Kennerknecht et al. 2006). This emphasizes the importance of novel therapeutic options. So far, the most therapeutic interventions focus on the training of coping strategies (DeGutis et al. 2014; Corrow et al. 2016). For example, patients with congenital prosopagnosia have developed compensatory strategies to identify their counterpart by cues such as haircut, clothes, voice, or gait (Kennerknecht et al. 2006). However, this treatment provides inconsistent outcomes. As the results confirmed the causal relationship between low theta-tACS over the right TPO area and the successful face encoding in healthy young adults, it can be argued that the application of low theta-tACS is a viable strategy for the therapy in patients with prosopagnosia. A comparable approach was pursued by Wilkinson et al. using galvanic stimulation on the vestibular system resulting in a significant improvement in performance of a prosopagnosic patient in a forced-choice face-matching task (Wilkinson et al. 2008). Probably, improvement in facial encoding might also be applicable to patients suffering from 
impaired face processing in autism spectrum disorder, Turner's syndrome, schizophrenia, and Parkinson's disease (DeGutis et al. 2014; Corrow et al. 2016). Other application aspects could be Alzheimer's disease and cognitive decline in geriatric patients. Considering the wide range of application, our findings have important implications for the development of innovative therapeutic strategies for the treatment of face blindness and memory deficit.

\subsection{Strengths of the study}

In comparison to other NIBS methods, tACS provides plenty of advantages. It allows us to entrain and manipulate internal oscillations in a frequency-specific manner (Antal and Paulus 2013). Regarding other techniques, tACS has a greater specificity, compared with tDCS, and less adverse effects, compared with TMS (Rossi et al. 2009; Ali et al. 2013). Accordingly, sensations due to the sinusoidal current are very subtle (Tavakoli and Yun 2017). This also assures effective blinding and makes it impossible for the study participants to differ between placebo or sham stimulation and real stimulation. A low rate of side effects was also confirmed in our study, which makes the chance of a placebo effect being responsible for significant differences in behavior very unlikely. A key strength of our tACS study is the double-blinded, placebo-controlled, randomized, counterbalanced, and crossover design. The high effectiveness of 72 percent (18 subjects out of 25 significantly benefitted from the stimulation) underlines the weight of the study. Another strength of the study is the conduction of the EEG and tACS experiments in independent groups to reach the best possible generalizability of the principal findings. To reduce uncertainty or variability in the results, there were taken several precautions. First, to guarantee a stable environment, all sessions were conducted in the same laboratory with the same setting regarding devices, participant's position, and lighting. The volunteers were familiarized with the environment and task before the first experimental session. Second, the enrollment routine included exclusion criteria, comprehensive questionnaires (see Appendix), and a basic medical examination; thus, all participants had a comparable health status (healthy young adults). To verify the exact and equal positioning of the stimulatory electrodes, we used special electrode caps of individualized size. As previous research has revealed that operating with smaller electrodes instead of sponge electrodes with a surface of $35 \mathrm{~cm}^{2}$ increases current focality; accordingly, we used multiple electrodes with a radius of $1 \mathrm{~cm}$ (Herrmann et al. 2013). Additionally, smaller electrodes deliver current with less sensations, which also contributes to minimal side effects and a successful blinding (Fertonani et al. 2015). 
Although there has been previous research on the topic of episodic memory, most studies focused on a correlative link between neuronal oscillations and cognitive processes. With the present study, we go one step further by not only providing correlative evidence for the critical role of temporo-parieto-occipital low theta oscillations in episodic encoding processes but also by adding a causal proof. We revealed an increase in LTM retrieval performance due to manipulation in the mentioned frequency spectrum. Delivering the same strength of the current to both hemispheres only showed a significant effect for the TPO area in the right hemisphere and is in accordance with EEG data from the prior experimental stage. At last, our results prove the specificity of the effect for LTM by contrasting it to STM retrieval performance and reaction times, that did not show significant changes under tACS in comparison to sham.

\subsection{Limitations of the study}

A few limitations should be considered. First of all, a limitation of every EEG study is that even if postsynaptic potentials form the largest proportion of the measured signal, there are existing other sources in the brain having an influence on the generation of LFPs (Cohen 2017). Accordingly, glial cell activity and spiking activity of sodium and calcium channels contribute to the neural signal as well, which complicates the interpretation of the findings. Moreover, its high temporal resolution, being a major benefit for the experimental design in our study, accompanies with a relatively poor spatial resolution (Lopes da Silva 2013). Thus, it is hard to attribute observed low theta activity to one specific sub-region (e.g., gyri). However, these general constraints of EEG have a limited effect on our study. We used a 128-channel active EEG system, providing the maximum possible spatial resolution and data quality according to the current state of the art. Besides that, the present study has only examined the behavioral effect of tACS. We did not carry out EEG measurement during the stimulation because a parallel application is still under-developed, and the distinction between brain activity and stimulatory artefacts due to the electric field induced by the current stimulation is problematic (Noury and Siegel 2018; Gebodh et al. 2019). However, such recordings would be possible, they would have served as a direct proof for the entrainment of oscillations, as behavioral changes only provide an indirect account for it.

In future studies, the experimental protocol can be enhanced using electrocorticography, where subdurally implanted electrodes record brain activity. In contrast to non-invasive methods, this technique provides maximal temporal and spatial resolution, is less distorted by artefacts, and has a higher signal-to-noise ratio (Hill et al. 2012). For obvious ethical 
reasons this method is not appropriate in research with healthy humans and stays preferred method in animal research and in research with epileptic patients. Nevertheless, it was proven that the application of weak electric current on the skull of mice and monkeys affects neuronal spiking and subthreshold activity of underlying brain networks (Ozen et al. 2010; Vöröslakos et al. 2018; Krause et al. 2019). Another point to consider is that we applied stimulation with fixed parameters without adapting the protocol to interindividual variability. Every participant received tACS with the same montage and at the frequency of $4 \mathrm{~Hz}$. Several studies noticed that there are interindividual differences in the internal brain frequency spectrum (Herrmann et al. 2013). Nevertheless, our study aimed to identify a determining frequency of universal applicability to the general population. Interindividual adaptations would limit the generalizability of the results and may not advance the development of broadly applicable clinical use. However, further projects regarding the individualization of tACS could improve the effect size and success rate of our results.

Other factors with impact on the stimulatory effects of tES are genetic and anatomical variability with in consequence individual transmitter concentration and equilibrium as well as variable skull and scalp thickness, cerebrospinal fluid volume, and gyral folding affecting the distance between electrode and cortex and determining the electric conductivity (Brignani et al. 2013; Schutter 2014; Santarnecchi et al. 2015).

Moreover, there should be considered possible intersexual differences in facial processing. Previous studies could identify a higher level of arousal, a larger electrocortical responsivity to human faces and increased activation of the FFA in women in comparison to male subjects (Proverbio et al. 2008; Proverbio 2017). It is also worth noting that the opposite sex preference, that was repeatedly detected in facial experiments with heterosexual individuals, could impact the encoding of faces (Kranz and Ishai 2006). We can speculate that for heterosexuals there might result a higher recognition for faces with the opposite sex. However, in this study neither we asked for sexual preferences nor we performed calculations for sex-dependent accuracy in memory retrieval. Nevertheless, the election of facial images for our study was subject to criteria such as precluding very conspicuous faces, accessories, visible make-up as well as faces with emotional expressions to optimally control for affective or sexual stimuli. As former studies also detected a higher arousal in women for faces of children and elderly, corresponding images were excluded from items for the memory task as well (Baron-Cohen and Wheelwright 2004; Proverbio 2017). The participants who did not show an improvement after brain stimulation in our study were of different self-reported genders. However, we did not carry out an analysis of the data with regard to gender-specific differences. 
Consequently, the inconsistency of the effect size of our tACS study could be attributed to these mentioned aspects. While 18 participants benefited from the stimulation, for 5 people, performance relatively decreased, and 2 subjects showed no response to the stimulation. With establishing a stable laboratory environment, a universal experimental protocol, and several conditions of participation such as interdiction of the consumption of caffeinated drinks and alcohol and enough sleep the night before a session, we provided sensible control over inter- and intraindividual factors.

Although the study has successfully demonstrated an effect on facial LTM, it has certain limitations in terms of specificity for an exclusive effect of the stimulation only on facial encoding in contrast to other stimuli and tasks reflecting LTM processes. Conclusively, we cannot unambiguously demonstrate the specificity of the stimulatory effect on facial learning in episodic memory encoding. Further studies might detect a positive effect on broader spheres of episodic memory. Considering the specificity of the stimulatory effect for the elected frequency and the target brain area, our experimental parameters (low thetastimulation on the right TPO area) were generated based on the results of the EEG study for episodic LTM encoding. While the analysis of the EEG experiment comprises the whole frequency range, significance was only reached for theta oscillations at $4 \mathrm{~Hz}$. The unique role of low theta frequencies was then also reinforced by the tACS experiment. Considering both experiments, the results suggest the causal role of low theta oscillations for memory encoding; nevertheless, further studies testing other stimulatory frequencies apart from $4 \mathrm{~Hz}$ are needed to prove either its uniqueness in the process or the potential involvement of other frequencies.

Regarding the generalizability of the study, it remains to be further investigated whether results in healthy young people can be transferred to other population groups, such as elderly and clinical patients. On the one hand, age and health status have a considerable impact on brain structure, neuronal dynamics, and synaptic plasticity and could therefore lead to different effects of the same stimulation parameters (Antonenko et al. 2016). On the other hand, normalizing and improving brain activity seems more achievable and beneficial in patients with disorders rather than in healthy volunteers.

\subsection{Conclusion and outlook}

The purpose of the current study was (i) to determine a specific brain area that is related to the successful encoding of faces in episodic memory and (ii) to establish the causality between the identified activity and episodic LTM by using multi-electrode tACS. Previous research 
has shown a correlation between theta oscillations and episodic memory formation, but our study extends these finding by taking an interventional approach. At first, we used highdensity EEG during the memory task to pinpoint the crucial activity regarding the successful LTM. We identified a specific brain area (right TPO area) and a frequency $(4 \mathrm{~Hz})$ for facial encoding and investigated the effect of tACS on this encoding process, confirming our first hypothesis that low theta oscillations reflect the subsequent memory effect. In the next experiment, we applied $4 \mathrm{~Hz}$-tACS on the right and the left TPO area in contrast to sham during the encoding part of the task to test the causal behavioral effect. In result, we can validate our second hypothesis that low theta-tACS over the right TPO cortex significantly and reliably improved the encoding in episodic LTM, as recognition performance and performance-weighted confidence significantly increased. The importance of the study is not only derived by the fact that it proved causal evidence for low theta oscillations in facial LTM encoding but also by revealing that entraining low theta by externally applied weak currents can enhance brain processing. This holds promise for a therapeutic application in patients with impaired face memory and recognition. Overall, the study strengthens the idea that tACS is a safe and highly suitable tool for non-invasively exploring higher cognitive processes, demonstrating causality between behavior and oscillatory activity in specific brain regions as well as for boosting plastic changes in memory formation. It offers new insights in the role of theta oscillations in memory processes and raises the question if repetitive stimulation can increase the effect size and induce long-lasting plastic effects. More broadly, exact mechanisms behind tACS still remain an area of open research and a procedural gold standard is not yet ubiquitous. The promising results of our study should be further explored in future experiments and expanded regarding stimulation parameters and group sampling. Moreover, further studies should scrutinize the mechanisms of action to make progress in the establishment of tACS as an auspicious non-invasive and reversible stimulation technique. Aside from scientific basic research, our results have considerable weight for the further development of clinical applications of tACS for therapeutic purposes in cognitive diseases in general and in particular for patients suffering from face blindness such as in prosopagnosia and prosopamnesia. Future work will determine optimal stimulation parameters to induce maximal cognitive performance and investigate how tACS can be combined optimally with other therapeutic strategies, whether pharmacological or face training approaches, to establish the best possible treatment for patients. 


\section{Summary}

In recent years, the investigation of memory formation and retrieval has attracted increasing interest. As oscillatory activity plays a crucial role in neuroplastic processes, episodic memory is to a considerable extent attributable to synaptic changes, synchronization, and neurophysiological alterations through oscillating electric fields. Perception processes are part of episodic memory encoding. Human face perception and encoding arouse particular interest due to their fundamental relevance in social behavior. This study aimed to determine the causal role of brain dynamics in the encoding of facial episodic memory in humans. As recent studies revealed an enhancement in cognitive processes by the entrainment of internal brain oscillations, tACS stepped up as a new method of non-invasive brain stimulation to induce neuroplasticity (Antal et al. 2008; Antal and Herrmann 2016). It is a promising tool to test the role of brain oscillations on episodic memory encoding in humans and the potential for memory improvement.

For the entire study, we developed a memory task that includes encoding, a Short-Term Memory Retrieval Part, maintenance, and a Long-Term Memory Retrieval Part. In the longterm face recognition, we assessed both the performance and the choice confidence on the 3-point scale. Two consecutive experiments were performed. For the first experiment (20 participants), we used 128-channel EEG to identify the region of the brain that is exclusively responsible for the long-term face encoding and the frequency of the brain rhythm that is associated with the successful subsequent recognition. Then, we conducted the tACS experiment (25 participants) based on the frequency and spatial data from the EEG experiment. We implemented a double-blinded, randomized, counterbalanced, crossover, and placebo-controlled study design. 20 minutes of $4 \mathrm{~Hz}$-tACS at $3 \mathrm{~mA}$ peak-to-peak were applied during the encoding task to the identified right or to the left TPO area for active control. One more session included sham stimulation to one or the other area.

The EEG study revealed a significant synchronization of brain oscillations during successful long-term facial memory encoding in the right TPO area at the low theta range (4 Hz). In complete agreement, the placebo-controlled tACS study showed a significant enhancement of long-term memory recognition performance after the low theta-stimulation of the right but not the left TPO area.

The results indicate that low theta oscillations in the right TPO area are vital for successful episodic long-term memory encoding of facial stimuli. Secondly, we confirm that active low theta-tACS applied on this area during encoding improves the subsequent memory recognition performance. This supports the concept of lateralization for face processing in 
the right posterior brain region; moreover it puts forward this area as a crucial neocortical node in communication with the hippocampus for the long-term memory encoding (Pitcher et al. 2011; Geib et al. 2017). The results are in agreement with other studies that revealed a positive correlation between theta power and memory performance (Pahor and Jaušovec 2014; Clouter et al. 2017). However, the present work reveals a causal link between the empowered low theta in the right TPO area and enhanced subsequent long-term memory recognition. In summary, tACS is a highly suitable non-invasive tool to entrain local neocortical low theta activity and enhance long-term memory encoding, which is important in the clinical context for revealing novel therapeutic strategies in prosopagnosia and prosopamnesia. 


\section{Appendix}

Edinburgh Handedness Inventory (Oldfield 1971)

PID:

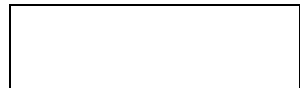

DATE:

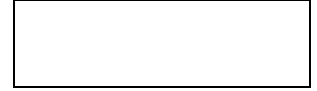

Zeigen Sie bitte welche Ihrer Hände Sie bei den folgenden Tätigkeiten bevorzugen, indem Sie in der entsprechenden Spalte ein + eintragen. Wo die Bevorzugung einer Hand so stark ist, dass Sie nie versuchen würden, die andere Hand dafür zu verwenden, es sei denn, Sie würden dazu gezwungen, tragen Sie ein ++ ein. Für den Fall, dass Sie wirklich keine Ihrer Hände bevorzugen, tragen Sie + in beide Spalten ein. Manche Aktivitäten benötigen beide Hände. In diesen Fällen steht der Teil der Aufgabe oder das Objekt, für den bzw. das Sie entscheiden müssen, welche Hand Sie bevorzugen, in Klammern.

Bitte versuchen Sie alle Fragen zu beantworten und tragen Sie nur nichts ein, wenn Sie keinerlei Erfahrung mit dem Objekt oder der Aufgabe haben.

\begin{tabular}{|l|l|l|}
\hline & Links & Rechts \\
\hline schreiben & & \\
\hline zeichnen & & \\
\hline werfen & & \\
\hline Schere & & \\
\hline Zahnbürste & & \\
\hline Messer z.B. beim Brotschneiden & & \\
\hline Löffel & & \\
\hline Besen (obere Hand) & & \\
\hline Streichholz anzünde (Streichholz) & & \\
\hline Schachtel öffnen (Deckel) & & \\
\hline
\end{tabular}


Auswertung (bitte frei lassen)

\section{Summe}

\begin{tabular}{|l|l|}
\hline & \\
\hline
\end{tabular}

Laterality Quotient L.Q.$=\left(\sum \mathrm{R}-\sum \mathrm{L}\right) /\left(\sum \mathrm{r}+\sum \mathrm{L}\right) \times 100$

Figure A.1: Edinburgh Handedness Inventory. The questionnaire serves as a measuring instrument to determine the laterality of a person's favored hand in everyday activities. 
Zentrum Neurologische Medizin, Klinik für Klinische

Neurophysiologie

Robert-Koch-Str. 40, 37075 Göttingen

UMG-NEUROPHYS

Direktor: Prof. Dr. med. Walter Paulus

Tel.: 0551/3966650

Fragebogen zur transkraniellen Gleichstrom / Wechselstrom / Magnetstimulation

Lesen Sie sich zu Ihrer eigenen Sicherheit diesen Fragebogen gründlich durch und beantworten Sie gewissenhaft alle Fragen. Wenn Sie sich nicht sicher sind oder eine Frage nicht verstehen, wenden Sie sich bitte an einen unserer Mitarbeiter. Unterschreiben Sie anschließend den Fragebogen und lassen Sie sich von einem Mitarbeiter einweisen, bevor Sie an der Studie teilnehmen können.

Anweisung. Bitte vollständig und gut lesbar ausfüllen. Bitte kreuzen Sie immer die Antwort an.

\begin{tabular}{|c|c|c|}
\hline 1 & Sind Sie jünger als 18 ? & $\square$ JA $\square$ NEIN \\
\hline 2 & Ist bei Ihnen ein Anfallsleiden (Epilepsie, inkl. kindlicher Absencen) bekannt? & $\square$ JA $\square$ NEIN \\
\hline 3 & $\begin{array}{l}\text { Ist in Ihrer unmittelbaren Familie (Eltern, Geschwister) eine Epilepsie } \\
\text { bekannt? }\end{array}$ & $\square$ JA $\square$ NEIN \\
\hline 4 & Haben Sie schon Ihr Bewusstsein verloren? Wenn ja, wann? & $\square$ JA $\square$ NEIN \\
\hline 5 & $\begin{array}{l}\text { Sind bei Ihnen andere neurologische oder psychiatrische Erkrankungen (inklusive - } \\
\text { Alkohol-, Medikamenten- und Drogenabhängigkeit oder -mißbrauch) } \\
\text { bekannt? Wenn ja, welche? }\end{array}$ & $\square$ JA $\square$ NEIN \\
\hline 6 & $\begin{array}{l}\text { Leiden Sie unter einer schweren Erkrankung der Atemwege, des Herz- } \\
\text { Kreislaufsystems oder des Bewegungssystems (z.B. Asthma, Diabetes, } \\
\text { Herzschwäche, Herzrhythmusstörungen, Lähmungen)? Wenn ja, welche? }\end{array}$ & $\square$ JA $\square$ NEIN \\
\hline 7 & $\begin{array}{l}\text { Wurde bei Ihnen je zu diagnostischen Zwecken ein EEG oder MRT } \\
\text { gemacht? }\end{array}$ & $\square$ JA $\square$ NEIN \\
\hline 8 & Hatten Sie je behandlungsbedürftige Kopfverletzungen? & $\square$ JA $\square$ NEIN \\
\hline 9 & Leiden Sie regelmäßig an Kopfschmerzen? & $\square$ JA $\square$ NEIN \\
\hline 10 & Haben Sie in der Vergangenheit an Migräne gelitten? & $\square$ JA $\square$ NEIN \\
\hline 11 & $\begin{array}{l}\text { Haben Sie -Metallimplantate im Kopf- und Wirbelsäulebereich (in den } \\
\text { Kopfbereich eingesetztes Metall, z.B. Clips nach Operation eines }\end{array}$ & $\square$ JA $\square$ NEIN \\
\hline
\end{tabular}




\begin{tabular}{|c|c|c|}
\hline & $\begin{array}{l}\text { intrazerebralen Aneurysmas (Gefäßaussackung im Bereich der Gehirngefäße), } \\
\text { Implantation eine künstlichen Hörschnecke) }\end{array}$ & \\
\hline 12 & $\begin{array}{l}\text { Sind Sie Träger eines Herzschrittmachers, Defibrillators, Hörgeräts, } \\
\text { Medikamentenpumpe (Insulin?), Neurostimulators, Implantat mit } \\
\text { Magnetventil (z.B. künstlicher Darmausgang)? Wenn ja, welche? }\end{array}$ & $\square$ JA $\square$ NEIN \\
\hline 13 & Leiden Sie an Schlafstörungen? & $\square$ JA $\square$ NEIN \\
\hline 14 & Haben Sie Herzrhythmusstörungen? & $\square$ JA $\square$ NEIN \\
\hline 15 & Wurden Sie innerhalb der letzten zwei Monate operiert? Wenn ja, woran? & $\square$ JA $\square$ NEIN \\
\hline 16 & $\begin{array}{l}\text { Haben Sie in den letzten } 5 \text { Tagen an einer MRT-, tDCS-, tACS- oder TMS- } \\
\text { Untersuchung teilgenommen? }\end{array}$ & $\square$ JA $\square$ NEIN \\
\hline 17 & $\begin{array}{l}\text { Nehmen Sie zur Zeit gleichzeitig an einem anderem Experiment teil? Wenn } \\
\text { ja, woran? }\end{array}$ & $\square$ JA $\square$ NEIN \\
\hline
\end{tabular}

Nur von Frauen auszufüllen:

\begin{tabular}{|l|l|l|}
\hline 18 & Besteht die Möglichkeit, dass Sie schwanger sind? & $\square$ JA $\square$ NEIN \\
\hline 19 & Stillen Sie im Moment? & $\square$ JA $\square$ NEIN \\
\hline
\end{tabular}

Ich habe die Fragen gelesen und verstanden. Ich erkläre darüber hinaus, dass ich alle Angaben zur Krankengeschichte wahrheitsgemäß gemacht habe.

(Datum, Ort)

(Name, Vorname)

(Unterschrift) 
— wird vom med. Fachpersonal ausgefüllt

— Untersuchung -

Probanden ID:

RR:

Puls:

Körpergröße:

Gewicht:

Medikation (ggf.):

Gang:

Okulomotorik:

Auffälligkeiten (Haut, Sprache, usw):

— Vorerkrankungen —

Epilepsie:

Migräne:

Metallimplantate:

Herzerkrankungen:

Operationen:

Chronische und genetische Krankheiten:

(Datum, Ort)

(Name, Vorname)

(Unterschrift)

Figure A.2: Questionnaire on exclusion criteria for non-invasive brain stimulation and contents of the medical history and physical examination. Abnormities in the anamnesis and examination and affirmed exclusion criteria in the questionnaire led to the rejection of the participation in the study. 


\section{$7 \quad$ References}

Abd Hamid AI, Gall C, Speck O, Antal A, Sabel BA (2015): Effects of alternating current stimulation on the healthy and diseased brain. Front Neurosci $\underline{9}, 391$

Adachi I, Chou DP, Hampton RR (2009): Thatcher effect in monkeys demonstrates conservation of face perception across primates. Curr Biol 19, 1270-1273

Albouy P, Weiss A, Baillet S, Zatorre RJ (2017): Selective entrainment of theta oscillations in the dorsal stream causally enhances auditory working memory performance. Neuron $\underline{94}, 1-14$

Alekseichuk I, Turi Z, Amador de Lara G, Antal A, Paulus W (2016): Spatial working memory in humans depends on theta and high gamma synchronization in the prefrontal cortex. Curr Biol 26, $1513-1521$

Ali MM, Sellers KK, Fröhlich F (2013): Transcranial alternating current stimulation modulates large-scale cortical network activity by network resonance. J Neurosci $\underline{33}, 11262-11275$

Allen TA, Fortin NJ (2013): The evolution of episodic memory. Proc Natl Acad Sci 110, 10379_ 10386

Andersen N, Krauth N, Nabavi S (2017): Hebbian plasticity in vivo: relevance and induction. Curr Opin Neurobiol $\underline{45}, 188-192$

Antal A, Paulus W (2013): Transcranial alternating current stimulation (tACS). Front Hum Neurosci $\underline{7}, 317$

Antal A, Herrmann CS (2016): Transcranial alternating current and random noise stimulation: possible mechanisms. Neural Plast 2016, 1-12

Antal A, Boros K, Poreisz C, Chaieb L, Terney D, Paulus W (2008): Comparatively weak aftereffects of transcranial alternating current stimulation (tACS) on cortical excitability in humans. Brain Stimul 1, 97-105

Antal A, Alekseichuk I, Bikson M, Brockmöller J, Brunoni AR, Chen R, Cohen LG, Dowthwaite G, Ellrich J, Flöel A, et al. (2017): Low intensity transcranial electric stimulation: safety, ethical, legal regulatory and application guidelines. Clin Neurophysiol 128, 1774-1809

Antonenko D, Diekelmann S, Olsen C, Born J, Mölle M (2013): Napping to renew learning capacity: enhanced encoding after stimulation of sleep slow oscillations. Eur J Neurosci $\underline{37}$, 1142-1151

Antonenko D, Faxel M, Grittner U, Lavidor M, Flöel A (2016): Effects of transcranial alternating current stimulation on cognitive functions in healthy young and older adults. Neural Plast 2016, $1-13$

Antony JW, Ferreira CS, Norman KA, Wimber M (2017): Retrieval as a fast route to memory consolidation. Trends Cogn Sci 21, 573-576

Arcurio LR, Gold JM, James TW (2012): The response of face-selective cortex with single face parts and part combinations. Neuropsychologia $\underline{50}, 2454-2459$ 
Atkinson RC, Shiffrin RM: Human memory: A proposed system and its control processes. In: Spence KW, Spence JT (Hrsg.): The psychology of learning and motivation. Academic Press, New York 1968, 89-195

Bailey CH, Kandel ER, Harris KM (2015): Structural components of synaptic plasticity and memory consolidation. Cold Spring Harb Perspect Biol 7, a021758

Barbas H: Frontal cortex. In: Pfaff DW (Hrsg.): Neuroscience in the $21^{\text {st }}$ century. Springer New York, NY, New York 2013, 1289-1334

Baron-Cohen S, Wheelwright S (2004): The empathy quotient: An investigation of adults with asperger syndrome or high functioning autism, and normal sex differences. J Autism Dev Disord 34, 163-175

Belluscio MA, Mizuseki K, Schmidt R, Kempter R, Buzsáki G (2012): Cross-frequency phase-phase coupling between theta and gamma oscillations in the hippocampus. J Neurosci $\underline{32}, 423-435$

Berens SC, Horner AJ (2017): Theta rhythm: temporal glue for episodic memory. Curr Biol 27, R1110-R1112

Bergmann HC, Daselaar SM, Fernández G, Kessels RPC (2016): Neural substrates of successful working memory and long-term memory formation in a relational spatial memory task. Cogn Process 17, 377-387

Bikson M, Grossman P, Thomas C, Zannou AL, Jiang J, Adnan T, Mourdoukoutas AP, Kronberg G, Truong D, Boggio P, et al. (2016): Safety of transcranial direct current stimulation: evidence based update 2016. Brain Stimul 9, 641-661

Bindman LJ, Lippold OC, Redfearn JW (1964): The action of brief polarizing currents on the cerebral cortex of the rat (1) during current flow and (2) in the production of long-lasting after-effects. J Physiol $\underline{172}$, 369-382

Bliss TVP, Cooke SF (2006): Plasticity in the human central nervous system. Brain 129, 1659-1673

Blumenfeld RS, Parks CM, Yonelinas AP, Ranganath C (2011): Putting the pieces together: the role of dorsolateral prefrontal cortex in relational memory encoding. J Cogn Neurosci $\underline{23}$, 257-265

Bonnefond M, Kastner S, Jensen O (2017): Communication between brain areas based on nested oscillations. eNeuro 4, ENEURO.0153-16.2017

Bono J, Wilmes KA, Clopath C (2017): Modelling plasticity in dendrites: from single cells to networks. Curr Opin Neurobiol 뜨, 136-141

Brignani D, Ruzzoli M, Mauri P, Miniussi C (2013): Is transcranial alternating current stimulation effective in modulating brain oscillations? PLoS One $\underline{8}$, e56589

Brown TH, Zhao Y, Leung V: Hebbian Plasticity. In: Squire LR (Hrsg.): Encyclopedia of neuroscience. Academic Press, Oxford 2009, 1049-1056

Brunoni AR, Nitsche MA, Bolognini N, Bikson M, Wagner T, Merabet L, Edwards DJ, ValeroCabre A, Rotenberg A, Pascual-Leone A, et al. (2012): Clinical research with transcranial direct current stimulation (tDCS): challenges and future directions. Brain Stimul $\underline{5}, 175-195$ 
Buzsáki G (1989): Two-stage model of memory trace formation: A role for "noisy" brain states. Neuroscience $\underline{31}, 551-570$

Buzsáki G (2013): Time, space and memory. Nature 497, 568-569

Buzsáki G, Silva FL (2012): High frequency oscillations in the intact brain. Prog Neurobiol $\underline{98}, 241-$ 249

Buzsáki G, Schomburg EW (2015): What does gamma coherence tell us about inter-regional neural communication? Nat Neurosci $\underline{18}, 484-489$

Buzsáki G, Logothetis N, Singer W (2013): Scaling brain size, keeping timing: evolutionary preservation of brain rhythms. Neuron $\underline{80}, 751-764$

Chaudhuri R, Fiete I (2016): Computational principles of memory. Nat Neurosci 19, 394-403

Cirillo G, Di Pino G, Capone F, Ranieri F, Florio L, Todisco V, Tedeschi G, Funke K, Di Lazzaro V (2017): Neurobiological after-effects of non-invasive brain stimulation. Brain Stimul 10, 1 18

Citri A, Malenka RC (2008): Synaptic plasticity: multiple forms, functions and mechanisms. Neuropsychopharmacology $\underline{33}, 18-41$

Clouter A, Shapiro KL, Hanslmayr S (2017): Theta phase synchronization is the glue that binds human associative memory. Curr Biol 글, 3143-3148.e6

Cohen MX (2017): Where does EEG come from and what does it mean? Trends Neurosci $\underline{40}$, 208-218

Cohen N, Squire L (1980): Preserved learning and retention of pattern-analyzing skill in amnesia: dissociation of knowing how and knowing that. Science 210, 207-210

Corrow S, Dalrymple K, Barton J (2016): Prosopagnosia: current perspectives. Eye Brain $\underline{8}, 165$ 175

Cowan N: What are the differences between long-term, short-term, and working memory? Prog Brain Res $\underline{169}, 323-338$

Craik FIM, Lockhart RS (1972): Levels of processing: a framework for memory research. J Verbal Learning Verbal Behav 11, 671-684

De Benedictis A, Duffau H, Paradiso B, Grandi E, Balbi S, Granieri E, Colarusso E, Chioffi F, Marras CE, Sarubbo S (2014): Anatomo-functional study of the temporo-parieto-occipital region: dissection, tractographic and brain mapping evidence from a neurosurgical perspective. J Anat 225, 132-151

Deen B, Koldewyn K, Kanwisher N, Saxe R (2015): Functional organization of social perception and cognition in the superior temporal sulcus. Cereb Cortex $\underline{25}$, 4596-4609

DeGutis JM, Chiu C, Grosso ME, Cohan S (2014): Face processing improvements in prosopagnosia: successes and failures over the last 50 years. Front Hum Neurosci $\underline{8}, 561$

Dragoi G, Buzsáki G (2006): Temporal encoding of place sequences by hippocampal cell assemblies. Neuron $\underline{50}, 145-157$ 
Dudai Y, Karni A, Born J (2015): The consolidation and transformation of memory. Neuron $\underline{88}$, $20-32$

Düzel E, Penny WD, Burgess N (2010): Brain oscillations and memory. Curr Opin Neurobiol 20, 143-149

Eggert T, Dorn H, Sauter C, Nitsche MA, Bajbouj M, Danker-Hopfe H (2013): No effects of slow oscillatory transcranial direct current stimulation (tDCS) on sleep-dependent memory consolidation in healthy elderly subjects. Brain Stimul $\underline{6}$, 938-945

Eichenbaum H (2017): Prefrontal-hippocampal interactions in episodic memory. Nat Rev Neurosci $\underline{18}, 547-558$

Elbich DB, Scherf S (2017): Beyond the FFA: brain-behavior correspondences in face recognition abilities. Neuroimage 147, 409-422

Feldman DE (2012): The spike-timing dependence of plasticity. Neuron $\underline{75}$, 556-571

Fell J, Axmacher N (2011): The role of phase synchronization in memory processes. Nat Rev Neurosci 12, 105-118

Fertonani A, Miniussi C (2017): Transcranial electrical stimulation. Neurosci $\underline{23}$, 109-123

Fertonani A, Ferrari C, Miniussi C (2015): What do you feel if I apply transcranial electric stimulation? Safety, sensations and secondary induced effects. Clin Neurophysiol $\underline{126}, 2181-$ 2188

Fries P (2005): A mechanism for cognitive dynamics: neuronal communication through neuronal coherence. Trends Cogn Sci $\underline{9}, 474-480$

Fröhlich F, Sellers KK, Cordle AL (2015): Targeting the neurophysiology of cognitive systems with transcranial alternating current stimulation. Expert Rev Neurother 15, 145-167

Gainotti G, Marra C (2011): Differential contribution of right and left temporo-occipital and anterior temporal lesions to face recognition disorders. Front Hum Neurosci $\underline{5}, 55$

Ganguly K, Poo M (2013): Activity-dependent neural plasticity from bench to bedside. Neuron $\underline{80}$, 729-741

Gebodh N, Esmaeilpour Z, Adair D, Chelette K, Dmochowski J, Woods AJ, Kappenman ES, Parra LC, Bikson M (2019): Inherent physiological artifacts in EEG during tDCS. Neuroimage $\underline{185}, 408-424$

Geib BR, Stanley ML, Dennis NA, Woldorff MG, Cabeza R (2017): From hippocampus to wholebrain: the role of integrative processing in episodic memory retrieval. Hum Brain Mapp $\underline{38}$, 2242-2259

Geiger MJ, O'Gorman Tuura R, Klaver P (2016): Inter-hemispheric connectivity in the fusiform gyrus supports memory consolidation for faces. Eur J Neurosci $\underline{43}, 1137-1145$

Gildenberg PL (2005): Evolution of neuromodulation. Stereotact Funct Neurosurg $\underline{83}$, 71-79

Girotti M, Adler SM, Bulin SE, Fucich EA, Paredes D, Morilak DA (2018): Prefrontal cortex executive processes affected by stress in health and disease. Prog Neuro-Psychopharmacology Biol Psychiatry $\underline{85}, 161-179$ 
Grüter T, Grüter M, Carbon C-C (2008): Neural and genetic foundations of face recognition and prosopagnosia. J Neuropsychol 2, 79-97

Guderian S, Schott BH, Richardson-Klavehn A, Düzel E (2009): Medial temporal theta state before an event predicts episodic encoding success in humans. Proc Natl Acad Sci 106, 5365-5370

Hanslmayr S, Volberg G, Wimber M, Raabe M, Greenlee MW, Bäuml KH (2011): The relationship between brain oscillations and BOLD signal during memory formation: a combined EEGfMRI study. J Neurosci $\underline{31}, 15674-15680$

Hanslmayr S, Staresina BP, Bowman H (2016): Oscillations and episodic memory :addressing the synchronization/desynchronization conundrum. Trends Neurosci $\underline{39}, 16-25$

Henke K (2010): A model for memory systems based on processing modes rather than consciousness. Nat Rev Neurosci $\underline{11}, 523-532$

Henry MJ, Obleser J (2012): Frequency modulation entrains slow neural oscillations and optimizes human listening behavior. Proc Natl Acad Sci 109, 20095-20100

Herrmann CS, Rach S, Neuling T, Strüber D (2013): Transcranial alternating current stimulation: a review of the underlying mechanisms and modulation of cognitive processes. Front Hum Neurosci $7,1-13$

Herrmann CS, Strüber D, Helfrich RF, Engel AK (2016a): EEG oscillations: from correlation to causality. Int J Psychophysiol $\underline{103}, 12-21$

Herrmann CS, Murray MM, Ionta S, Hutt A, Lefebvre J (2016b): Shaping intrinsic neural oscillations with periodic stimulation. J Neurosci $\underline{36}, 5328-5337$

Hill NJ, Gupta D, Brunner P, Gunduz A, Adamo MA, Ritaccio A, Schalk G (2012): Recording human electrocorticographic $(\mathrm{ECoG})$ signals for neuroscientific research and real-time functional cortical mapping. J Vis $\operatorname{Exp} \underline{64}, 3993$

Hopfinger JB, Parsons J, Fröhlich F (2017): Differential effects of 10-Hz and 40-Hz transcranial alternating current stimulation (tACS) on endogenous versus exogenous attention. Cogn Neurosci $\underline{8}, 102-111$

Ison MJ, Quian Quiroga R, Fried I (2015): Rapid encoding of new memories by individual neurons in the human brain. Neuron $\underline{87}, 220-230$

Jackson AF, Bolger DJ (2014): The neurophysiological bases of EEG and EEG measurement: a review for the rest of us. Psychophysiology $\underline{51,1061-1071}$

Jamil A, Batsikadze G, Kuo HI, Labruna L, Hasan A, Paulus W, Nitsche MA (2017): Systematic evaluation of the impact of stimulation intensity on neuroplastic after-effects induced by transcranial direct current stimulation. J Physiol 595, 1273-1288

Javadi AH, Glen JC, Halkiopoulos S, Schulz M, Spiers HJ (2017): Oscillatory reinstatement enhances declarative memory. J Neurosci $\underline{37}$, 9939-9944

Jensen O, Mazaheri A (2010): Shaping functional architecture by oscillatory alpha activity: gating by inhibition. Front Hum Neurosci $\underline{4}, 1-8$ 
Kadipasaoglu CM, Conner CR, Baboyan VG, Rollo M, Pieters TA, Tandon N (2017): Network dynamics of human face perception. PLoS One 12, e0188834

Kandel ER, Dudai Y, Mayford MR (2014): The molecular and systems biology of memory. Cell 157, $163-186$

Kanwisher N, Yovel G (2006): The fusiform face area: a cortical region specialized for the perception of faces. Philos Trans R Soc B Biol Sci $\underline{361}$, 2109-2128

Kasten FH, Dowsett J, Herrmann CS (2016): Sustained aftereffect of $\alpha$-tACS lasts up to 70 min after stimulation. Front Hum Neurosci $\underline{10}, 245$

Kennerknecht I, Grueter T, Welling B, Wentzek S, Horst J, Edwards S, Grueter M (2006): First report of prevalence of non-syndromic hereditary prosopagnosia (HPA). Am J Med Genet A $\underline{140}, 1617-1622$

Kim H, Cabeza R (2007): Trusting our memories: dissociating the neural correlates of confidence in veridical versus illusory memories. J Neurosci $\underline{27}$, 12190-12197

Kleinert ML, Szymanski C, Müller V (2017): Frequency-unspecific effects of $\theta$-tACS related to a visuospatial working memory task. Front Hum Neurosci 11, 367

Korotkova T, Ponomarenko A, Monaghan CK, Poulter SL, Cacucci F, Wills T, Hasselmo ME, Lever C (2018): Reconciling the different faces of hippocampal theta: The role of theta oscillations in cognitive, emotional and innate behaviors. Neurosci Biobehav Rev $\underline{85}$, 65-80

Korte M, Schmitz D (2016): Cellular and system biology of memory: timing, molecules, and beyond. Physiol Rev $\underline{96}$, 647-693

Kranz F, Ishai A (2006): Face perception is modulated by sexual preference. Curr Biol 16, 63-68

Krause MR, Vieira PG, Csorba BA, Pilly PK, Pack CC (2019): Transcranial alternating current stimulation entrains single-neuron activity in the primate brain. Proc Natl Acad Sci 116, 57475755

Kukushkin NV, Carew TJ (2017): Memory takes time. Neuron 95, 259-279

Kumar S, Zomorrodi R, Ghazala Z, Goodman MS, Blumberger DM, Cheam A, Fischer C, Daskalakis ZJ, Mulsant BH, Pollock BG, Rajji TK (2017): Extent of dorsolateral prefrontal cortex plasticity and its association with working memory in patients with Alzheimer Disease. JAMA Psychiatry $\underline{74}, 1266-1274$

Lage C, Wiles K, Shergill SS, Tracy DK (2016): A systematic review of the effects of low-frequency repetitive transcranial magnetic stimulation on cognition. J Neural Transm $\underline{123}, 1479-1490$

Lefaucheur JP, Antal A, Ayache SS, Benninger DH, Brunelin J, Cogiamanian F, Cotelli M, De Ridder D, Ferrucci R, Langguth B, et al. (2017): Evidence-based guidelines on the therapeutic use of transcranial direct current stimulation (tDCS). Clin Neurophysiol $\underline{128}, 56-92$

Lefebvre S, Liew SL (2017): Anatomical parameters of tDCS to modulate the motor system after stroke: a review. Front Neurol $\underline{8}, 29$

Leopold DA, Rhodes G (2010): A comparative view of face perception. J Comp Psychol 124, 233251 
Li J, Dong M, Ren A, Ren J, Zhang J, Huang L (2016): Structural attributes of the temporal lobe predict face recognition ability in youth. Neuropsychologia $\underline{84}, 1-6$

Limoge A, Robert C, Stanley TH (1999): Transcutaneous cranial electrical stimulation (TCES): a review 1998. Neurosci Biobehav Rev 23, 529-538

Lisman J (2010): Working memory: the importance of theta and gamma oscillations. Curr Biol 20, R490-R492

Lisman JE, Jensen O (2013): The theta-gamma neural code. Neuron 77, 1002-1016

Little AC, Jones BC, DeBruine LM (2011): The many faces of research on face perception. Philos Trans R Soc Lond B Biol Sci $\underline{366}$, 1634-1637

Lopes da Silva F (2013): EEG and MEG: relevance to neuroscience. Neuron 무, 1112-1128

Mahr JB, Csibra G (2018): Why do we remember? The communicative function of episodic memory. Behav Brain Sci 1ㅗ, 1-93

Marin BM, VanHaerents SA, Voss JL, Bridge DJ (2018): Prefrontal $\theta$-burst stimulation disrupts the organizing influence of active short-term retrieval on episodic memory. eNeuro $\underline{5}$, ENEURO.0347-17.2018

Marshall L, Kirov R, Brade J, Mölle M, Born J (2011): Transcranial electrical currents to probe EEG brain rhythms and memory consolidation during sleep in humans. PLoS One $\underline{6}$, e16905

Martinez AM (2017): Computational models of face perception. Curr Dir Psychol Sci 26, 263-269

Miranda PC, Mekonnen A, Salvador R, Ruffini G (2013): The electric field in the cortex during transcranial current stimulation. Neuroimage $\underline{70}, 48-58$

Moran LV., Hong LE (2011): High vs low frequency neural oscillations in schizophrenia. Schizophr Bull $\underline{37}$, 659-663

Morris R: Neurobiology of learning and memory. In: Pfaff DW (Hrsg.): Neuroscience in the $21^{\text {st }}$ century. Springer New York, NY, New York 2013, 2173-2211

Moscovitch M, Cabeza R, Winocur G, Nadel L (2016): Episodic memory and beyond: the hippocampus and neocortex in transformation. Annu Rev Psychol 67, 105-134

Murray JD, Jaramillo J, Wang XJ (2017): Working memory and decision-making in a frontoparietal circuit model. J Neurosci $\underline{37}, 12167-12186$

Naro A, Corallo F, De Salvo S, Marra A, Di Lorenzo G, Muscarà N, Russo M, Marino S, De Luca R, Bramanti P, Calabrò RS (2016): Promising role of neuromodulation in predicting the progression of mild cognitive impairment to dementia. J Alzheimer's Dis $\underline{53}, 1375-1388$

Noury N, Siegel M (2018): Analyzing EEG and MEG signals recorded during tES, a reply. Neuroimage 167, 53-61

Nudo RJ (2013): Recovery after brain injury: mechanisms and principles. Front Hum Neurosci $\underline{7}$, $1-14$

Nyhus E, Curran T (2010): Functional role of gamma and theta oscillations in episodic memory. Neurosci Biobehav Rev 34, 1023-1035 
Osipova D, Takashima A, Oostenveld R, Fernandez G, Maris E, Jensen O (2006): Theta and gamma oscillations predict encoding and retrieval of declarative memory. J Neurosci 26,7523 7531

Ozen S, Sirota A, Belluscio MA, Anastassiou CA, Stark E, Koch C, Buzsáki G (2010): Transcranial electric stimulation entrains cortical neuronal populations in rats. J Neurosci $\underline{30}, 11476-11485$

Pahor A, Jaušovec N (2014): The effects of theta transcranial alternating current stimulation (tACS)

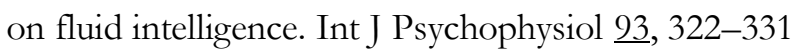

Park Y, Choi W, Paik SB (2017): Symmetry of learning rate in synaptic plasticity modulates formation of flexible and stable memories. Sci Rep $\underline{7}, 5671$

Pascual-Leone A, Amedi A, Fregni F, Merabet LB (2005): The plastic human brain cortex. Annu Rev Neurosci $\underline{28}, 377-401$

Pastötter B, Bäuml KH (2014): Distinct slow and fast cortical theta dynamics in episodic memory retrieval. Neuroimage $\underline{94}, 155-161$

Paulus W (2011): Transcranial electrical stimulation (tES - tDCS; tRNS, tACS) methods. Neuropsychol Rehabil 21, 602-617

Peirce JW (2007): PsychoPy--Psychophysics software in Python. J Neurosci Methods 162, 8-13

Pevzner A, Izadi A, Lee DJ, Shahlaie K, Gurkoff GG (2016): Making waves in the brain: what are oscillations, and why modulating them makes sense for brain injury. Front Syst Neurosci $\underline{10}$, $1-18$

Piccolino M (1998): Animal electricity and the birth of electrophysiology: the legacy of Luigi Galvani. Brain Res Bull 46, 381-407

Pitcher D, Walsh V, Duchaine B (2011): The role of the occipital face area in the cortical face perception network. Exp Brain Res 209, 481-493

Polanía R, Nitsche MA, Korman C, Batsikadze G, Paulus W (2012): The importance of timing in segregated theta phase-coupling for cognitive performance. Curr Biol 22, 1314-1318

Poo MM, Pignatelli M, Ryan TJ, Tonegawa S, Bonhoeffer T, Martin KC, Rudenko A, Tsai LH, Tsien RW, Fishell G, et al. (2016): What is memory? The present state of the engram. BMC Biol 14, 40

Prehn-Kristensen A, Munz M, Göder R, Wilhelm I, Korr K, Vahl W, Wiesner CD, Baving L (2014): Transcranial oscillatory direct current stimulation during sleep improves declarative memory consolidation in children with attention-deficit/hyperactivity disorder to a level comparable to healthy controls. Brain Stimul $\mathbf{7}, 793-799$

Priori A (2003): Brain polarization in humans: a reappraisal of an old tool for prolonged noninvasive modulation of brain excitability. Clin Neurophysiol $114,589-595$

Proverbio AM, Zani A, Adorni R (2008): Neural markers of a greater female responsiveness to social stimuli. BMC Neurosci $\underline{9}, 56$

Proverbio AM (2017): Sex differences in social cognition: The case of face processing. J Neurosci Res $\underline{95}, 222-234$ 
Reinhart RMG, Cosman JD, Fukuda K, Woodman GF (2017): Using transcranial direct-current stimulation (tDCS) to understand cognitive processing. Attention, Perception, Psychophys $\underline{79}$, $3-23$

Rioult-Pedotti MS, Donoghue JP, Dunaevsky A (2007): Plasticity of the synaptic modification range. J Neurophysiol 모, 3688-3695

Robinson JL, Barron DS, Kirby LAJ, Bottenhorn KL, Hill AC, Murphy JE, Katz JS, Salibi N, Eickhoff SB, Fox PT (2015): Neurofunctional topography of the human hippocampus. Hum Brain Mapp 므, 5018-5037

Romanska A, Rezlescu C, Susilo T, Duchaine B, Banissy MJ (2015): High-frequency transcranial random noise stimulation enhances perception of facial identity. Cereb Cortex $\underline{25}, 4334-4340$

Rossi S, Hallett M, Rossini PM, Pascual-Leone A (2009): Safety, ethical considerations, and application guidelines for the use of transcranial magnetic stimulation in clinical practice and research. Clin Neurophysiol 120, 2008-2039

Rossi S, Santarnecchi E, Valenza G, Ulivelli M (2016): The heart side of brain neuromodulation. Philos Trans R Soc A Math Phys Eng Sci 374, 2067

Rugg MD, Vilberg KL (2013): Brain networks underlying episodic memory retrieval. Curr Opin Neurobiol 23, 255-260

Rugo KF, Tamler KN, Woodman GF, Maxcey AM (2017): Recognition-induced forgetting of faces in visual long-term memory. Attention, Perception, Psychophys $\underline{79}, 1878-1885$

Santarnecchi E, Brem AK, Levenbaum E, Thompson T, Kadosh RC, Pascual-Leone A (2015): Enhancing cognition using transcranial electrical stimulation. Curr Opin Behav Sci 4 , 171-178

Santarnecchi E, Muller T, Rossi S, Sarkar A, Polizzotto NR, Rossi A, Cohen Kadosh R (2016): Individual differences and specificity of prefrontal gamma frequency-tACS on fluid intelligence capabilities. Cortex $\underline{75}, 33-43$

Schematic anatomical architecture of the brain (cross section): Fusiform gyrus, https://commons.wikimedia.org/wiki/File:Gray727_fusiform_gyrus.png, last downloaded on 26.07.2020

Schnitzler A, Gross J (2005): Normal and pathological oscillatory communication in the brain. Nat Rev Neurosci $\underline{6}, 285-296$

Schutter DJLG (2014): Syncing your brain: electric currents to enhance cognition. Trends Cogn Sci $\underline{18}, 331-333$

Schutter DJLG, Hortensius R (2011): Brain oscillations and frequency-dependent modulation of cortical excitability. Brain Stimul 4, 97-103

Schwabe L, Nader K, Pruessner JC (2014): Reconsolidation of human memory: brain mechanisms and clinical relevance. Biol Psychiatry $\underline{76}$, 274-280

Squire LR, Zola SM (1996): Structure and function of declarative and nondeclarative memory systems. Proc Natl Acad Sci 93, 13515-13522 
Squire LR, Genzel L, Wixted JT, Morris RGM (2015): Memory consolidation. Cold Spring Harb Perspect Biol 7, a021766

Sreeraj V, Shanbhag V, Nawani H, Shivakumar V, Damodharan D, Bose A, Narayanaswamy J, Venkatasubramanian G (2017): Feasibility of online neuromodulation using transcranial alternating current stimulation in schizophrenia. Indian J Psychol Med $\underline{39}, 92-95$

Tavakoli A V., Yun K (2017): Transcranial alternating current stimulation (tACS) mechanisms and protocols. Front Cell Neurosci 11, 1-10

Tsao DY, Livingstone MS (2008): Mechanisms of face perception. Annu Rev Neurosci $\underline{31}, 411-437$

Tulving E (1986): Episodic and semantic memory: where should we go from here? Behav Brain Sci 9, 573-577

Tulving E (2002): Episodic memory: from mind to brain. Annu Rev Psychol $\underline{53}, 1-25$

Turi Z, Ambrus GG, Janacsek K, Emmert K, Hahn L, Paulus W, Antal A (2013): Both the cutaneous sensation and phosphene perception are modulated in a frequency-specific manner during transcranial alternating current stimulation. Restor Neurol Neurosci 31, 275-285

Vedam-Mai V, Gardner B, Okun MS, Siebzehnrubl FA, Kam M, Aponso P, Steindler DA, Yachnis AT, Neal D, Oliver BU, et al. (2014): Increased precursor cell proliferation after deep brain stimulation for Parkinson's Disease: a human study. PLoS One $\underline{9}$, e88770

Vöröslakos M, Takeuchi Y, Brinyiczki K, Zombori T, Oliva A, Fernández-Ruiz A, Kozák G, Kincses ZT, Iványi B, Buzsáki G, Berényi A (2018): Direct effects of transcranial electric stimulation on brain circuits in rats and humans. Nat Commun $\underline{9}, 483$

Voss JL, Gonsalves BD, Federmeier KD, Tranel D, Cohen NJ (2011): Hippocampal brain-network coordination during volitional exploratory behavior enhances learning. Nat Neurosci $\underline{14}, 115$ 120

Vosskuhl J, Huster RJ, Herrmann CS (2015): Increase in short-term memory capacity induced by down-regulating individual theta frequency via transcranial alternating current stimulation. Front Hum Neurosci $\underline{9}, 257$

Walker MP, Stickgold R (2004): Sleep-dependent learning and memory consolidation. Neuron 44 , 121-133

Wang Y, Wu Y, Sun D, Wang Y, Wang Y (2016): Tracing short connections of the temporoparieto-occipital region in the human brain using diffusion spectrum imaging and fiber dissection. Brain Res 1646, 152-159

Watson TL (2013): Implications of holistic face processing in autism and schizophrenia. Front Psychol 4,414

Weibert K, Andrews TJ (2015): Activity in the right fusiform face area predicts the behavioural advantage for the perception of familiar faces. Neuropsychologia $\underline{75}, 588-596$

Weigelt S, Koldewyn K, Kanwisher N (2012): Face identity recognition in autism spectrum disorders: a review of behavioral studies. Neurosci Biobehav Rev $\underline{36}, 1060-1084$ 
Wilkinson D, Nicholls S, Pattenden C, Kilduff P, Milberg W (2008): Galvanic vestibular stimulation speeds visual memory recall. Exp brain Res 189, 243-248

Wortman-Jutt S, Edwards DJ (2017): Transcranial direct current stimulation in poststroke aphasia recovery. Stroke $\underline{48}, 820-826$

Yavari F, Jamil A, Mosayebi Samani M, Vidor LP, Nitsche MA (2018): Basic and functional effects of transcranial electrical stimulation (tES) - An introduction. Neurosci Biobehav Rev $\underline{85}, 81-$ 92

Zaghi S, Acar M, Hultgren B, Boggio PS, Fregni F (2010): Noninvasive brain stimulation with lowintensity electrical currents: putative mechanisms of action for direct and alternating current stimulation. Neurosci 16, 285-307

Zhao Y, Zhen Z, Liu X, Song Y, Liu J (2018): The neural network for face recognition: insights from an fMRI study on developmental prosopagnosia. Neuroimage 169, 151-161

Ziemann U (2017): Thirty years of transcranial magnetic stimulation: where do we stand? Exp Brain Res $\underline{235}, 973-984$ 


\section{Acknowledgements}

First of all, I want to thank my supervisor Prof. Dr. med. W. Paulus for giving me the opportunity to pursue a doctoral thesis under his mentorship. I also thank him for the laborious work of proofreading and providing me with his scientific advice.

I would also like to thank my second supervisor PD Dr. rer. nat. K. Jordan for providing me her support and valuable advisory opinion.

My personal and warmest thanks go to Dr. Ivan Alekseichuk for his excellent support and his tireless readiness to explain and discuss and to perpetually share his expertise with me. He accompanied me learning theoretical principles, guided me with technical advice for my experiments and provided me invaluable support during the writing process at any time across any distance.

Furthermore, I would like to acknowledge Dr. Zsolt Turi for his great technical assistance during the preparation and conduct of the experiments and for his permanent availability for questions.

At last, I want to thank the whole Department of Clinical Neurophysiology and all volunteers, without whose engagement this work would not have been possible. 